\title{
An existence theorem for Brakke flow with fixed boundary conditions
}

\author{
Salvatore Stuvard ${ }^{1} \cdot$ Yoshihiro Tonegawa $^{2}$
}

Received: 5 December 2019 / Accepted: 30 November 2020 / Published online: 24 January 2021

(c) The Author(s) 2021

\begin{abstract}
Consider an arbitrary closed, countably $n$-rectifiable set in a strictly convex $(n+1)$ dimensional domain, and suppose that the set has finite $n$-dimensional Hausdorff measure and the complement is not connected. Starting from this given set, we show that there exists a non-trivial Brakke flow with fixed boundary data for all times. As $t \uparrow \infty$, the flow sequentially converges to non-trivial solutions of Plateau's problem in the setting of stationary varifolds.
\end{abstract}

Mathematics Subject Classification Primary 53E10 - 49Q20 · 49Q05

\section{Introduction}

A time-parametrized family $\{\Gamma(t)\}_{t \geq 0}$ of $n$-dimensional surfaces in $\mathbb{R}^{n+1}$ (or in an open domain $U \subset \mathbb{R}^{n+1}$ ) is called a mean curvature flow (abbreviated hereafter as MCF) if the velocity of motion of $\Gamma(t)$ is equal to the mean curvature of $\Gamma(t)$ at each point and time. The aim of the present paper is to establish a global-in-time existence theorem for the MCF $\{\Gamma(t)\}_{t \geq 0}$ starting from a given surface $\Gamma_{0}$ while keeping the boundary of $\Gamma(t)$ fixed for all times $t \geq 0$. In particular, we are interested in the case when the initial surface $\Gamma_{0}$ is not smooth. Typical MCF under consideration in this setting may look like a moving network with multiple junctions for $n=1$, or a moving cluster of bubbles for $n=2$, and they may undergo various topological changes as they evolve. Due to the presence of singularities, we work in the framework of the generalized, measure-theoretic notion of MCF introduced by Brakke and since known as the Brakke flow [2,38]. A global-in-time existence result for a Brakke flow without fixed boundary conditions was established by Kim and the second-

Communicated by A. Malchiodi.

$凶$ Yoshihiro Tonegawa

tonegawa@math.titech.ac.jp

Salvatore Stuvard

stuvard@math.utexas.edu

1 Department of Mathematics, The University of Texas at Austin, 2515 Speedway, Stop C1200, Austin, TX 78712-1202, USA

2 Department of Mathematics, Tokyo Institute of Technology, 2-12-1 Ookayama, Meguro-ku, Tokyo 152-8551, Japan 
named author in [20] by reworking [2] thoroughly. The major challenge of the present work is to devise a modification to the approximation scheme in [20] which preserves the boundary data.

Though somewhat technical, in order to clarify the setting of the problem at this point, we state the assumptions on the initial surface $\Gamma_{0}$ and the domain $U$ hosting its evolution. Their validity will be assumed throughout the paper.

Assumption 1.1 Integers $n \geq 1$ and $N \geq 2$ are fixed, and clos $A$ denotes the topological closure of $A$ in $\mathbb{R}^{n+1}$.

(A1) $U \subset \mathbb{R}^{n+1}$ is a strictly convex bounded domain with boundary $\partial U$ of class $C^{2}$.

(A2) $\Gamma_{0} \subset U$ is a relatively closed, countably $n$-rectifiable set with finite $n$-dimensional Hausdorff measure.

(A3) $E_{0,1}, E_{0,2}, \ldots, E_{0, N}$ are non-empty, open, and mutually disjoint subsets of $U$ such that $U \backslash \Gamma_{0}=\bigcup_{i=1}^{N} E_{0, i}$.

(A4) $\partial \Gamma_{0}:=\left(\operatorname{clos} \Gamma_{0}\right) \backslash U$ is not empty, and for each $x \in \partial \Gamma_{0}$ there exist at least two indexes $i_{1} \neq i_{2}$ in $\{1, \ldots, N\}$ such that $x \in \operatorname{clos}\left(\operatorname{clos}\left(E_{0, i_{j}}\right) \backslash\left(U \cup \partial \Gamma_{0}\right)\right)$ for $j=1,2$.

Since $N \geq 2$, we implicitly assume that $U \backslash \Gamma_{0}$ is not connected. When $n=1, \Gamma_{0}$ could be for instance a union of Lipschitz curves joined at junctions, with "labels" from 1 to $N$ being assigned to each connected component of $U \backslash \Gamma_{0}$. If one defines $F_{i}:=\left(\operatorname{clos} E_{0, i}\right) \backslash(U \cup$ $\left.\partial \Gamma_{0}\right)$ for $i=1, \ldots, N$, one can check that each $F_{i}$ is relatively open in $\partial U, F_{1}, \ldots, F_{N}$ are mutually disjoint, and $\cup_{i=1}^{N} F_{i}=\partial U \backslash \partial \Gamma_{0}$. The assumption (A4) is equivalent to the requirement that each $x \in \partial \Gamma_{0}$ is in $\partial F_{i_{1}} \cap \partial F_{i_{2}}$ for some indices $i_{1} \neq i_{2}$. The main result of the present paper can then be roughly stated as follows.

Theorem A Under the assumptions (A1)-(A4), there exists a $M C F\{\Gamma(t)\}_{t \geq 0}$ such that

$$
\Gamma(0)=\Gamma_{0}, \quad \text { and } \quad \partial \Gamma(t):=(\operatorname{clos} \Gamma(t)) \backslash U=\partial \Gamma_{0} \text { for all } t \geq 0 .
$$

For all $t>0, \Gamma(t)$ remains within the convex hull of $\Gamma_{0} \cup \partial \Gamma_{0}$.

More precisely, $\{\Gamma(t)\}_{t \geq 0}$ is a MCF in the sense that $\Gamma(t)$ coincides with the slice, at time $t$, of the space-time support of a Brakke flow $\left\{V_{t}\right\}_{t \geq 0}$ starting from $\Gamma_{0}$. The method adopted to produce the evolving generalized surfaces $\Gamma(t)$ actually gives us more. Indeed, we show the existence of $N$ families $\left\{E_{i}(t)\right\}_{t \geq 0}(i=1, \ldots, N)$ of evolving open sets such that $E_{i}(0)=E_{0, i}$ for every $i$, and $\Gamma(t)=U \backslash \cup_{i=1}^{N} E_{i}(t)$ for all $t \geq 0$. At each time $t \geq 0$, the sets $E_{1}(t), \ldots, E_{N}(t)$ are mutually disjoint and form a partition of $U$. Moreover, for each fixed $i$ the Lebesgue measure of $E_{i}(t)$ is a continuous function of time, so that the evolving $\Gamma(t)$ do not exhibit arbitrary instantaneous loss of mass. See Theorems 2.2 and 2.3 for the full statement.

It is reasonable to expect that the flow $\Gamma(t)$ converges, as $t \rightarrow \infty$, to a minimal surface in $U$ with boundary $\partial \Gamma_{0}$. We are not able to prove such a result in full generality; nonetheless, we can show the following

Theorem B There exists a sequence of times $\left\{t_{k}\right\}_{k=1}^{\infty}$ with $\lim _{k \rightarrow \infty} t_{k}=\infty$ such that the corresponding varifolds $V_{k}:=V_{t_{k}}$ converge to a stationary integral varifold $V_{\infty}$ in $U$ such that $\left(\operatorname{clos}\left(\operatorname{spt}\left\|V_{\infty}\right\|\right)\right) \backslash U=\partial \Gamma_{0}$.

See Corollary 2.4 for a precise statement. The limit $V_{\infty}$ is a solution to Plateau's problem with boundary $\partial \Gamma_{0}$, in the sense that it has the prescribed boundary in the topological sense specified above and it is minimal in the sense of varifolds. We warn the reader that $V_{\infty}$ may not be area-minimizing. Furthermore, the flow may converge to different limit varifolds along 
different diverging sequences of times in all cases when uniqueness of a minimal surface with the prescribed boundary is not guaranteed. The possibility to use Brakke flow in order to select solutions to Plateau's problem in classes of varifolds seems an interesting byproduct of our theory. See Sect. 7 for further discussion on these points.

Next, we discuss closely related results. While there are several works on the global-intime existence of MCF, there are relatively few results on the existence of MCF with fixed boundary conditions. When $\Gamma_{0}$ is a smooth graph over a bounded domain $\Omega$ in $\mathbb{R}^{n}$, globalin-time existence follows from the classical work of Lieberman [25]. Furthermore, under the assumption that $\Omega$ is mean convex, convergence of the flow to the unique solution to the minimal surfaces equation in $\Omega$ with the prescribed boundary was established by Huisken in [16]; see also the subsequent generalizations to the Riemannian setting in [31,34]. The case of network flows with fixed endpoints and a single triple junction was extensively studied in $[28,30]$. For other configurations and related works on the network flows, see the survey paper [29] and references therein. In the case when $N=2$ (which does not allow triple junctions in general), a powerful approach is the level set method $[4,10]$. Existence and uniqueness in this setting were established in [35], and the asymptotic limit as $t \rightarrow \infty$ was studied in [18]. Recently, White [39] proved the existence of a Brakke flow with prescribed smooth boundary in the sense of integral flat chains $\bmod (2)$. The proof uses the elliptic regularization scheme discovered by Ilmanen [17], which allows one to obtain a Brakke flow with additional good regularity and compactness properties; see also [32] for an application of elliptic regularization within the framework of flat chains with coefficients in suitable finite groups to the long-time existence and short-time regularity of unconstrained MCF starting from a general surface cluster. Observe that the homological constraint used by White prevents the flow to develop interior junction-type singularities of odd order (namely, junctions which are locally diffeomorphic to the union of an odd number of half-hyperplanes), because these singularities are necessarily boundary points $\bmod (2)$. As a consequence, the flows obtained in [39] may differ greatly from those produced in the present paper. This is not surprising, as solutions to Brakke flow may be highly non-unique. A complete characterization of the topological changes that the evolving surfaces can undergo with either of the two approaches is, in fact, an interesting open question. It is worth noticing that analogous generic nonuniqueness holds true also for Plateau's problem: in that context, different definitions of the key words surfaces, area, spanning in its formulation lead to solutions with dramatically different regularity properties, thus making each model a better or worse predictor of the geometric complexity of physical soap films; see e.g. the survey papers $[6,15]$ and the references therein, as well as the more recent works [7-9,22-24,27]. It is then interesting and natural to investigate different formulations for Brakke flow as well.

\section{Definitions, notation, and main results}

\subsection{Basic notation}

The ambient space we will be working in is Euclidean space $\mathbb{R}^{n+1}$. We write $\mathbb{R}^{+}$for $[0, \infty)$. For $A \subset \mathbb{R}^{n+1}$, clos $A$ (or $\bar{A}$ ) is the topological closure of $A$ in $\mathbb{R}^{n+1}$ (and not in $U$ ), int $A$ is the set of interior points of $A$ and conv $A$ is the convex hull of $A$. The standard Euclidean inner product between vectors in $\mathbb{R}^{n+1}$ is denoted $x \cdot y$, and $|x|:=\sqrt{x \cdot x}$. If $L, S \in \mathscr{L}\left(\mathbb{R}^{n+1} ; \mathbb{R}^{n+1}\right)$ are linear operators in $\mathbb{R}^{n+1}$, their (Hilbert-Schmidt) inner product is $L \cdot S:=\operatorname{trace}\left(L^{T} \circ S\right)$, where $L^{T}$ is the transpose of $L$ and $\circ$ denotes composition. 
The corresponding (Euclidean) norm in $\mathscr{L}\left(\mathbb{R}^{n+1} ; \mathbb{R}^{n+1}\right)$ is then $|L|:=\sqrt{L \cdot L}$, whereas the operator norm in $\mathscr{L}\left(\mathbb{R}^{n+1} ; \mathbb{R}^{n+1}\right)$ is $\|L\|:=\sup \left\{|L(x)|: x \in \mathbb{R}^{n+1}\right.$ with $\left.|x| \leq 1\right\}$. If $u, v \in \mathbb{R}^{n+1}$ then $u \otimes v \in \mathscr{L}\left(\mathbb{R}^{n+1} ; \mathbb{R}^{n+1}\right)$ is defined by $(u \otimes v)(x):=(x \cdot v) u$, so that $\|u \otimes v\|=|u||v|$. The symbol $U_{r}(x)$ (resp. $\left.B_{r}(x)\right)$ denotes the open (resp. closed) ball in $\mathbb{R}^{n+1}$ centered at $x$ and having radius $r>0$. The Lebesgue measure of a set $A \subset \mathbb{R}^{n+1}$ is denoted $\mathcal{L}^{n+1}(A)$ or $|A|$. If $1 \leq k \leq n+1$ is an integer, $U_{r}^{k}(x)$ denotes the open ball with center $x$ and radius $r$ in $\mathbb{R}^{k}$. We will set $\omega_{k}:=\mathcal{L}^{k}\left(U_{1}^{k}(0)\right)$. The symbol $\mathcal{H}^{k}$ denotes the $k$-dimensional Hausdorff measure in $\mathbb{R}^{n+1}$, so that $\mathcal{H}^{n+1}$ and $\mathcal{L}^{n+1}$ coincide as measures.

A Radon measure $\mu$ in $U \subset \mathbb{R}^{n+1}$ is always also regarded as a linear functional on the space $C_{c}(U)$ of continuous and compactly supported functions on $U$, with the pairing denoted $\mu(\phi)$ for $\phi \in C_{c}(U)$. The restriction of $\mu$ to a Borel set $A$ is denoted $\mu L_{A}$, so that $\left(\mu\left\llcorner_{A}\right)(E):=\mu(A \cap E)\right.$ for any $E \subset U$. The support of $\mu$ is denoted spt $\mu$, and it is the relatively closed subset of $U$ defined by

$$
\text { spt } \mu:=\left\{x \in U: \mu\left(B_{r}(x)\right)>0 \text { for every } r>0\right\} .
$$

The upper and lower $k$-dimensional densities of a Radon measure $\mu$ at $x \in U$ are

$$
\theta^{* k}(\mu, x):=\limsup _{r \rightarrow 0^{+}} \frac{\mu\left(B_{r}(x)\right)}{\omega_{k} r^{k}}, \quad \theta_{*}^{k}(\mu, x):=\liminf _{r \rightarrow 0^{+}} \frac{\mu\left(B_{r}(x)\right)}{\omega_{k} r^{k}},
$$

respectively. If $\theta^{* k}(\mu, x)=\theta_{*}^{k}(\mu, x)$ then the common value is denoted $\theta^{k}(\mu, x)$, and is called the $k$-dimensional density of $\mu$ at $x$. For $1 \leq p \leq \infty$, the space of $p$-integrable (resp. locally $p$-integrable) functions with respect to $\mu$ is denoted $L^{p}(\mu)$ (resp. $L_{l o c}^{p}(\mu)$ ). For a set $E \subset U, \chi_{E}$ is the characteristic function of $E$. If $E$ is a set of finite perimeter in $U$, then $\nabla \chi_{E}$ is the associated Gauss-Green measure in $U$, and its total variation $\left\|\nabla \chi_{E}\right\|$ in $U$ is the perimeter measure; by De Giorgi's structure theorem, $\left\|\nabla \chi_{E}\right\|=\mathcal{H}^{n}\left\llcorner_{\partial^{*} E}\right.$, where $\partial^{*} E$ is the reduced boundary of $E$ in $U$.

\subsection{Varifolds}

The symbol $\mathbf{G}(n+1, k)$ will denote the Grassmannian of (unoriented) $k$-dimensional linear planes in $\mathbb{R}^{n+1}$. Given $S \in \mathbf{G}(n+1, k)$, we shall often identify $S$ with the orthogonal projection operator onto it. The symbol $\mathbf{V}_{k}(U)$ will denote the space of $k$-dimensional varifolds in $U$, namely the space of Radon measures on $\mathbf{G}_{k}(U):=U \times \mathbf{G}(n+1, k)$ (see [1,33] for a comprehensive treatment of varifolds). To any given $V \in \mathbf{V}_{k}(U)$ one associates a Radon measure $\|V\|$ on $U$, called the weight of $V$, and defined by projecting $V$ onto the first factor in $\mathbf{G}_{k}(U)$, explicitly:

$$
\|V\|(\phi):=\int_{\mathbf{G}_{k}(U)} \phi(x) d V(x, S) \quad \text { for every } \phi \in C_{c}(U) .
$$

A set $\Gamma \subset \mathbb{R}^{n+1}$ is countably $k$-rectifiable if it can be covered by countably many Lipschitz images of $\mathbb{R}^{k}$ into $\mathbb{R}^{n+1}$ up to a $\mathcal{H}^{k}$-negligible set. We say that $\Gamma$ is (locally) $\mathcal{H}^{k}$-rectifiable if it is $\mathcal{H}^{k}$-measurable, countably $k$-rectifiable, and $\mathcal{H}^{k}(\Gamma)$ is (locally) finite. If $\Gamma \subset U$ is locally $\mathcal{H}^{k}$-rectifiable, and $\theta \in L_{l o c}^{1}\left(\mathcal{H}^{k}\left\llcorner_{\Gamma}\right)\right.$ is a positive function on $\Gamma$, then there is a $k$-varifold canonically associated to the pair $(\Gamma, \theta)$, namely the varifold $\operatorname{var}(\Gamma, \theta)$ defined by

$$
\operatorname{var}(\Gamma, \theta)(\varphi):=\int_{\Gamma} \varphi\left(x, T_{x} \Gamma\right) \theta(x) d \mathcal{H}^{k}(x) \quad \text { for every } \varphi \in C_{c}\left(\mathbf{G}_{k}(U)\right)
$$


where $T_{x} \Gamma$ denotes the approximate tangent plane to $\Gamma$ at $x$, which exists $\mathcal{H}^{k}$-a.e. on $\Gamma$. Any varifold $V \in \mathbf{V}_{k}(U)$ admitting a representation as in (2.1) is said to be rectifiable, and the space of rectifiable $k$-varifolds in $U$ is denoted by $\mathbf{R V}_{k}(U)$. If $V=\operatorname{var}(\Gamma, \theta)$ is rectifiable and $\theta(x)$ is an integer at $\mathcal{H}^{k}$-a.e. $x \in \Gamma$, then we say that $V$ is an integral $k$-dimensional varifold in $U$ : the corresponding space is denoted $\mathbf{I} \mathbf{V}_{k}(U)$.

\subsection{First variation of a varifold}

If $V \in \mathbf{V}_{k}(U)$ and $f: U \rightarrow U^{\prime}$ is $C^{1}$ and proper, then we let $f_{\sharp} V \in \mathbf{V}_{k}\left(U^{\prime}\right)$ denote the push-forward of $V$ through $f$. Recall that the weight of $f_{\sharp} V$ is given by

$$
\left\|f_{\sharp} V\right\|(\phi)=\int_{\mathbf{G}_{k}(U)} \phi \circ f(x)\left|\Lambda_{k} \nabla f(x) \circ S\right| d V(x, S) \quad \text { for every } \phi \in C_{c}\left(U^{\prime}\right),
$$

where

$$
\begin{aligned}
& \left|\Lambda_{k} \nabla f(x) \circ S\right|:=\left|\nabla f(x) \cdot v_{1} \wedge \ldots \wedge \nabla f(x) \cdot v_{k}\right| \\
& \quad \text { for any orthonormal basis }\left\{v_{1}, \ldots, v_{k}\right\} \text { of } S
\end{aligned}
$$

is the Jacobian of $f$ along $S \in \mathbf{G}(n+1, k)$. Given a varifold $V \in \mathbf{V}_{k}(U)$ and a vector field $g \in C_{c}^{1}\left(U ; \mathbb{R}^{n+1}\right)$, the first variation of $V$ in the direction of $g$ is the quantity

$$
\delta V(g):=\left.\frac{d}{d t}\right|_{t=0}\left\|\left(\Phi_{t}\right)_{\sharp} V\right\|(\tilde{U}),
$$

where $\Phi_{t}(\cdot)=\Phi(t, \cdot)$ is any one-parameter family of diffeomorphisms of $U$ defined for sufficiently small $|t|$ such that $\Phi_{0}=\mathrm{id}_{U}$ and $\partial_{t} \Phi(0, \cdot)=g(\cdot)$. The $\tilde{U}$ is chosen so that clos $\tilde{U} \subset U$ is compact and spt $g \subset \tilde{U}$, and the definition of (2.3) does not depend on the choice of $\tilde{U}$. It is well known that $\delta V$ is a linear and continuous functional on $C_{c}^{1}\left(U ; \mathbb{R}^{n+1}\right)$, and in fact that

$$
\delta V(g)=\int_{\mathbf{G}_{k}(U)} \nabla g(x) \cdot S d V(x, S) \quad \text { for every } g \in C_{c}^{1}\left(U ; \mathbb{R}^{n+1}\right),
$$

where, after identifying $S \in \mathbf{G}(n+1, k)$ with the orthogonal projection operator $\mathbb{R}^{n+1} \rightarrow S$,

$$
\nabla g \cdot S=\operatorname{trace}\left(\nabla g^{T} \circ S\right)=\sum_{i, j=1}^{n+1} S_{i j} \frac{\partial g_{i}}{\partial x_{j}}=\operatorname{div}^{S} g
$$

If $\delta V$ can be extended to a linear and continuous functional on $C_{c}\left(U ; \mathbb{R}^{n+1}\right)$, we say that $V$ has bounded first variation in $U$. In this case, $\delta V$ is naturally associated with a unique $\mathbb{R}^{n+1}$-valued measure on $U$ by means of the Riesz representation theorem. If such a measure is absolutely continuous with respect to the weight $\|V\|$, then there exists a $\|V\|$-measurable and locally $\|V\|$-integrable vector field $h(\cdot, V)$ such that

$$
\delta V(g)=-\int_{U} g(x) \cdot h(x, V) d\|V\|(x) \quad \text { for every } g \in C_{c}\left(U, \mathbb{R}^{n+1}\right)
$$

by the Lebesgue-Radon-Nikodým differentiation theorem. The vector field $h(\cdot, V)$ is called the generalized mean curvature vector of $V$. In particular, if $\delta V(g)=0$ for all $g \in$ $C_{c}^{1}\left(U ; \mathbb{R}^{n+1}\right), V$ is called stationary, and this is equivalent to $h(\cdot, V)=0\|V\|$-almost 
everywhere. For any $V \in \mathbf{I V}_{k}(U)$ with bounded first variation, Brakke's perpendicularity theorem [2, Chapter 5] says that

$$
S^{\perp}(h(x, V))=h(x, V) \quad \text { for } V \text {-a.e. }(x, S) \in \mathbf{G}_{k}(U) .
$$

Here, $S^{\perp}$ is the projection onto the orthogonal complement of $S$ in $\mathbb{R}^{n+1}$. This means that the generalized mean curvature vector is perpendicular to the approximate tangent plane almost everywhere.

Other than the first variation $\delta V$ discussed above, we shall also use a weighted first variation, defined as follows. For given $\phi \in C_{c}^{1}\left(U ; \mathbb{R}^{+}\right), V \in \mathbf{V}_{k}(U)$, and $g \in C_{c}^{1}\left(U ; \mathbb{R}^{n+1}\right)$, we modify (2.3) to introduce the $\phi$-weighted first variation of $V$ in the direction of $g$, denoted $\delta(V, \phi)(g)$, by setting

$$
\delta(V, \phi)(g):=\left.\frac{d}{d t}\right|_{t=0}\left\|\left(\Phi_{t}\right)_{\sharp} V\right\|(\phi),
$$

where $\Phi_{t}$ denotes the one-parameter family of diffeomorphisms of $U$ induced by $g$ as above. Proceeding as in the derivation of (2.4), one then obtains the expression

$$
\delta(V, \phi)(g)=\int_{\mathbf{G}_{k}(U)} \phi(x) \nabla g(x) \cdot S d V(x, S)+\int_{U} g(x) \cdot \nabla \phi(x) d\|V\|(x) .
$$

Using $\phi \nabla g=\nabla(\phi g)-g \otimes \nabla \phi$ in (2.8) and (2.4), we obtain

$$
\begin{aligned}
\delta(V, \phi)(g) & =\delta V(\phi g)+\int_{\mathbf{G}_{k}(U)} g(x) \cdot(\nabla \phi(x)-S(\nabla \phi(x))) d V(x, S) \\
& =\delta V(\phi g)+\int_{\mathbf{G}_{k}(U)} g(x) \cdot S^{\perp}(\nabla \phi(x)) d V(x, S) .
\end{aligned}
$$

If $\delta V$ has generalized mean curvature $h(\cdot, V)$, then we may use (2.5) in (2.9) to obtain

$$
\delta(V, \phi)(g)=-\int_{U} \phi(x) g(x) \cdot h(x, V) d\|V\|(x)+\int_{\mathbf{G}_{k}(U)} g(x) \cdot S^{\perp}(\nabla \phi(x)) d V(x, S) .
$$

The definition of Brakke flow requires considering weighted first variations in the direction of the mean curvature. Suppose $V \in \mathbf{I V}_{k}(U), \delta V$ is locally bounded and absolutely continuous with respect to $\|V\|$ and $h(\cdot, V)$ is locally square-integrable with respect to $\|V\|$. In this case, it is natural from the expression (2.10) to define for $\phi \in C_{c}^{1}\left(U ; \mathbb{R}^{+}\right)$

$$
\delta(V, \phi)(h(\cdot, V)):=\int_{U}\left\{-\phi(x)|h(x, V)|^{2}+h(x, V) \cdot \nabla \phi(x)\right\} d\|V\|(x) .
$$

Observe that here we have used (2.6) in order to replace the term $h(x, V) \cdot S^{\perp}(\nabla \phi(x))$ with $h(x, V) \cdot \nabla \phi(x)$.

\subsection{Brakke flow}

To motivate a weak formulation of the MCF, note that a smooth family of $k$-dimensional surfaces $\{\Gamma(t)\}_{t \geq 0}$ in $U$ is a MCF if and only if the following inequality holds true for all $\phi=\phi(x, t) \in C_{c}^{1}\left(U \times[0, \infty) ; \mathbb{R}^{+}\right)$:

$$
\frac{d}{d t} \int_{\Gamma(t)} \phi d \mathcal{H}^{k} \leq \int_{\Gamma(t)}\left\{-\phi|h(\cdot, \Gamma(t))|^{2}+\nabla \phi \cdot h(\cdot, \Gamma(t))+\frac{\partial \phi}{\partial t}\right\} d \mathcal{H}^{k}
$$


In fact, the "only if" part holds with equality in place of inequality. For a more comprehensive treatment of the Brakke flow, see [38, Chapter 2]. Formally, if $\partial \Gamma(t) \subset \partial U$ is fixed in time, with $\phi=1$, we also obtain

$$
\frac{d}{d t} \mathcal{H}^{k}(\Gamma(t)) \leq-\int_{\Gamma(t)}|h(x, \Gamma(t))|^{2} d \mathcal{H}^{k}(x),
$$

which states the well-known fact that the $L^{2}$-norm of the mean curvature represents the dissipation of area along the MCF. Motivated by (2.12) and (2.13), and for the purposes of this paper, we give the following definition.

Definition 2.1 We say that a family of varifolds $\left\{V_{t}\right\}_{t \geq 0}$ in $U$ is a Brakke flow with fixed boundary $\Sigma \subset \partial U$ if all of the following hold:

(a) For a.e. $t \geq 0, V_{t} \in \mathbf{I V}_{k}(U)$;

(b) For a.e. $t \geq 0, \delta V_{t}$ is bounded and absolutely continuous with respect to $\left\|V_{t}\right\|$;

(c) The generalized mean curvature $h\left(x, V_{t}\right)$ (which exists for a.e. $t$ by (b)) satisfies for all $T>0$

$$
\left\|V_{T}\right\|(U)+\int_{0}^{T} d t \int_{U}\left|h\left(x, V_{t}\right)\right|^{2} d\left\|V_{t}\right\|(x) \leq\left\|V_{0}\right\|(U) ;
$$

(d) For all $0 \leq t_{1}<t_{2}<\infty$ and $\phi \in C_{c}^{1}\left(U \times \mathbb{R}^{+}\right.$; $\left.\mathbb{R}^{+}\right)$,

$$
\left.\left\|V_{t}\right\|(\phi(\cdot, t))\right|_{t=t_{1}} ^{t_{2}} \leq \int_{t_{1}}^{t_{2}} \delta\left(V_{t}, \phi(\cdot, t)\right)\left(h\left(\cdot, V_{t}\right)\right)+\left\|V_{t}\right\|\left(\frac{\partial \phi}{\partial t}(\cdot, t)\right) d t,
$$

having set $\left.\left\|V_{t}\right\|(\phi(\cdot, t))\right|_{t=t_{1}} ^{t_{2}}:=\left\|V_{t_{2}}\right\|\left(\phi\left(\cdot, t_{2}\right)\right)-\left\|V_{t_{1}}\right\|\left(\phi\left(\cdot, t_{1}\right)\right)$;

(e) For all $t \geq 0$, (clos $\left.\left(\operatorname{spt}\left\|V_{t}\right\|\right)\right) \backslash U=\Sigma$.

In this paper, we are interested in the $n$-dimensional Brakke flow in particular. Formally, by integrating (2.13) from 0 to $T$, we obtain the analogue of (2.14). By integrating (2.12) from $t_{1}$ to $t_{2}$, we also obtain the analogue of (2.15) via the expression (2.11). We recall that the closure is taken with respect to the topology of $\mathbb{R}^{n+1}$ while the support of $\left\|V_{t}\right\|$ is in $U$. Thus (e) geometrically means that "the boundary of $V_{t}$ (or $\left.\left\|V_{t}\right\|\right)$ is $\Sigma$ ".

\subsection{Main results}

The main existence theorem of a Brakke flow with fixed boundary is the following.

Theorem 2.2 Suppose that $U, \Gamma_{0}$, and $E_{0,1}, \ldots, E_{0, N}$ satisfy Assumption 1.1 (A1)-(A4). Then, there exists a Brakke flow $\left\{V_{t}\right\}_{t \geq 0}$ with fixed boundary $\partial \Gamma_{0}$, and $\left\|V_{0}\right\|=\mathcal{H}^{n}\left\llcorner_{\Gamma_{0}}\right.$. If $\mathcal{H}^{n}\left(\Gamma_{0} \backslash \cup_{i=1}^{N} \partial^{*} E_{0, i}\right)=0$, we have $\lim _{t \downarrow 0}\left\|V_{t}\right\|=\mathcal{H}^{n}\left\llcorner_{\Gamma_{0}}\right.$.

Since we are assuming that $\partial \Gamma_{0} \neq \emptyset$, we have $V_{t} \neq 0$ for all $t>0$. If the union of the reduced boundaries of the initial partition in $U$ coincides with $\Gamma_{0}$ modulo $\mathcal{H}^{n}$-negligible sets (note that the assumptions (A2) and (A3) in Assumption 1.1 imply that $\left.\Gamma_{0}=U \cap \bigcup_{i=1}^{N} \partial E_{0, i}\right)$, then the claim is that the initial condition is satisfied continuously as measures. Otherwise, an instantaneous loss of measure may occur at $t=0$. As far as the regularity is concerned, under the additional assumption that $\left\{V_{t}\right\}_{t>0}$ is a unit density flow, partial regularity theorems of $[2,19,37]$ show that $V_{t}$ is a smooth MCF for a.e. time and a.e. point in space, just like [20], see [20, Theorem 3.6] for the precise statement. No claim of the uniqueness is made here, but the next Theorem 2.3 gives an additional structure to $V_{t}$ in the form of "moving partitions" starting from $E_{0,1}, \ldots, E_{0, N}$. 
Theorem 2.3 Under the same assumption of Theorem 2.2 and in addition to $\left\{V_{t}\right\}_{t \geq 0}$, for each $i=1, \ldots, N$ there exists a one-parameter family $\left\{E_{i}(t)\right\}_{t \geq 0}$ of open sets $E_{i}(t) \subset U$ with the following properties. Let $\Gamma(t):=U \backslash \cup_{i=1}^{N} E_{i}(t)$.

(1) $E_{i}(0)=E_{0, i} \forall i=1, \ldots, N$;

(2) $\forall t \geq 0$, the sets $\left\{E_{i}(t)\right\}_{i=1}^{N}$ are mutually disjoint;

(3) $\forall \tilde{U} \subset \subset U$ and $\forall t \geq 0, \mathcal{H}^{n}(\Gamma(t) \cap \tilde{U})<\infty$;

(4) $\forall t \geq 0, \Gamma(t)=U \cap \cup_{i=1}^{N} \partial\left(E_{i}(t)\right)$;

(5) $\forall t \geq 0, \Gamma(t) \subset \operatorname{conv}\left(\Gamma_{0} \cup \partial \Gamma_{0}\right)$;

(6) $\forall t \geq 0$ and $\forall i=1, \ldots, N, E_{i}(t) \backslash \operatorname{conv}\left(\Gamma_{0} \cup \partial \Gamma_{0}\right)=E_{0, i} \backslash \operatorname{conv}\left(\Gamma_{0} \cup \partial \Gamma_{0}\right)$;

(7) $\forall t \geq 0, \partial \Gamma(t):=(\operatorname{clos} \Gamma(t)) \backslash U=\partial \Gamma_{0}$;

(8) $\forall t \geq 0$ and $\forall i=1, \ldots, N,\left\|\nabla \chi_{E_{i}(t)}\right\| \leq\left\|V_{t}\right\|$ and $\sum_{i=1}^{N}\left\|\nabla \chi_{E_{i}(t)}\right\| \leq 2\left\|V_{t}\right\|$;

(9) Fix $i=1, \ldots, N$ and $U_{r}(x) \subset \subset U$, and define $g(t):=\mathcal{L}^{n+1}\left(U_{r}(x) \cap E_{i}(t)\right)$. Then, $g \in C^{0}([0, \infty)) \cap C^{0, \frac{1}{2}}((0, \infty))$

(10) For each $i=1, \ldots, N, \chi_{E_{i}(t)} \in C\left([0, \infty) ; L^{1}(U)\right)$;

(11) Let $\mu$ be the product measure of $\left\|V_{t}\right\|$ and dt defined on $U \times \mathbb{R}^{+}$, i.e. $d \mu:=d\left\|V_{t}\right\| d t$. Then, $\forall t>0$, we have

$$
\text { spt }\left\|V_{t}\right\| \subset\{x \in U:(x, t) \in \operatorname{spt} \mu\}=\Gamma(t) .
$$

The claims (1)-(4) imply that $\left\{E_{i}(t)\right\}_{i=1}^{N}$ is an $\mathcal{L}^{n+1}$-partition of $U$, and that $\Gamma(t)$ has empty interior in particular. The claim (5) is an expected property for the MCF, and, by (11), spt $\left\|V_{t}\right\|$ is also in the same convex hull. (7) says that $\Gamma(t)$ has the fixed boundary $\partial \Gamma_{0}$. In general, the reduced boundary of the partition and $\left\|V_{t}\right\|$ may not match, but the latter is bounded from below by the former as in (8). By (10), the Lebesgue measure of each $E_{i}(t)$ changes continuously in time, so that arbitrary sudden loss of measure of $\left\|V_{t}\right\|$ is not allowed. The statement in (11) says that the time-slice of the support of $\mu$ at time $t$ contains the support of $\left\|V_{t}\right\|$ and is equal to the topological boundary of the moving partition.

As a corollary of the above, we deduce the following.

Corollary 2.4 There exist a sequence $\left\{t_{k}\right\}_{k=1}^{\infty}$ with $\lim _{k \rightarrow \infty} t_{k}=\infty$ and a varifold $V \in$ $\mathbf{I V}_{n}(U)$ such that $V_{t_{k}} \rightarrow V$ in the sense of varifolds. The varifold $V$ is stationary. Furthermore, there is a mutually disjoint family $\left\{E_{i}\right\}_{i=1}^{N}$ of open subsets of $U$ such that

(1) $\forall i=1, \ldots, N,\left\|\nabla \chi_{E_{i}}\right\| \leq\|V\|$ and $\sum_{i=1}^{N}\left\|\nabla \chi_{E_{i}}\right\| \leq 2\|V\|$;

(2) $\forall i=1, \ldots, N, E_{i} \backslash \operatorname{conv}\left(0_{0} \cup @ 0_{0}\right)=E_{0, i} \backslash \operatorname{conv}\left(\Gamma_{0} \cup \partial \Gamma_{0}\right)$;

(3) $U \backslash \bigcup_{i=1}^{N} E_{i}=\operatorname{spt}\|V\|$, and $0<\mathcal{H}^{n}\left(U \backslash \bigcup_{i=1}^{N} E_{i}\right) \leq\|V\|(U) \leq \mathcal{H}^{n}\left(\Gamma_{0}\right)$;

(4) $(\operatorname{clos}(\operatorname{spt}\|V\|)) \backslash U=\left(\operatorname{clos}\left(U \backslash \bigcup_{i=1}^{N} E_{i}\right)\right) \backslash U=\partial \Gamma_{0}$.

The varifold $V$ in Corollary 2.4 is a solution to Plateau's problem in $U$ in the class of stationary varifolds satisfying the topological constraint $(\operatorname{clos}(\operatorname{spt}\|V\|)) \backslash U=\partial \Gamma_{0}$. This is an interesting byproduct of our construction, above all considering that $\partial \Gamma_{0}$ enjoys in general rather poor regularity (in particular, it may have infinite $(n-1)$-dimensional Hausdorff measure, and also it may not be countably $(n-1)$-rectifiable). Even though the topological boundary condition specified above seems natural in this setting, other notions of spanning may be adopted: for instance, in Proposition 7.4 we show that a strong homotopic spanning condition in the sense of $[7,14]$ is preserved along the flow and in the limit if it is satisfied at the initial time $t=0$. We postpone further discussion and questions concerning the application to Plateau's problem to Sect. 7. 


\subsection{General strategy and structure of the paper}

The general idea behind the proof of Theorems 2.2 and 2.3 is to suitably modify the time-discrete approximation scheme introduced in $[2,20]$. There, one constructs a timeparametrized flow of open partitions which is piecewise constant in time. We will call epoch any time interval during which the approximating flow is constant. The open partition at a given epoch is constructed from the open partition at the previous epoch by applying two operations, which we call steps. The first step is a small Lipschitz deformation of partitions with the effect of "regularizing singularities" by "locally minimizing the area of the boundary of partitions" at a small scale. This deformation is defined in such a way that, if the boundary of partitions is regular (relative to a certain length scale), then the deformation reduces to the identity. The second step consists of flowing the boundary of partitions by a suitably defined "approximate mean curvature vector". The latter is computed by smoothing the surface measures via convolution with a localized heat kernel. Note that, typically, the boundary of open partitions has bounded $n$-dimensional measure, but the unit-density varifold associated to it may not have bounded first variation. In [20], a time-discrete approximate MCF is obtained by alternating these two steps, epoch after epoch. In the present work, we need to fix the boundary $\partial \Gamma_{0}$. The rough idea to achieve this is to perform an "exponentially small" truncation of the approximate mean curvature vector near $\partial \Gamma_{0}$, so that the boundary cannot move in the "polynomial time scale" defining an epoch with respect to a certain length scale. We also need to make sure that the time-discrete movement does not push the boundary of open partitions to the outside of $U$. To prevent this, in addition to the two steps (Lipschitz deformation and motion by smoothed and truncated mean curvature vector), we add another "retraction to $U$ " step to be performed in each epoch. All these operations have to come with suitable estimates on the surface measures, in order to have convergence of the approximating flow when we let the epoch time scale approach zero. The final goal is to show that this limit flow is indeed a Brakke flow with fixed boundary $\partial \Gamma_{0}$ as in Definition 2.1.

The rest of the paper is organized as follows. Section 3 lays the foundations to the technical construction of the approximate flow by proving the relevant estimates to be used in the Lipschitz deformation and flow by smoothed mean curvature steps, and by defining the boundary truncation of the mean curvature. Both the discrete approximate flow and its "vanishing epoch" limit are constructed in Sect. 4. In Sect. 5 we show that the one-parameter family of measures obtained in the previous section satisfies conditions (a) to (d) in Definition 2.1. The boundary condition (e) is, instead, proved in Sect. 6, which therefore also contains the proofs of Theorems 2.2 and 2.3. Finally, Sect. 7 is dedicated to the limit $t \rightarrow \infty$ : hence, it contains the proof of Corollary 2.4, as well as a discussion of related results and open questions concerning the application of our construction to Plateau's problem.

\section{Preliminaries}

In this section we will collect the preliminary results that will play a pivotal role in the construction of the time-discrete approximate flows. Some of the results are straightforward adaptations of the corresponding ones in [20]: when that is the case, we shall omit the proofs, and refer the reader to that paper. 


\subsection{Classes of test functions and vector fields}

Define, for every $j \in \mathbb{N}$, the classes $\mathcal{A}_{j}$ and $\mathcal{B}_{j}$ as follows:

$$
\begin{gathered}
\mathcal{A}_{j}:=\left\{\phi \in C^{2}\left(\mathbb{R}^{n+1} ; \mathbb{R}^{+}\right): \phi(x) \leq 1,|\nabla \phi(x)| \leq j \phi(x),\right. \\
\left.\left\|\nabla^{2} \phi(x)\right\| \leq j \phi(x) \text { for every } x \in \mathbb{R}^{n+1}\right\}, \\
\mathcal{B}_{j}:=\left\{g \in C^{2}\left(\mathbb{R}^{n+1} ; \mathbb{R}^{n+1}\right):|g(x)| \leq j,\|\nabla g(x)\| \leq j,\right. \\
\left.\left\|\nabla^{2} g(x)\right\| \leq j \text { for every } x \in \mathbb{R}^{n+1} \text { and }\|g\|_{L^{2}} \leq j\right\} .
\end{gathered}
$$

The properties of functions $\phi \in \mathcal{A}_{j}$ and vector fields $g \in \mathcal{B}_{j}$ are precisely as in [20, Lemma 4.6, Lemma 4.7], and we record them in the following lemma for future reference.

Lemma 3.1 Let $x, y \in \mathbb{R}^{n+1}$ and $j \in \mathbb{N}$. For every $\phi \in \mathcal{A}_{j}$, the following properties hold:

$$
\begin{aligned}
\phi(x) & \leq \phi(y) \exp (j|x-y|), \\
|\phi(x)-\phi(y)| & \leq j|x-y| \phi(y) \exp (j|x-y|), \\
|\phi(x)-\phi(y)-\nabla \phi(y) \cdot(x-y)| & \leq j|x-y|^{2} \phi(y) \exp (j|x-y|) .
\end{aligned}
$$

Also, for every $g \in \mathcal{B}_{j}$ :

$$
|g(x)-g(y)| \leq j|x-y| .
$$

\subsection{Open partitions and admissible functions}

Let $\tilde{U} \subset \mathbb{R}^{n+1}$ be a bounded open set. Later, $\tilde{U}$ will be an open set which is very close to $U$ in Assumption 1.1.

Definition 3.2 For $N \geq 2$, an open partition of $\tilde{U}$ in $N$ elements is a finite and ordered collection $\mathcal{E}=\left\{E_{i}\right\}_{i=1}^{N}$ of subsets $E_{i} \subset \tilde{U}$ such that:

(a) $E_{1}, \ldots, E_{N}$ are open and mutually disjoint;

(b) $\mathcal{H}^{n}\left(\tilde{U} \backslash \bigcup_{i=1}^{N} E_{i}\right)<\infty$;

(c) $\bigcup_{i=1}^{N} \partial E_{i} \subset \tilde{U}$ is countably $n$-rectifiable.

The set of all open partitions of $\tilde{U}$ of $N$ elements will be denoted $\mathcal{O P}^{N}(\tilde{U})$.

Note that some of the $E_{i}$ may be empty. Condition (b) implies that

$$
\tilde{U} \backslash \bigcup_{i=1}^{N} E_{i}=\bigcup_{i=1}^{N} \partial E_{i},
$$

and thus that $\bigcup_{i=1}^{N} \partial E_{i}$ is $\mathcal{H}^{n}$-rectifiable and each $E_{i}$ is in fact an open set with finite perimeter in $\tilde{U}$. By De Giorgi's structure theorem, the reduced boundary $\partial^{*} E_{i}$ is $\mathcal{H}^{n}$-rectifiable: nonetheless, the reduced boundary $\partial^{*} E_{i}$ may not coincide in general with the topological boundary $\partial E_{i}$, which makes condition (c) not redundant. We keep the following for later use. The proof is straightforward.

Lemma 3.3 Suppose $\mathcal{E}=\left\{E_{i}\right\}_{i=1}^{N} \in \mathcal{O P}^{N}(\tilde{U})$ and $f: \mathbb{R}^{n+1} \rightarrow \mathbb{R}^{n+1}$ is a $C^{1}$ diffeomorphism. Then we have $\left\{f\left(E_{i}\right)\right\}_{i=1}^{N} \in \mathcal{O P}^{N}(f(\tilde{U}))$. 
Notation Given $\mathcal{E} \in \mathcal{O} \mathcal{P}^{N}(\tilde{U})$, we will set

$$
\partial \mathcal{E}:=\operatorname{var}\left(\bigcup_{i=1}^{N} \partial E_{i}, 1\right) \in \mathbf{I V}_{n}\left(\mathbb{R}^{n+1}\right) .
$$

Here, to avoid some possible confusion, we emphasize that we want to consider $\partial \mathcal{E}$ as a varifold on $\mathbb{R}^{n+1}$ when we construct approximate MCF. On the other hand, note that we still consider the relative topology of $\tilde{U}$, as $\partial E_{i} \subset \tilde{U}$ here. In particular, writing $\Gamma=\cup_{i=1}^{N} \partial E_{i}$, we have $\|\partial \mathcal{E}\|=\mathcal{H}^{n}\left\llcorner_{\Gamma}\right.$, and

$$
\partial \mathcal{E}(\varphi)=\int_{\Gamma} \varphi\left(x, T_{x} \Gamma\right) d \mathcal{H}^{n}(x) \quad \text { for every } \varphi \in C_{c}\left(\mathbf{G}_{n}\left(\mathbb{R}^{n+1}\right)\right),
$$

where $T_{x} \Gamma \in \mathbf{G}(n+1, n)$ is the approximate tangent plane to $\Gamma$ at $x$, which exists and is unique at $\mathcal{H}^{n}$-a.e. $x \in \Gamma$ because of Definition 3.2(c).

Definition 3.4 Given $\mathcal{E}=\left\{E_{i}\right\}_{i=1}^{N} \in \mathcal{O} \mathcal{P}^{N}(\tilde{U})$ and a closed set $C \subset \subset \tilde{U}$, a function $f: \mathbb{R}^{n+1} \rightarrow \mathbb{R}^{n+1}$ is $\mathcal{E}$-admissible in $C$ if it is Lipschitz continuous and satisfies the following. Let $\tilde{E}_{i}:=\operatorname{int}\left(f\left(E_{i}\right)\right)$ for $i=1, \ldots, N$. Then:

(a) $\{x: x \neq f(x)\} \cup\{f(x): x \neq f(x)\} \subset C$;

(b) $\left\{\tilde{E}_{i}\right\}_{i=1}^{N}$ are mutually disjoint;

(c) $\tilde{U} \backslash \bigcup_{i=1}^{N} \tilde{E}_{i} \subset f\left(\bigcup_{i=1}^{N} \partial E_{i}\right)$.

Lemma 3.5 Let $\mathcal{E}=\left\{E_{i}\right\}_{i=1}^{N} \in \mathcal{O} \mathcal{P}^{N}(\tilde{U})$ be an open partition of $\tilde{U}$ in $N$ elements, $C \subset \subset \tilde{U}$, and let $f$ be $\mathcal{E}$-admissible in $C$. If we define $\tilde{\mathcal{E}}:=\left\{\tilde{E}_{i}\right\}_{i=1}^{N}$ with $\tilde{E}_{i}:=\operatorname{int}\left(f\left(E_{i}\right)\right)$, then $\tilde{\mathcal{E}} \in \mathcal{O P}^{N}(\tilde{U})$.

Proof We check that $\tilde{\mathcal{E}}$ satisfies properties (a)-(c) in Definition 3.2. By Definition 3.4(a) and (b), it is clear that $\tilde{E}_{1}, \ldots, \tilde{E}_{N}$ are open and mutually disjoint subsets of $\tilde{U}$, which gives (a). In order to prove (b), we use Definition 3.4(c) and the area formula to compute:

$$
\mathcal{H}^{n}\left(\tilde{U} \backslash \bigcup_{i=1}^{N} \tilde{E}_{i}\right) \leq \mathcal{H}^{n}\left(f\left(\bigcup_{i=1}^{N} \partial E_{i}\right)\right) \leq \operatorname{Lip}(f)^{n} \mathcal{H}^{n}\left(\bigcup_{i=1}^{N} \partial E_{i}\right)<\infty,
$$

where we have used Definition 3.2(b) and (3.7). This also shows $\tilde{U} \backslash \bigcup_{i=1}^{N} \tilde{E}_{i}=\bigcup_{i=1}^{N} \partial \tilde{E}_{i}$. Finally, we prove property (c). Observe that, since $\bigcup_{i=1}^{N} \partial E_{i}$ is countably $n$-rectifiable, also the set $f\left(\bigcup_{i=1}^{N} \partial E_{i}\right)$ is countably $n$-rectifiable. Since any subset of a countably $n$-rectifiable set is countably $n$-rectifiable, also $\bigcup_{i=1}^{N} \partial \tilde{E}_{i}$ is countably $n$-rectifiable.

Notation If $\mathcal{E} \in \mathcal{O} \mathcal{P}^{N}(\tilde{U})$ and $f \in \operatorname{Lip}\left(\mathbb{R}^{n+1} ; \mathbb{R}^{n+1}\right)$ is $\mathcal{E}$-admissible in $C$ for some $C \subset \subset \tilde{U}$, then the open partition $\tilde{\mathcal{E}} \in \mathcal{O} \mathcal{P}^{N}(\tilde{U})$ will be denoted $f_{\star} \mathcal{E}$.

\subsection{Area reducing Lipschitz deformations}

Definition 3.6 For $\mathcal{E}=\left\{E_{i}\right\}_{i=1}^{N} \in \mathcal{O} \mathcal{P}^{N}(\tilde{U}), j \in \mathbb{N}$ and a closed set $C \subset \subset \tilde{U}$, define $\mathbf{E}(\mathcal{E}, C, j)$ to be the set of all $\mathcal{E}$-admissible functions $f$ in $C$ such that:

(a) $|f(x)-x| \leq 1 / j^{2}$ for every $x \in C$;

(b) $\mathcal{L}^{n+1}\left(\tilde{E}_{i} \triangle E_{i}\right) \leq 1 / j$ for all $i=1, \ldots, N$, where $\tilde{E}_{i}=\operatorname{int}\left(f\left(E_{i}\right)\right)$, and where $E \triangle F:=$ $[E \backslash F] \cup[F \backslash E]$ is the symmetric difference of the sets $E$ and $F$; 
(c) $\left\|\partial f_{\star} \mathcal{E}\right\|(\phi) \leq\|\partial \mathcal{E}\|(\phi)$ for all $\phi \in \mathcal{A}_{j}$. Here, $f_{\star} \mathcal{E}=\left\{\tilde{E}_{i}\right\}_{i=1}^{N}$ and $\|\partial \mathcal{E}\|$ is the weight of the multiplicity one varifold associated to the open partition $\mathcal{E}$.

The set $\mathbf{E}(\mathcal{E}, C, j)$ is not empty, as it contains the identity map.

Definition 3.7 Given $\mathcal{E} \in \mathcal{O P}^{N}(\tilde{U})$ and $j$, and given a closed set $C \subset \subset \tilde{U}$, we define

$$
\begin{aligned}
\Delta_{j}\|\partial \mathcal{E}\|(C): & =\inf _{f \in \mathbf{E}(\mathcal{E}, C, j)}\left\{\left\|\partial f_{\star} \mathcal{E}\right\|(C)-\|\partial \mathcal{E}\|(C)\right\} \\
& =\inf _{f \in \mathbf{E}(\mathcal{E}, C, j)}\left\{\left\|\partial f_{\star} \mathcal{E}\right\|\left(\mathbb{R}^{n+1}\right)-\|\partial \mathcal{E}\|\left(\mathbb{R}^{n+1}\right)\right\} .
\end{aligned}
$$

Observe that it always holds $\Delta_{j}\|\partial \mathcal{E}\|(C) \leq 0$, since the identity map $f(x)=x$ belongs to $\mathbf{E}(\mathcal{E}, C, j)$. The quantity $\Delta_{j}\|\partial \mathcal{E}\|(C)$ measures the extent to which $\|\partial \mathcal{E}\|$ can be reduced by acting with area reducing Lipschitz deformations in $C$.

\subsection{Smoothing of varifolds and first variations}

We let $\psi \in C^{\infty}\left(\mathbb{R}^{n+1}\right)$ be a radially symmetric function such that

$$
\begin{aligned}
& \psi(x)=1 \text { for }|x| \leq 1 / 2, \quad \psi(x)=0 \text { for }|x| \geq 1, \\
& 0 \leq \psi(x) \leq 1, \quad|\nabla \psi(x)| \leq 3, \quad\left\|\nabla^{2} \psi(x)\right\| \leq 9 \text { for all } x \in \mathbb{R}^{n+1},
\end{aligned}
$$

and we define, for each $\varepsilon \in(0,1)$,

$$
\hat{\Phi}_{\varepsilon}(x):=\frac{1}{\left(2 \pi \varepsilon^{2}\right)^{\frac{n+1}{2}}} \exp \left(-\frac{|x|^{2}}{2 \varepsilon^{2}}\right), \quad \Phi_{\varepsilon}(x):=c(\varepsilon) \psi(x) \hat{\Phi}_{\varepsilon}(x),
$$

where the constant $c(\varepsilon)$ is chosen in such a way that

$$
\int_{\mathbb{R}^{n+1}} \Phi_{\varepsilon}(x) d x=1 .
$$

The function $\Phi_{\varepsilon}$ will be adopted as a convolution kernel for the definition of the smoothing of a varifold. We record the properties of $\Phi_{\varepsilon}$ in the following lemma (cf. [20, Lemma 4.13]).

Lemma 3.8 There exists a constant $c=c(n)$ such that, for $\varepsilon \in(0,1)$, we have:

$$
\begin{aligned}
\left|\nabla \Phi_{\varepsilon}(x)\right| & \leq \frac{|x|}{\varepsilon^{2}} \Phi_{\varepsilon}(x)+c \chi_{B_{1} \backslash B_{1 / 2}}(x) \exp \left(-\varepsilon^{-1}\right), \\
\left\|\nabla^{2} \Phi_{\varepsilon}(x)\right\| & \leq \frac{|x|^{2}}{\varepsilon^{4}} \Phi_{\varepsilon}(x)+\frac{c}{\varepsilon^{2}} \Phi_{\varepsilon}(x)+c \chi_{B_{1} \backslash B_{1 / 2}}(x) \exp \left(-\varepsilon^{-1}\right) .
\end{aligned}
$$

Next, we use the convolution kernel $\Phi_{\varepsilon}$ in order to define the smoothing of a varifold and its first variation. Recall that, given a Radon measure $\mu$ on $\mathbb{R}^{n+1}$, the smoothing of $\mu$ by means of the kernel $\Phi_{\varepsilon}$ is defined to be the Radon measure $\Phi_{\varepsilon} * \mu$ given by

$$
\begin{aligned}
& \left(\Phi_{\varepsilon} * \mu\right)(\phi):=\mu\left(\Phi_{\varepsilon} * \phi\right) \\
& =\int_{\mathbb{R}^{n+1}} \int_{\mathbb{R}^{n+1}} \Phi_{\varepsilon}(x-y) \phi(y) d y d \mu(x) \quad \text { for every } \phi \in C_{c}\left(\mathbb{R}^{n+1}\right) .
\end{aligned}
$$

The definition of smoothing of a varifold $V$ is the equivalent of (3.15) when regarding $V$ as a Radon measure on $\mathbf{G}_{n}\left(\mathbb{R}^{n+1}\right)$, keeping in mind that the operator $\left(\Phi_{\varepsilon} *\right)$ acts on a test function $\varphi \in C_{c}\left(\mathbf{G}_{n}\left(\mathbb{R}^{n+1}\right)\right)$ by convolving only the space variable. Explicitly, we give the following definition. 
Definition 3.9 Given $V \in \mathbf{V}_{n}\left(\mathbb{R}^{n+1}\right)$, we let $\Phi_{\varepsilon} * V \in \mathbf{V}_{n}\left(\mathbb{R}^{n+1}\right)$ be the varifold defined by

$$
\left(\Phi_{\varepsilon} * V\right)(\varphi):=V\left(\Phi_{\varepsilon} * \varphi\right)=\int_{\mathbf{G}_{n}\left(\mathbb{R}^{n+1}\right)} \int_{\mathbb{R}^{n+1}} \Phi_{\varepsilon}(x-y) \varphi(y, S) d y d V(x, S)
$$

for every $\varphi \in C_{c}\left(\mathbf{G}_{n}\left(\mathbb{R}^{n+1}\right)\right)$.

Observe that, given a Radon measure $\mu$ on $\mathbb{R}^{n+1}$, one can identify the measure $\Phi_{\varepsilon} * \mu$ with a $C^{\infty}$ function by means of the Hilbert space structure of $L^{2}\left(\mathbb{R}^{n+1}\right)=L^{2}\left(\mathcal{L}^{n+1}\right)$. Indeed, for any $\phi \in C_{c}\left(\mathbb{R}^{n+1}\right)$ we have that

$$
\left(\Phi_{\varepsilon} * \mu\right)(\phi)=\left\langle\Phi_{\varepsilon} * \mu \mid \phi\right\rangle_{L^{2}\left(\mathbb{R}^{n+1}\right)},
$$

where $\Phi_{\varepsilon} * \mu \in C^{\infty}\left(\mathbb{R}^{n+1}\right)$ is defined by

$$
\left(\Phi_{\varepsilon} * \mu\right)(x):=\int_{\mathbb{R}^{n+1}} \Phi_{\varepsilon}(x-y) d \mu(y) .
$$

These considerations suggest the following definition for the smoothing of the first variation of a varifold.

Definition 3.10 Given $V \in \mathbf{V}_{n}\left(\mathbb{R}^{n+1}\right)$, the smoothing of $\delta V$ by means of the convolution kernel $\Phi_{\varepsilon}$ is the vector field $\Phi_{\varepsilon} * \delta V \in C^{\infty}\left(\mathbb{R}^{n+1} ; \mathbb{R}^{n+1}\right)$ defined by

$$
\left(\Phi_{\varepsilon} * \delta V\right)(x):=\int_{\mathbf{G}_{n}\left(\mathbb{R}^{n+1}\right)} S\left(\nabla \Phi_{\varepsilon}(y-x)\right) d V(y, S),
$$

in such a way that

$$
\delta V\left(\Phi_{\varepsilon} * g\right)=\left\langle\Phi_{\varepsilon} * \delta V \mid g\right\rangle_{L^{2}\left(\mathbb{R}^{n+1}\right)} \quad \text { for every } g \in C_{c}^{1}\left(\mathbb{R}^{n+1} ; \mathbb{R}^{n+1}\right) .
$$

Lemma 3.11 For $V \in \mathbf{V}_{n}\left(\mathbb{R}^{n+1}\right)$, we have

$$
\begin{aligned}
\Phi_{\varepsilon} *\|V\| & =\left\|\Phi_{\varepsilon} * V\right\|, \\
\Phi_{\varepsilon} * \delta V & =\delta\left(\Phi_{\varepsilon} * V\right) .
\end{aligned}
$$

Moreover, if $\|V\|\left(\mathbb{R}^{n+1}\right)<\infty$ then

$$
\left\|\Phi_{\varepsilon} * V\right\|\left(\mathbb{R}^{n+1}\right) \leq\|V\|\left(\mathbb{R}^{n+1}\right) .
$$

Proof The identities (3.19) and (3.20) are proved in [20, Lemma 4.16]. Concerning (3.21), we observe that for any $\varphi \in C_{c}\left(\mathbf{G}_{n}\left(\mathbb{R}^{n+1}\right)\right)$ with $\|\varphi\|_{0} \leq 1$, setting $\tau_{z}(x):=x-z$, it holds:

$$
\begin{aligned}
\left(\Phi_{\varepsilon} * V\right)(\varphi) & =\int_{\mathbf{G}_{n}\left(\mathbb{R}^{n+1}\right)} \int_{\mathbb{R}^{n+1}} \Phi_{\varepsilon}(x-y) \varphi(y, S) d y d V(x, S) \\
& =\int_{\mathbf{G}_{n}\left(\mathbb{R}^{n+1}\right)} \int_{\mathbb{R}^{n+1}} \Phi_{\varepsilon}(z) \varphi(x-z, S) d z d V(x, S) \\
& =\int_{\mathbb{R}^{n+1}} \Phi_{\varepsilon}(z) \int_{\mathbf{G}_{n}\left(\mathbb{R}^{n+1}\right)} \varphi\left(\tau_{z}(x), S\right) d V(x, S) d z \leq\|V\|\left(\mathbb{R}^{n+1}\right) .
\end{aligned}
$$

Taking the supremum among all functions $\varphi \in C_{c}\left(\mathbf{G}_{n}\left(\mathbb{R}^{n+1}\right)\right)$ with $\|\varphi\|_{0} \leq 1$ completes the proof. 


\subsection{Smoothed mean curvature vector}

Definition 3.12 Given $V \in \mathbf{V}_{n}\left(\mathbb{R}^{n+1}\right)$ and $\varepsilon \in(0,1)$, the smoothed mean curvature vector of $V$ is the vector field $h_{\varepsilon}(\cdot, V) \in C^{\infty}\left(\mathbb{R}^{n+1} ; \mathbb{R}^{n+1}\right)$ defined by

$$
h_{\varepsilon}(\cdot, V):=-\Phi_{\varepsilon} *\left(\frac{\Phi_{\varepsilon} * \delta V}{\Phi_{\varepsilon} *\|V\|+\varepsilon}\right) .
$$

We will often make use of [20, Lemma 5.1] with $\Omega \equiv 1$ (and $c_{1}=0$ ). For the reader's convenience, we provide here the statement.

Lemma 3.13 For every $M>0$, there exists a constant $\varepsilon_{1} \in(0,1)$, depending only on $n$ and $M$ such that the following holds. Let $V \in \mathbf{V}_{n}\left(\mathbb{R}^{n+1}\right)$ be an $n$-dimensional varifold in $\mathbb{R}^{n+1}$ such that $\|V\|\left(\mathbb{R}^{n+1}\right) \leq M$, and, for every $\varepsilon \in\left(0, \varepsilon_{1}\right)$, let $h_{\varepsilon}(\cdot, V)$ be its smoothed mean curvature vector. Then:

$$
\begin{aligned}
& \left|h_{\varepsilon}(x, V)\right| \leq 2 \varepsilon^{-2}, \\
& \left\|\nabla h_{\varepsilon}(x, V)\right\| \leq 2 \varepsilon^{-4}, \\
& \left\|\nabla^{2} h_{\varepsilon}(x, V)\right\| \leq 2 \varepsilon^{-6} .
\end{aligned}
$$

\subsection{The cut-off functions $\eta_{j}$}

In this subsection we construct the cut-off functions which will later be used to truncate the smoothed mean curvature vector in order to produce time-discrete approximate flows which almost preserve the boundary $\partial \Gamma_{0}$.

Given a set $E \subset \mathbb{R}^{n+1}$ and $s>0,(E)_{s}$ denotes the $s$-neighborhood of $E$, namely the open set

$$
(E)_{s}:=\bigcup_{x \in E} U_{s}(x)
$$

We shall also adopt the convention that $(E)_{0}=E$.

Let $U$ and $\Gamma_{0}$ be as in Assumption 1.1.

Definition 3.14 We define for $j \in \mathbb{N}$ :

$$
D_{j}:=\left\{x \in U: \operatorname{dist}(x, \partial U) \geq \frac{2}{j^{1 / 4}}\right\} .
$$

Observe that $D_{j}$ is not empty for all $j$ sufficiently large (depending on $U$ ).

Also, we define the sets

$$
K_{j}:=\left(\Gamma_{0} \backslash D_{j}\right)_{1 / j^{1 / 4}}, \quad \tilde{K}_{j}:=\left(\Gamma_{0} \backslash D_{j}\right)_{2 / j^{1 / 4}}, \quad \text { and } \quad \hat{K}_{j}:=\left(\Gamma_{0} \backslash D_{j}\right)_{3 / j^{1 / 8}}
$$

so that $K_{j} \subset \tilde{K}_{j} \subset \hat{K}_{j}$.

Definition 3.15 Let $\psi:(0, \infty) \rightarrow \mathbb{R}$ be a smooth function satisfying the following properties:

(a) $0 \leq \psi(t) \leq 1$ for every $t>0, \psi(t)=t$ for $t \in(0,1 / 2], t / 2 \leq \psi(t) \leq t$ for $t \in[1 / 2,3 / 2], \psi(t)=1$ for $t \geq 3 / 2$;

(b) $0 \leq \psi^{\prime}(t) \leq 1$ for every $t>0$;

(c) $\left|\psi^{\prime \prime}(t)\right| \leq 2$ for every $t>0$. 
For every $j \in \mathbb{N}$, set

$$
\hat{\mathrm{d}}_{j}(x):=\operatorname{dist}\left(x, \mathbb{R}^{n+1} \backslash\left(\Gamma_{0} \backslash D_{j}\right)_{2 / j^{1 / 8}}\right) \quad \text { for every } x \in \mathbb{R}^{n+1} .
$$

Let $\left\{\phi_{\rho}\right\}_{\rho}, \rho>0$, be a standard family of mollifiers: precisely, let

$$
\phi(w):= \begin{cases}A_{n} \exp \left(\frac{1}{|w|^{2}-1}\right) & \text { if }|w|<1 \\ 0 & \text { otherwise }\end{cases}
$$

for a suitable normalization constant $A_{n}$ chosen in such a way that $\int_{\mathbb{R}^{n+1}} \phi(w) d w=1$, and define $\phi_{\rho}(z):=\rho^{-(n+1)} \phi(z / \rho)$. Then, set $\rho_{j}:=1 /\left(j^{1 / 4}\right)$, and $\mathrm{d}_{j}:=\phi_{\rho_{j}} * \hat{\mathrm{d}}_{j}$. We finally define

$$
\eta_{j}(x):=\psi\left(\exp \left(-j^{1 / 4}\left(\mathrm{~d}_{j}(x)-j^{-1 / 4}\right)\right)\right) .
$$

Lemma 3.16 There exists $J=J(n)$ such that the following properties hold for all $j \geq J$ :

(1) $\eta_{j} \equiv 1$ on $\mathbb{R}^{n+1} \backslash \hat{K}_{j}$;

(2) $0<\eta_{j} \leq \exp \left(-j^{1 / 8}\right)$ on $\tilde{K}_{j}$;

(3) $\eta_{j} \in \mathcal{A}_{j^{3 / 4}}$

Proof For the proof of (1), if $x \notin \hat{K}_{j}$ then $\hat{\mathrm{d}}_{j}(x)=0$. Moreover, since $\rho_{j}=j^{-1 / 4}<j^{-1 / 8}$, evidently $\hat{\mathrm{d}}_{j}(y)=0$ for all $y \in B_{\rho_{j}}(x)$. This implies that

$$
\mathrm{d}_{j}(x)=\left(\phi_{\rho_{j}} * \hat{\mathrm{d}}_{j}\right)(x)=\int_{B_{\rho_{j}}(x)} \phi_{\rho_{j}}(x-y) \hat{\mathrm{d}}_{j}(y) d y=0 .
$$

Hence, $\eta_{j}(x)=\psi(e)=1$ because of property (a) of $\psi$ in Definition 3.15.

Next, we prove (2). Let $x \in \tilde{K}_{j}$, so that there exists $z \in \Gamma_{0} \backslash D_{j}$ such that $|x-z|<2 j^{-1 / 4}$. If $y \in B_{\rho_{j}}(x)$, then $|y-z|<3 j^{-1 / 4}$ by the definition of $\rho_{j}$, and thus, for $j$ suitably large,

$$
\hat{\mathrm{d}}_{j}(y)=\operatorname{dist}\left(y, \mathbb{R}^{n+1} \backslash\left(\Gamma_{0} \backslash D_{j}\right)_{2 / j^{1 / 8}}\right) \geq 2 j^{-1 / 8}-3 j^{-1 / 4},
$$

which in turn implies

$$
\mathrm{d}_{j}(x)=\left(\phi_{\rho_{j}} * \hat{\mathrm{d}}_{j}\right)(x)=\int_{B_{\rho_{j}}(x)} \phi_{\rho_{j}}(x-y) \hat{\mathrm{d}}_{j}(y) \geq 2 j^{-1 / 8}-3 j^{-1 / 4} .
$$

Hence, setting $t:=\exp \left(-j^{1 / 4}\left(\mathrm{~d}_{j}(x)-j^{-1 / 4}\right)\right)$ we have that $0<t \leq \exp \left(4-2 j^{1 / 8}\right) \leq 1 / 2$ for $j$ large enough. Hence, by property (a) of $\psi$ in Definition 3.15:

$$
\eta_{j}(x)=\psi(t)=t \leq \exp \left(4-2 j^{1 / 8}\right) \quad \text { for every } x \in \tilde{K}_{j} .
$$

In particular, up to taking larger values of $j$, we see that

$$
0<\eta_{j}(x) \leq e^{-j^{1 / 8}} \quad \text { for every } x \in \tilde{K}_{j} .
$$

Finally, we prove (3). To this aim, we compute the gradient of $\eta_{j}$ : at any point $x$, we have

$$
\nabla \eta_{j}=-j^{1 / 4} \psi^{\prime}(t) t \nabla \mathrm{d}_{j} .
$$

Using that $t=\psi(t)$ for $0 \leq t \leq 1 / 2, \psi^{\prime}(t)=0$ for $t \geq 3 / 2$, and that $|t|=t \leq 2 \psi(t)$ for $t \in[1 / 2,3 / 2]$, together with the fact that $\left|\psi^{\prime}\right| \leq 1$, we can estimate

$$
\left|\nabla \eta_{j}\right| \leq 2 j^{1 / 4}\left|\nabla \mathrm{d}_{j}\right| \eta_{j} \leq 2 j^{1 / 4} \eta_{j},
$$


where we have used that $\nabla \mathrm{d}_{j}(x)=\phi_{\rho_{j}} * \nabla \hat{d}_{j}(x)$, so that

$$
\left|\nabla \mathrm{d}_{j}(x)\right| \leq \int_{B_{\rho_{j}}(x)} \phi_{\rho_{j}}(x-y)\left|\nabla \hat{\mathrm{d}}_{j}(y)\right| \leq 1 .
$$

In particular, $\left|\nabla \eta_{j}\right| \leq j^{3 / 4} \eta_{j}$ as soon as $j \geq 4$. Next, we compute the Hessian of $\eta_{j}$

$$
\nabla^{2} \eta_{j}=j^{1 / 2} t\left(t \psi^{\prime \prime}(t)+\psi^{\prime}(t)\right) \nabla \mathrm{d}_{j} \otimes \nabla \mathrm{d}_{j}-j^{1 / 4} \psi^{\prime}(t) t \nabla^{2} \mathrm{~d}_{j},
$$

from which we estimate

$$
\left\|\nabla^{2} \eta_{j}\right\| \leq 100 j^{1 / 2} \eta_{j}+j^{1 / 4} \eta_{j}\left\|\nabla^{2} \mathrm{~d}_{j}\right\| .
$$

Now, observe that

$$
\begin{aligned}
\left\|\nabla^{2} \mathrm{~d}_{j}\right\| & \leq \int_{B_{\rho_{j}}(x)}\left\|\nabla \phi_{\rho_{j}}(x-y) \otimes \nabla \hat{\mathrm{d}}_{j}(y)\right\| d y \leq \int_{B_{\rho_{j}}}\left|\nabla \phi_{\rho_{j}}(z)\right| d z \\
& =\rho_{j}^{-1} \int_{B_{1}}|\nabla \phi(w)| d w=C(n) \rho_{j}^{-1} .
\end{aligned}
$$

Hence, recalling that $\rho_{j}=j^{-1 / 4}$, we conclude the estimate

$$
\left\|\nabla^{2} \eta_{j}\right\| \leq C(n) j^{1 / 2} \eta_{j}
$$

for a constant $C$ depending only on $n$. Thus, we conclude $\eta_{j} \in \mathcal{A}_{j^{3 / 4}}$ for $j$ sufficiently large.

\section{7 $L^{2}$ approximations}

In this subsection, we collect a few estimates of the error terms deriving from working with smoothed first variations and smoothed mean curvature vectors. They will be critically important to deduce the convergence of the discrete approximation algorithm. The first estimate is a modification of [20, Proposition 5.3]. We let $\eta_{j}$ be the cut-off function as in Definition 3.15 , corresponding to $U$ and $\Gamma_{0}$, and we will suppose that $j \geq J(n)$, in such a way that the conclusions of Lemma 3.16 are satisfied.

Proposition 3.17 For every $M>0$, there exists $\varepsilon_{2} \in(0,1)$ depending only on $n$ and $M$ such that the following holds. For any $j \geq J(n), g \in \mathcal{B}_{j}, V \in \mathbf{V}_{n}\left(\mathbb{R}^{n+1}\right)$ with $\|V\|\left(\mathbb{R}^{n+1}\right) \leq M$, $\varepsilon \in\left(0, \varepsilon_{2}\right)$ with

$$
j \leq \frac{1}{2} \varepsilon^{-\frac{1}{6}}
$$

we have for $h_{\varepsilon}(\cdot)=h_{\varepsilon}(\cdot, V)$ :

$$
\begin{aligned}
& \left|\int_{\mathbb{R}^{n+1}} h_{\varepsilon} \cdot \eta_{j} g d\|V\|+\int_{\mathbb{R}^{n+1}}\left(\Phi_{\varepsilon} * \delta V\right) \cdot \eta_{j} g d x\right| \\
& \leq \varepsilon^{\frac{1}{4}}\left(\int_{\mathbb{R}^{n+1}} \eta_{j} \frac{\left|\Phi_{\varepsilon} * \delta V\right|^{2}}{\Phi_{\varepsilon} *\|V\|+\varepsilon} d x\right)^{\frac{1}{2}} .
\end{aligned}
$$

Given the validity of (3.18), we see that (3.32) measures the deviation from the identity (2.5). The difference with [20, Proposition 5.3] is that there, in place of $\eta_{j} g$ (left-hand side of (3.32)) and $\eta_{j}$ (right-hand side of (3.32)), we have $g$ and $\Omega$, respectively. We note that $g \eta_{j}$ 
satisfies $\left|\left(g \eta_{j}\right)(x)\right| \leq j \eta_{j}(x)$ and $\left\|\nabla\left(g \eta_{j}\right)(x)\right\| \leq 2 j^{7 / 4} \eta_{j}(x)$ : using these, the modification of the proof is straightforward, and thus we omit the details.

The following is [20, Proposition 5.4].

Proposition 3.18 There exists a constant $\varepsilon_{3} \in(0,1)$ depending only on $n$ and $M$ with the following property. Given any $V \in \mathbf{V}_{n}\left(\mathbb{R}^{n+1}\right)$ with $\|V\|\left(\mathbb{R}^{n+1}\right) \leq M, j \in \mathbb{N}, \phi \in \mathcal{A}_{j}$, and $\varepsilon \in\left(0, \varepsilon_{3}\right)$ satisfying (3.31), we have:

$$
\begin{aligned}
\mid \delta V\left(\phi h_{\varepsilon}\right)+\int_{\mathbb{R}^{n+1}} \phi & \frac{\left|\Phi_{\varepsilon} * \delta V\right|^{2}}{\Phi_{\varepsilon} *\|V\|+\varepsilon} d x \mid \leq \varepsilon^{\frac{1}{4}}\left(\int_{\mathbb{R}^{n+1}} \phi \frac{\left|\Phi_{\varepsilon} * \delta V\right|^{2}}{\Phi_{\varepsilon} *\|V\|+\varepsilon} d x+1\right), \\
\int_{\mathbb{R}^{n+1}}\left|h_{\varepsilon}\right|^{2} \phi d\|V\| & \leq\left(1+\varepsilon^{\frac{1}{4}}\right) \int_{\mathbb{R}^{n+1}} \phi \frac{\left|\Phi_{\varepsilon} * \delta V\right|^{2}}{\Phi_{\varepsilon} *\|V\|+\varepsilon} d x+\varepsilon^{\frac{1}{4}} .
\end{aligned}
$$

Note that formula (3.33) estimates the deviation from the identity (2.5) with $g=h(\cdot, V)$.

The next statement is [20, Proposition 5.5]. The proof is a straightforward modification, using (3.32).

Proposition 3.19 For every $M>0$, there exists $\varepsilon_{4} \in(0,1)$ depending only on $n$ and $M$ with the following property. For any $j \geq J(n), g \in \mathcal{B}_{j}, V \in \mathbf{V}_{n}\left(\mathbb{R}^{n+1}\right)$ with $\|V\|\left(\mathbb{R}^{n+1}\right) \leq M$, $\varepsilon \in\left(0, \varepsilon_{4}\right)$ satisfying (3.31), it holds

$$
\left|\int_{\mathbb{R}^{n+1}} h_{\varepsilon} \cdot \eta_{j} g d\|V\|+\delta V\left(\eta_{j} g\right)\right| \leq \varepsilon^{\frac{1}{4}}\left(1+\left(\int_{\mathbb{R}^{n+1}} \eta_{j} \frac{\left|\Phi_{\varepsilon} * \delta V\right|^{2}}{\Phi_{\varepsilon} *\|V\|+\varepsilon} d x\right)^{\frac{1}{2}}\right) .
$$

\subsection{Curvature of limit varifolds}

The next Proposition 3.20 corresponds to [20, Proposition 5.6] when there is no boundary.

Proposition 3.20 Suppose that $\left\{V_{j_{\ell}}\right\}_{\ell=1}^{\infty} \subset \mathbf{V}_{n}\left(\mathbb{R}^{n+1}\right)$ and $\left\{\varepsilon_{j_{\ell}}\right\}_{\ell=1}^{\infty} \subset(0,1)$ are such that:

(1) $\sup _{\ell}\left\|V_{j_{\ell}}\right\|\left(\mathbb{R}^{n+1}\right)<\infty$,

(2) $\liminf \inf _{\ell \rightarrow \infty} \int_{\mathbb{R}^{n+1}} \eta_{j_{\ell}} \frac{\left|\Phi_{\varepsilon_{j_{\ell}}} * \delta V_{j_{\ell}}\right|^{2}}{\Phi_{\varepsilon_{j_{\ell}}} *\left\|V_{j_{\ell}}\right\|+\varepsilon_{j_{\ell}}} d x<\infty$,

(3) $\lim _{\ell \rightarrow \infty} \varepsilon_{j \ell}=0$ and $j_{\ell} \leq \varepsilon_{j_{\ell}}^{-\frac{1}{6}} / 2$.

Then, there exists a subsequence $\left\{j_{\ell}^{\prime}\right\} \subset\left\{j_{\ell}\right\}$ such that $V_{j_{\ell}^{\prime}} \rightarrow V \in \mathbf{V}_{n}\left(\mathbb{R}^{n+1}\right)$ in the sense of varifolds, and $V$ has a generalized mean curvature vector $h(\cdot, V)$ in $U$ such that

$$
\int_{U}|h(\cdot, V)|^{2} \phi d\|V\| \leq \liminf _{\ell \rightarrow \infty} \int_{\mathbb{R}^{n+1}} \eta_{j_{\ell}} \phi \frac{\left|\Phi_{\varepsilon_{j_{\ell}}} * \delta V_{j_{\ell}}\right|^{2}}{\Phi_{\varepsilon_{j_{\ell}}} *\left\|V_{j_{\ell}}\right\|+\varepsilon_{j_{\ell}}} d x
$$

for every $\phi \in C_{c}\left(U ; \mathbb{R}^{+}\right)$.

Proof By (1), we may choose a (not relabeled) subsequence $V_{j_{\ell}}$ converging to $V$ as varifolds on $\mathbb{R}^{n+1}$, and we may assume that the integrals in (2) for this subsequence converge to the lim inf of the original sequence. Fix $g \in C_{c}^{2}\left(U ; \mathbb{R}^{n+1}\right)$. For all sufficiently large $\ell$, we 
have $g \eta_{j_{\ell}}=g$ due to Lemma 3.16(1), (3.27) and (3.26). Moreover, we may assume that $g \eta_{j_{\ell}} \in \mathcal{B}_{j_{\ell}}$ due to Lemma 3.16(3). Then, by (3.35), (2) and (3), we have

$$
\delta V(g)=\lim _{\ell \rightarrow \infty} \delta V_{j_{\ell}}\left(g \eta_{j_{\ell}}\right)=-\lim _{\ell \rightarrow \infty} \int_{\mathbb{R}^{n+1}} h_{\varepsilon_{j_{\ell}}}\left(\cdot, V_{j_{\ell}}\right) \cdot \eta_{j_{\ell}} g d\left\|V_{j_{\ell}}\right\| .
$$

Since $\eta_{j_{\ell}} \in \mathcal{A}_{j_{\ell}}$ in particular, by the Cauchy-Schartz inequality and (3.34), we have

$$
\delta V(g) \leq\left(\liminf _{\ell \rightarrow \infty} \int_{\mathbb{R}^{n+1}} \frac{\left|\Phi_{\varepsilon_{j_{\ell}}} * \delta V_{j_{\ell}}\right|^{2} \eta_{j_{\ell}}}{\Phi_{\varepsilon_{j_{\ell}}} *\left\|V_{j_{\ell}}\right\|+\varepsilon_{j_{\ell}}} d x\right)^{1 / 2}\left(\int_{\mathbb{R}^{n+1}}|g|^{2} d\|V\|\right)^{1 / 2} .
$$

This shows that $\delta V$ is absolutely continuous with respect to $\|V\|$ on $U$ and $h(\cdot, V)$ satisfies

$$
\int_{U}|h(\cdot, V)|^{2} d\|V\| \leq \liminf _{\ell \rightarrow \infty} \int_{\mathbb{R}^{n+1}} \frac{\left|\Phi_{\varepsilon_{j_{\ell}}} * \delta V_{j_{\ell}}\right|^{2} \eta_{j_{\ell}}}{\Phi_{\varepsilon_{j_{\ell}}} *\left\|V_{j_{\ell}}\right\|+\varepsilon_{j_{\ell}}} d x
$$

Given $\phi \in C_{c}^{2}\left(U ; \mathbb{R}^{+}\right)\left(C_{c}\right.$ case is by approximation), let $i \in \mathbb{N}$ be arbitrary and consider $\hat{\phi}:=\phi+i^{-1}$. For all sufficiently large $\ell$, we have $g \eta_{j_{\ell}} \hat{\phi} \in \mathcal{B}_{j_{\ell}}$ and $\eta_{j_{\ell}} \hat{\phi} \in \mathcal{A}_{j_{\ell}}$ (we may assume $|\hat{\phi}|<1$ without loss of generality). Thus the same computation above with $g \eta_{j_{\ell}} \hat{\phi}$ yields

$$
\int_{\mathbb{R}^{n+1}} h \cdot g \hat{\phi} d\|V\| \leq\left(\liminf _{\ell \rightarrow \infty} \int_{\mathbb{R}^{n+1}} \frac{\left|\Phi_{\varepsilon_{j_{\ell}}} * \delta V_{j_{\ell}}\right|^{2} \eta_{j_{\ell}} \hat{\phi}}{\Phi_{\varepsilon_{j_{\ell}}} *\left\|V_{j_{\ell}}\right\|+\varepsilon_{j_{\ell}}} d x\right)^{1 / 2}\left(\int_{\mathbb{R}^{n+1}}|g|^{2} \hat{\phi} d\|V\|\right)^{1 / 2} .
$$

We let then $i \rightarrow \infty$ in (3.40) to replace $\hat{\phi}$ by $\phi$, and finally we approximate $h(\cdot, V)$ by $g$ to obtain (3.36).

\subsection{Motion by smoothed mean curvature with boundary damping}

We aim at proving the following proposition: it contains the perturbation estimates for a varifold $V$ which is moved by a vector field consisting of a boundary damping of its smoothed mean curvature for a time $\Delta t$.

Proposition 3.21 There exists $\varepsilon_{5} \in(0,1)$, depending only on $n, M$ and $U$ such that the following holds. Suppose that:

(1) $V \in \mathbf{V}_{n}\left(\mathbb{R}^{n+1}\right)$ satisfies $\mathrm{spt}\|V\| \subset(U)_{1}$ and $\|V\|\left(\mathbb{R}^{n+1}\right) \leq M$;

(2) $j \geq J(n)$ and $\eta_{j}$ is as in Definition 3.15;

(3) $\varepsilon \in\left(0, \varepsilon_{5}\right)$ satisfies (3.31);

(4) $\Delta t \in\left[2^{-1} \varepsilon^{\kappa}, \varepsilon^{\kappa}\right]$, with

$$
\kappa=3 n+20 .
$$

Define

$$
f(x):=x+\eta_{j}(x) h_{\varepsilon}(x, V) \Delta t .
$$

Then, for every $\phi \in \mathcal{A}_{j}$ we have the following estimates.

$$
\begin{aligned}
& \left|\frac{\left\|f_{\sharp} V\right\|(\phi)-\|V\|(\phi)}{\Delta t}-\delta(V, \phi)\left(\eta_{j} h_{\varepsilon}(\cdot, V)\right)\right| \leq \varepsilon^{\kappa-10}, \\
& \frac{\left\|f_{\sharp} V\right\|\left(\mathbb{R}^{n+1}\right)-\|V\|\left(\mathbb{R}^{n+1}\right)}{\Delta t}+\frac{1}{4} \int_{\mathbb{R}^{n+1}} \eta_{j} \frac{\left|\Phi_{\varepsilon} * \delta V\right|^{2}}{\Phi_{\varepsilon} *\|V\|+\varepsilon} d x \leq 2 \varepsilon^{1 / 4} .
\end{aligned}
$$


Furthermore, if also $\left\|f_{\sharp} V\right\|\left(\mathbb{R}^{n+1}\right) \leq M$, then we have

$$
\begin{aligned}
& \left|\delta(V, \phi)\left(\eta_{j} h_{\varepsilon}(\cdot, V)\right)-\delta\left(f_{\sharp} V, \phi\right)\left(\eta_{j} h_{\varepsilon}\left(\cdot, f_{\sharp} V\right)\right)\right| \leq \varepsilon^{\kappa-2 n-18}, \\
& \left|\int_{\mathbb{R}^{n+1}} \eta_{j} \frac{\left|\Phi_{\varepsilon} * \delta V\right|^{2}}{\Phi_{\varepsilon} *\|V\|+\varepsilon} d x-\int_{\mathbb{R}^{n+1}} \eta_{j} \frac{\left|\Phi_{\varepsilon} * \delta\left(f_{\sharp} V\right)\right|^{2}}{\Phi_{\varepsilon} *\left\|f_{\sharp} V\right\|+\varepsilon} d x\right| \leq \varepsilon^{\kappa-3 n-18} .
\end{aligned}
$$

Proof. We want to estimate the following quantity

$$
\begin{aligned}
A & :=\left\|f_{\sharp} V\right\|(\phi)-\|V\|(\phi)-\delta(V, \phi)\left(\eta_{j} h_{\varepsilon}(\cdot, V)\right) \Delta t \\
& =\left\|f_{\sharp} V\right\|(\phi)-\|V\|(\phi)-\delta(V, \phi)(F),
\end{aligned}
$$

where $F(x):=\eta_{j}(x) h_{\varepsilon}(x, V) \Delta t=f(x)-x$. By (2.2) and (2.8), we have that

$$
A=\int_{\mathbf{G}_{n}\left(\mathbb{R}^{n+1}\right)}\left\{\phi(f(x))\left|\Lambda_{n} \nabla f(x) \circ S\right|-\phi(x)-\phi(x) \nabla F \cdot S-F \cdot \nabla \phi\right\} d V(x, S),
$$

which can be written as

$$
A=I_{1}+I_{2}+I_{3}
$$

with

$$
\begin{aligned}
& I_{1}:=\int_{\mathbf{G}_{n}\left(\mathbb{R}^{n+1}\right)}(\phi(f(x))-\phi(x))\left(\left|\Lambda_{n} \nabla f(x) \circ S\right|-1\right) d V(x, S), \\
& I_{2}:=\int_{\mathbf{G}_{n}\left(\mathbb{R}^{n+1}\right)} \phi(x)\left(\left|\Lambda_{n} \nabla f(x) \circ S\right|-1-\nabla F \cdot S\right) d V(x, S), \\
& I_{3}:=\int_{\mathbf{G}_{n}\left(\mathbb{R}^{n+1}\right)} \phi(f(x))-\phi(x)-\nabla \phi(x) \cdot F(x) d V(x, S) .
\end{aligned}
$$

Choose $\varepsilon_{5} \leq \min \left\{\varepsilon_{1}, \varepsilon_{3}\right\}$, so that the conclusions of Lemma 3.13 and Proposition 3.18 hold with $\varepsilon \in\left(0, \varepsilon_{5}\right)$. In order to estimate the size of the various integrands appearing in the definition of $I_{1}, I_{2}$ and $I_{3}$, we first observe that, by (3.23) and our assumption on $\Delta t$,

$$
|F(x)|=\left|\eta_{j} h_{\varepsilon}(\cdot, V) \Delta t\right| \leq 2 \varepsilon^{\kappa-2} .
$$

Furthermore, using (3.23), (3.24), (3.31), and the fact that $\eta_{j} \in \mathcal{A}_{j}$ we obtain

$$
\|\nabla F\| \leq \Delta t\left(\eta_{j}\left\|\nabla h_{\varepsilon}\right\|+\left\|h_{\varepsilon} \otimes \nabla \eta_{j}\right\|\right) \leq \varepsilon^{\kappa}\left(2 \varepsilon^{-4}+2 j \varepsilon^{-2}\right) \leq 3 \varepsilon^{\kappa-4} .
$$

Since $\phi \in \mathcal{A}_{j}$, we can use the results of Lemma 3.1 to estimate:

$$
\begin{gathered}
|\phi(f(x))-\phi(x)| \stackrel{(3.4)}{\leq} j|F(x)| \phi(x) \exp (j|F(x)|) \leq \varepsilon^{\kappa-3}, \\
|\phi(f(x))-\phi(x)-\nabla \phi(x) \cdot F(x)| \stackrel{(3.5)}{\leq} j|F(x)|^{2} \phi(x) \exp (j|F(x)|) \leq \varepsilon^{\kappa-5} \Delta t .
\end{gathered}
$$

Analogously, using that $f(x)=x+F(x)$, so that

$$
\left|\Lambda_{n} \nabla f(x) \circ S\right|=\left|(\operatorname{Id}+\nabla F(x)) \cdot v_{1} \wedge \ldots \wedge(\mathrm{Id}+\nabla F(x)) \cdot v_{n}\right|
$$

for any orthonormal basis $\left\{v_{1}, \ldots, v_{n}\right\}$ of $S$, we can Taylor expand the tangential Jacobian and deduce the estimates

$$
|| \Lambda_{n} \nabla f(x) \circ S|-1| \leq c(n)\|\nabla F\| \stackrel{(3.46)}{\leq} c(n) \varepsilon^{\kappa-4} \leq c(n) \Delta t \varepsilon^{-4} \leq \Delta t \varepsilon^{-5},
$$




$$
|| \Lambda_{n} \nabla f(x) \circ S|-1-\nabla F \cdot S| \leq c(n)\|\nabla F\|^{2} \stackrel{(3.46)}{\leq} c(n) \varepsilon^{2 \kappa-8} \leq \varepsilon^{k-9} \Delta t,
$$

modulo choosing a smaller value of $\varepsilon$ if necessary. Putting all together, we can finally conclude the proof of (3.41):

$$
|A| \leq\left|I_{1}\right|+\left|I_{2}\right|+\left|I_{3}\right| \leq\left(\varepsilon^{\kappa-8}+\varepsilon^{\kappa-9}+\varepsilon^{\kappa-5}\right) \Delta t\|V\|\left(\mathbb{R}^{n+1}\right) \leq \varepsilon^{\kappa-10} \Delta t .
$$

In order to prove (3.42), we use (3.41) with $\phi(x) \equiv 1$, which implies that

$$
\frac{\left\|f_{\sharp} V\right\|\left(\mathbb{R}^{n+1}\right)-\|V\|\left(\mathbb{R}^{n+1}\right)}{\Delta t} \leq \delta V\left(\eta_{j} h_{\varepsilon}(\cdot, V)\right)+\varepsilon^{\kappa-10} .
$$

On the other hand, since $\eta_{j} \in \mathcal{A}_{j}$ we can apply (3.33) to further estimate

$$
\delta V\left(\eta_{j} h_{\varepsilon}\right) \leq-\left(1-\varepsilon^{1 / 4}\right)\left(\int_{\mathbb{R}^{n+1}} \eta_{j} \frac{\left|\Phi_{\varepsilon} * \delta V\right|^{2}}{\Phi_{\varepsilon} *\|V\|+\varepsilon} d x\right)+\varepsilon^{1 / 4},
$$

so that (3.42) follows by choosing $\varepsilon$ so small that $1-\varepsilon^{1 / 4} \geq 1 / 4$.

Finally, we turn to the proof of (3.43) and (3.44). In order to simplify the notation, let us write $\hat{V}$ instead of $f_{\sharp} V$. Using the same strategy as in [20, Proof of Proposition 5.7], we can estimate

$$
\left|\Phi_{\varepsilon} *\|\hat{V}\|(x)-\Phi_{\varepsilon} *\|V\|(x)\right| \leq I_{1}+I_{2},
$$

where

$$
I_{1}=\int\left|\Phi_{\varepsilon}(f(y)-x)-\Phi_{\varepsilon}(y-x)\right|\left|\Lambda_{n} \nabla f(y) \circ S\right| d V(y, S),
$$

and

$$
I_{2}=\int \Phi_{\varepsilon}(y-x) \| \Lambda_{n} \nabla f \circ S|-1| d V(y, S) .
$$

The first term can be estimated by observing that for some point $\hat{y}$ on the segment $[y-x, f(y)-x]$,

$$
\begin{aligned}
\left|\Phi_{\varepsilon}(f(y)-x)-\Phi_{\varepsilon}(y-x)\right| & \leq\left|\nabla \Phi_{\varepsilon}(\hat{y})\right||F(y)| \\
& \stackrel{(3.13)}{\leq}|F(y)|\left(\varepsilon^{-2}|\hat{y}| \Phi_{\varepsilon}(\hat{y})+c \chi_{B_{1} \backslash B_{1 / 2}}(\hat{y}) \exp \left(-\varepsilon^{-1}\right)\right) \\
& \stackrel{(3.45)}{\leq} c(n) \varepsilon^{\kappa-n-5} \chi_{B_{2}(x)}(y),
\end{aligned}
$$

and using that

$$
\left|\Lambda_{n} \nabla f(y) \circ S\right| \leq 1+\varepsilon^{\kappa-5}
$$

because of (3.49), so that

$$
I_{1} \leq \varepsilon^{\kappa-n-6}\|V\|\left(B_{2}(x)\right) .
$$

Concerning the second term in the sum, we can use (3.49) again to estimate

$$
I_{2} \leq c(n) \varepsilon^{-n-1} \varepsilon^{\kappa-5}\|V\|\left(B_{1}(x)\right) .
$$

Putting the two estimates together, we see that

$$
\left|\Phi_{\varepsilon} *\|\hat{V}\|(x)-\Phi_{\varepsilon} *\|V\|(x)\right| \leq \varepsilon^{\kappa-n-7}\|V\|\left(B_{2}(x)\right) .
$$


Analogous calculations lead to

$$
\left|\Phi_{\varepsilon} * \delta \hat{V}(x)-\Phi_{\varepsilon} * \delta V(x)\right| \leq \varepsilon^{\kappa-n-9}\|V\|\left(B_{2}(x)\right) .
$$

The rough estimates also give

$$
\left|\Phi_{\varepsilon} * \delta V(x)\right|,\left|\Phi_{\varepsilon} * \delta \hat{V}(x)\right| \leq \varepsilon^{-n-4}\|V\|\left(B_{2}(x)\right) .
$$

The estimates (3.54), (3.55), and (3.56) immediately yield

$$
\left|\frac{\Phi_{\varepsilon} * \delta \hat{V}}{\Phi_{\varepsilon} *\|\hat{V}\|+\varepsilon}-\frac{\Phi_{\varepsilon} * \delta V}{\Phi_{\varepsilon} *\|V\|+\varepsilon}\right| \leq \varepsilon^{\kappa-n-10}\|V\|\left(B_{2}(x)\right)+\varepsilon^{\kappa-2 n-13}\|V\|\left(B_{2}(x)\right)^{2},
$$

as well as

$$
\left|\frac{\left|\Phi_{\varepsilon} * \delta \hat{V}\right|^{2}}{\Phi_{\varepsilon} *\|\hat{V}\|+\varepsilon}-\frac{\left|\Phi_{\varepsilon} * \delta V\right|^{2}}{\Phi_{\varepsilon} *\|V\|+\varepsilon}\right| \leq \varepsilon^{\kappa-2 n-15}\|V\|\left(B_{2}(x)\right)^{2}+\varepsilon^{\kappa-3 n-17}\|V\|\left(B_{2}(x)\right)^{3} .
$$

Observe that, since spt $\|V\| \subset(U)_{1}$, the right-hand side of estimates (3.57) and (3.58) is zero whenever $\operatorname{dist}(x, \operatorname{clos}(U))>3$. Hence, (3.58) and the monotonicity of the mass $\|V\|\left(B_{2}(x)\right) \leq M$ imply that

$$
\begin{aligned}
& \left|\int_{\mathbb{R}^{n+1}} \eta_{j} \frac{\left|\Phi_{\varepsilon} * \delta V\right|^{2}}{\Phi_{\varepsilon} *\|V\|+\varepsilon} d x-\int_{\mathbb{R}^{n+1}} \eta_{j} \frac{\left|\Phi_{\varepsilon} * \delta\left(f_{\sharp} V\right)\right|^{2}}{\Phi_{\varepsilon} *\left\|f_{\sharp} V\right\|+\varepsilon} d x\right| \\
& \quad \leq\left(\varepsilon^{\kappa-2 n-15} M^{2}+\varepsilon^{\kappa-3 n-17} M^{3}\right) \int_{(U)_{3}} \eta_{j}(x) d x \leq \varepsilon^{\kappa-3 n-18}
\end{aligned}
$$

by possibly choosing a smaller value of $\varepsilon$ (depending on $U$ and $M$ ). This proves (3.44).

Finally, we prove (3.43). By (3.22), (3.57), and the properties of $\Phi_{\varepsilon}$, we deduce that

$$
\left\|\nabla^{l} h_{\varepsilon}(V)-\nabla^{l} h_{\varepsilon}(\hat{V})\right\| \leq \varepsilon^{\kappa-2 n-14-2 l}\left(M+M^{2}\right)
$$

for $l=0,1,2$. We can conclude using (3.59), (3.45)-(3.49) and suitable interpolations that:

$$
\begin{aligned}
& \left|\delta(V, \phi)\left(\eta_{j} h_{\varepsilon}(V)\right)-\delta(\hat{V}, \phi)\left(\eta_{j} h_{\varepsilon}(\hat{V})\right)\right| \\
& =\mid \int_{\mathbf{G}_{n}\left(\mathbb{R}^{n+1}\right)}\left\{\phi \nabla\left(\eta_{j} h_{\varepsilon}(V)\right) \cdot S+\eta_{j} h_{\varepsilon}(V) \cdot \nabla \phi\right\} d V(x, S) \\
& \quad-\int_{\mathbf{G}_{n}\left(\mathbb{R}^{n+1}\right)}\left\{\phi \circ f\left[\nabla\left(\eta_{j} h_{\varepsilon}(\hat{V})\right)\right] \circ f \cdot(\nabla f \circ S)\right. \\
& \left.\quad+\left(\eta_{j} h_{\varepsilon}(\hat{V})\right) \circ f \cdot(\nabla \phi \circ f)\right\}\left|\Lambda_{n} \nabla f \circ S\right| d V(x, S) \mid \\
& \leq \varepsilon^{\kappa-2 n-18} .
\end{aligned}
$$




\section{Existence of limit measures}

\subsection{The construction of the approximate flows}

Suppose $U$ and $\Gamma_{0}$ are as in Assumption 1.1. Together with the sets $D_{j}, K_{j}, \tilde{K}_{j}, \hat{K}_{j}$ introduced in Definition 3.14, for $k=0,1, \ldots$, we set

$$
D_{j, k}:=\left\{x \in U: \operatorname{dist}(x, \partial U) \geq \frac{1}{j^{1 / 4}}-k \exp \left(-j^{1 / 8}\right)\right\} .
$$

Once again, here the indices $j$ and $k$ are chosen in such a way that the corresponding sets $D_{j, k}$ are non-empty proper subsets of $U$. Observe that we have the elementary inclusions $D_{j, 0} \subset D_{j, k} \subset D_{j, k^{\prime}}$ for every $0 \leq k \leq k^{\prime}$, and that $D_{j} \subset D_{j, k}$ for every $k$.

Before proceeding with the construction of the time-discrete approximate flows, we need to introduce a suitable new class of test functions. Since $U$ is an open and bounded convex domain with boundary $\partial U$ of class $C^{2}$, there exists a neighborhood $(\partial U)_{s_{0}}$ such that, denoting $\mathrm{d}_{U}(x):=\operatorname{dist}\left(x, \mathbb{R}^{n+1} \backslash U\right)$ for $x \in(\partial U)_{s_{0}} \cap U$ the distance function from the boundary, the vector field $v_{U}(x):=-\nabla \mathrm{d}_{U}(x)$ is a $C^{1}$ extension to $(\partial U)_{s_{0}}^{-}:=(\partial U)_{s_{0}} \cap U$ of the exterior unit normal vector field to $\partial U$.

Definition 4.1 Define the tubular neighborhood of $\partial U$ and the vector field $v_{U}$ as above. Given an open set $W$, a function $\phi \in C^{1}\left(\mathbb{R}^{n+1} ; \mathbb{R}^{+}\right)$is said to be non decreasing in $W$ along the fibers of the normal bundle of $\partial U$ oriented by $\nu_{U}$, or simply $v_{U}$-non decreasing in $W$, if for every $x \in W \cap(\partial U)_{s_{0}}^{-}$the map

$$
t \mapsto \phi\left(x+t v_{U}(x)\right)
$$

is monotone non decreasing for $t$ such that $x+t v_{U}(x) \in W \cap(\partial U)_{s_{0}}^{-}$. For $j \in \mathbb{N}$, we will set

$$
\mathcal{R}_{j}:=\left\{\phi \in C^{1}\left(\mathbb{R}^{n+1} ; \mathbb{R}^{+}\right): \phi \text { is } v_{U} \text {-non decreasing in } \mathbb{R}^{n+1} \backslash D_{j}\right\} .
$$

The following proposition and its proof contain the constructive algorithm which produces the time-discrete approximations of our Brakke flow with fixed boundary.

Proposition 4.2 Let $U, \mathcal{E}_{0}=\left\{E_{0, i}\right\}_{i=1}^{N} \in \mathcal{O P}^{N}(U)$, and $\Gamma_{0}$ be as in Assumption 1.1. There exists a positive integer $J=J(n)$ with the following property. For every $j \geq J(n)$, there exist $\varepsilon_{j} \in(0,1)$ satisfying (3.31), $p_{j} \in \mathbb{N}$, and, for every $k \in\left\{0,1, \ldots, j 2^{p_{j}}\right\}$, a bounded open set $U_{j, k} \subset \mathbb{R}^{n+1}$ with boundary $\partial U_{j, k}$ of class $C^{2}$ and an open partition $\mathcal{E}_{j, k}=\left\{E_{j, k, i}\right\}_{i=1}^{N} \in$ $\mathcal{O P} \mathcal{P}^{N}\left(U_{j, k}\right)$ such that

$$
U_{j, 0}=U \text { and } \mathcal{E}_{j, 0}=\mathcal{E}_{0} \quad \text { for every } j,
$$

and such that, setting $\Delta t_{j}:=2^{-p_{j}}$, and defining $\Gamma_{j, k}:=U_{j, k} \backslash \bigcup_{i=1}^{N} E_{j, k, i}$, the following holds true:

(1) $\partial U_{j, k} \subset(\partial U)_{k} \exp \left(-j^{1 / 8}\right)$ and $U_{j, k} \Delta U \subset(\partial U)_{k} \exp \left(-j^{1 / 8}\right)$,

(2) $K_{j} \cap \Gamma_{j, k} \backslash D_{j, k} \subset\left(\Gamma_{0}\right)_{k} \exp \left(-j^{1 / 8}\right)$,

(3) $\Gamma_{j, k} \backslash K_{j} \subset\left(D_{j, k}\right)_{j-10}$.

Moreover, we have:

$$
\left\|\partial \mathcal{E}_{j, k}\right\|\left(\mathbb{R}^{n+1}\right) \leq\left\|\partial \mathcal{E}_{0}\right\|\left(\mathbb{R}^{n+1}\right)+k \Delta t_{j} \varepsilon_{j}^{1 / 6},
$$




$$
\begin{aligned}
& \frac{\left\|\partial \mathcal{E}_{j, k}\right\|\left(\mathbb{R}^{n+1}\right)-\left\|\partial \mathcal{E}_{j, k-1}\right\|\left(\mathbb{R}^{n+1}\right)}{\Delta t_{j}}+\frac{1}{4} \int_{\mathbb{R}^{n+1}} \eta_{j} \frac{\left|\Phi_{\varepsilon_{j}} * \delta\left(\partial \mathcal{E}_{j, k}\right)\right|^{2}}{\Phi_{\varepsilon_{j}} *\left\|\partial \mathcal{E}_{j, k}\right\|+\varepsilon_{j}} d x \\
& -\frac{\left(1-j^{-5}\right)}{\Delta t_{j}} \Delta_{j}\left\|\partial \mathcal{E}_{j, k-1}\right\|\left(D_{j}\right) \leq \varepsilon_{j}^{1 / 8} \\
& \frac{\left\|\partial \mathcal{E}_{j, k}\right\|(\phi)-\left\|\partial \mathcal{E}_{j, k-1}\right\|(\phi)}{\Delta t_{j}} \leq \delta\left(\partial \mathcal{E}_{j, k}, \phi\right)\left(\eta_{j} h_{\mathcal{E}_{j}}\left(\cdot, \partial \mathcal{E}_{j, k}\right)\right)+\varepsilon_{j}^{1 / 8}
\end{aligned}
$$

for every $k \in\left\{1, \ldots, j 2^{p_{j}}\right\}$ and $\phi \in \mathcal{A}_{j} \cap \mathcal{R}_{j}$.

Proof of Proposition 4.2 Set

$$
M:=\left\|\partial \mathcal{E}_{0}\right\|\left(\mathbb{R}^{n+1}\right)+1,
$$

let $\kappa=3 n+20$ as in Proposition 3.21, and consider the following set of conditions for $\varepsilon \in(0,1)$ :

$$
\left\{\begin{array}{l}
\varepsilon<\varepsilon_{*}:=\min \left\{\varepsilon_{1}, \ldots, \varepsilon_{5}\right\}, \text { with } \varepsilon_{*}=\varepsilon_{*}(n, U, M), \\
(3.31) \text { holds, namely } \varepsilon^{1 / 6} \leq 1 /(2 j), \\
2 \varepsilon^{\kappa-2} \leq j^{-10} \\
2 j \varepsilon^{-\kappa} \exp \left(-j^{1 / 8}\right) \leq 1 /\left(4 j^{1 / 4}\right) .
\end{array}\right.
$$

Notice that the conditions in (4.7) are compatible for large $j$, namely there exists $j_{0}$ with the property that for every $j \geq j_{0}$ the set of $\varepsilon \in(0,1)$ satisfying $(4.7)$ is not empty. Letting $J(n)$ be the number provided by Lemma 3.16, for every $j \geq \max \left\{j_{0}, J(n)\right\}$ we choose $\varepsilon_{j} \in(0,1)$ such that all conditions in (4.7) are met. Observe that $\lim _{j \rightarrow \infty} \varepsilon_{j}=0$. Then, we choose $p_{j} \in \mathbb{N}$ such that

$$
\Delta t_{j}:=\frac{1}{2^{p_{j}}} \in\left(2^{-1} \varepsilon_{j}^{\kappa}, \varepsilon_{j}^{\kappa}\right] .
$$

The argument is constructive, and it proceeds by means of an induction process on $k \in$ $\left\{0,1, \ldots, j 2^{p_{j}}\right\}$. We set $U_{j, 0}:=U$ and $\mathcal{E}_{j, 0}:=\mathcal{E}_{0}$. Properties (1), (2), (3), as well as the estimate in (4.3) are then trivially satisfied, given the definition of $M$ and since $U_{j, 0}=U$, $\Gamma_{0} \backslash D_{j, 0} \subset \Gamma_{0}$ and $\Gamma_{0} \backslash K_{j} \subset \Gamma_{0} \cap D_{j} \subset D_{j, 0}$. Next, let $k \geq 1$, and assume we obtained the open partition $\mathcal{E}_{j, k-1}=\left\{E_{j, k-1, i}\right\}_{i=1}^{N}$ of $U_{j, k-1}$ satisfying (1), (2), (3), and (4.3) with $k-1$ in place of $k$. We will now produce $U_{j, k}$ and $\mathcal{E}_{j, k}=\left\{E_{j, k, i}\right\}_{i=1}^{N}$ satisfying the same conditions with $k$. At the same time, we will also show that each inductive step satisfies (4.4) and (4.5). Before proceeding, let us record the inductive assumptions for $U_{j, k-1}$ and $\Gamma_{j, k-1}:=U_{j, k-1} \cap \cup_{i=1}^{N} \partial E_{j, k-1, i}$ in the following set of equations:

$$
\begin{aligned}
& \partial U_{j, k-1} \subset(\partial U)_{(k-1) \exp \left(-j^{1 / 8}\right)} \text { and } U_{j, k-1} \Delta U \subset(\partial U)_{(k-1) \exp \left(-j^{1 / 8}\right)}, \\
& K_{j} \cap \Gamma_{j, k-1} \backslash D_{j, k-1} \subset\left(\Gamma_{0}\right)_{(k-1) \exp \left(-j^{1 / 8}\right)}, \\
& \Gamma_{j, k-1} \backslash K_{j} \subset\left(D_{j, k-1}\right)_{j^{-10}}, \\
& \left\|\partial \mathcal{E}_{j, k-1}\right\|\left(\mathbb{R}^{n+1}\right) \leq\left\|\partial \mathcal{E}_{0}\right\|\left(\mathbb{R}^{n+1}\right)+(k-1) \Delta t_{j} \varepsilon_{j}^{1 / 6} .
\end{aligned}
$$

Step 1: area reducing Lipschitz deformation. First notice that $D_{j, k-1} \subset U_{j, k-1}$. Indeed, the definition of $D_{j, k-1},(4.9)$, and the choice of $\varepsilon_{j}$ imply that $D_{j, k-1} \cap\left(U_{j, k-1} \triangle U\right)=\emptyset$, so that our claim readily follows from $D_{j, k-1} \subset U$. In particular, $D_{j} \subset D_{j, k-1} \subset U_{j, k-1}$. Hence, we can choose $f_{1} \in \mathbf{E}\left(\mathcal{E}_{j, k-1}, D_{j}, j\right)$ such that, setting $\mathcal{E}_{j, k}^{\star}:=\left(f_{1}\right)_{\star} \mathcal{E}_{j, k-1}(\in$ $\mathcal{O} \mathcal{P}^{N}\left(U_{j, k-1}\right)$ by Lemma 3.5), we have 


$$
\left\|\partial \mathcal{E}_{j, k}^{\star}\right\|\left(\mathbb{R}^{n+1}\right)-\left\|\partial \mathcal{E}_{j, k-1}\right\|\left(\mathbb{R}^{n+1}\right) \leq\left(1-j^{-5}\right) \Delta_{j}\left\|\partial \mathcal{E}_{j, k-1}\right\|\left(D_{j}\right) .1
$$

Set $\Gamma_{j, k}^{\star}:=U_{j, k-1} \cap \bigcup_{i=1}^{N} \partial E_{j, k, i}^{\star}$, and note that

$$
\Gamma_{j, k}^{\star} \backslash D_{j}=\Gamma_{j, k-1} \backslash D_{j}
$$

and

$$
\left\|\partial \mathcal{E}_{j, k}^{\star}\right\|(\phi) \leq\left\|\partial \mathcal{E}_{j, k-1}\right\|(\phi) \quad \text { for every } \phi \in \mathcal{A}_{j} .
$$

Step 2: retraction. Outside of $D_{j, k-1}$, we perform a suitable retraction procedure so that $\Gamma_{j, k}^{\star} \backslash\left(D_{j, k-1} \cup K_{j}\right)$ is retracted to $\partial D_{j, k-1}$. This retraction step is not needed for $k=1$, since $\Gamma_{j, 1}^{\star} \cap D_{j, 0}^{c}=\Gamma_{j, 0} \cap D_{j, 0}^{c}$, and $\Gamma_{j, 0} \backslash K_{j} \subset D_{j, 0}$ already.

Define

$$
A_{j, k}:=\left\{x \in \partial\left(D_{j, k-1}\right)_{j^{-10}}: \operatorname{dist}\left(x, \Gamma_{0} \backslash D_{j}\right)>1 /\left(2 j^{1 / 4}\right)\right\},
$$

and observe that $\left.f_{1}\right|_{A_{j, k}}=\left.\mathrm{id}\right|_{A_{j, k}}$, so that $A_{j, k} \cap E_{j, k, i}^{\star}=A_{j, k} \cap \operatorname{int}\left(f_{1}\left(E_{j, k-1, i}\right)\right)=$ $A_{j, k} \cap E_{j, k-1, i}$ for every $i=1, \ldots, N$. In particular, $\Gamma_{j, k}^{\star} \cap A_{j, k}=\Gamma_{j, k-1} \cap A_{j, k}$.

We claim the validity of the following

Lemma 4.3 We have $A_{j, k} \cap \Gamma_{j, k}^{\star}=\emptyset$. Moreover, for any $x \in \partial A_{j, k}$ (the boundary as a subset of $\left.\partial\left(D_{j, k-1}\right)_{j-10}\right)$, we have dist $\left(x, \Gamma_{j, k}^{\star}\right) \geq j^{-10}$.

Proof By the discussion above, $A_{j, k} \cap \Gamma_{j, k}^{\star}=A_{j, k} \cap \Gamma_{j, k-1}$. By (4.11), $A_{j, k} \cap \Gamma_{j, k-1} \backslash$ $K_{j}=\emptyset$. If $x \in A_{j, k} \cap \Gamma_{j, k-1} \cap K_{j}$, then $x \in K_{j} \cap \Gamma_{j, k-1} \backslash D_{j, k-1}$. Then by (4.10), $\operatorname{dist}\left(x, \Gamma_{0}\right)<(k-1) \exp \left(-j^{1 / 8}\right) \leq 1 /\left(4 j^{1 / 4}\right)$, where the last inequality follows from $k \leq$ $j 2^{p_{j}} \leq 2 j \varepsilon_{j}^{-\kappa}$ and the choice of $\varepsilon_{j}$. By (4.16), we need to have some $\tilde{x} \in \Gamma_{0} \cap D_{j}$ such that $|x-\tilde{x}|<(k-1) \exp \left(-j^{1 / 8}\right)$. On the other hand, by the definitions of $D_{j, k-1}$ and $D_{j},|x-\tilde{x}| \geq$ $\operatorname{dist}\left(A_{j, k}, D_{j}\right)>1 / j^{1 / 4}$, and we have reached a contradiction. Thus the first claim follows. For the second claim, such point $x$ satisfies dist $\left(x, \Gamma_{0} \backslash D_{j}\right)=1 /\left(2 j^{1 / 4}\right)$. If there exists $\tilde{x} \in \Gamma_{j, k}^{\star}$ with $|x-\tilde{x}|<j^{-10}$, then $\tilde{x} \in \Gamma_{j, k-1}$, and dist $\left(\tilde{x}, \Gamma_{0} \backslash D_{j}\right)<1 /\left(2 j^{1 / 4}\right)+j^{-10}$, so that $\tilde{x} \in K_{j} \cap \Gamma_{j, k-1} \backslash D_{j, k-1}$. By (4.10), dist $\left(\tilde{x}, \Gamma_{0}\right) \leq(k-1) \exp \left(-j^{1 / 8}\right)$ and thus $\operatorname{dist}\left(x, \Gamma_{0}\right)<j^{-10}+(k-1) \exp \left(-j^{1 / 8}\right)$. Since dist $\left(x, \Gamma_{0} \backslash D_{j}\right)=1 /\left(2 j^{1 / 4}\right)$, this shows that there exists $\hat{x} \in \Gamma_{0} \cap D_{j}$ such that $|\hat{x}-x|<j^{-10}+(k-1) \exp \left(-j^{1 / 8}\right) \leq 1 /\left(2 j^{1 / 4}\right)$. On the other hand, dist $\left(\partial\left(D_{j, k-1}\right)_{j^{-10}}, D_{j}\right)>1 /\left(j^{1 / 4}\right)$, which is a contradiction. Thus we have the second claim.

Next, for each point $x \in \partial\left(D_{j, k-1}\right)_{j^{-10}}$, let $r_{0}(x) \in \partial D_{j, k-1}$ be the nearest point projection of $x$ onto $\partial D_{j, k-1}$, and set $r_{s}(x):=s x+(1-s) r_{0}(x)$ for $s \in(0,1)$. With this notation, define

$$
\operatorname{Ret}_{j, k}:=\left\{r_{s}(x): x \in A_{j, k}, s \in(0,1)\right\} .
$$

Lemma 4.4 We have $\left(D_{j, k-1}\right)_{j-10} \backslash\left(K_{j} \cup D_{j, k-1}\right) \subset \operatorname{Ret}_{j, k}$.

Proof For any point $\tilde{x} \in\left(D_{j, k-1}\right)_{j^{-10}} \backslash\left(K_{j} \cup D_{j, k-1}\right)$, there exist $s \in(0,1)$ and $x \in$ $\partial\left(D_{j, k-1}\right)_{j^{-10}}$ such that $\tilde{x}=r_{s}(x)$. The condition $\tilde{x} \notin K_{j}$ means that $\operatorname{dist}\left(\tilde{x}, \Gamma_{0} \backslash D_{j}\right) \geq$ $1 / j^{1 / 4}$, and then dist $\left(x, \Gamma_{0} \backslash D_{j}\right) \geq 1 / j^{1 / 4}-j^{-10}$. Thus $x \in A_{j, k}$ and $\tilde{x} \in \operatorname{Ret}_{j, k}$.

$\overline{{ }^{1} \text { Recall that } \Delta_{j}\left\|\partial \mathcal{E}_{j, k-1}\right\|\left(D_{j}\right)} \leq 0$ 
The set $A_{j, k}$ is a relatively open subset of $\partial\left(D_{j, k-1}\right)_{j^{-10}}$. Let $A_{j, k, l} \subset A_{j, k}$ be any of the (at most countably many) connected components of $A_{j, k}$ and define

$$
\operatorname{Ret}_{j, k, l}:=\left\{r_{s}(x): x \in A_{j, k, l}, s \in(0,1)\right\} .
$$

Lemma 4.5 We have $\left(A_{j, k, l} \cup\left(\partial A_{j, k, l}\right)_{j-10}\right) \cap \Gamma_{j, k}^{\star}=\emptyset$.

Proof The claim follows directly from Lemma 4.3.

Lemma 4.5 implies that for each $l$ there exists some $i(l) \in\{1, \ldots, N\}$ such that $E_{j, k, i(l)}^{\star}$ contains $A_{j, k, l} \cup\left(\partial A_{j, k, l}\right)_{j-10}$. For each index $l$, let $i(l)$ be this correspondence. We define for each $i=1, \ldots, N$

$$
\tilde{E}_{j, k, i}:=E_{j, k, i}^{\star} \cup\left(\cup_{i(l)=i} \operatorname{Ret}_{j, k, l}\right) .
$$

In other words, when $A_{j, k, l} \cup\left(\partial A_{j, k, l}\right)_{j^{-10}}$ is contained in $E_{j, k, i(l)}^{\star}$ with $i(l)=i$, then we replace the open partitions inside $\operatorname{Ret}_{j, k, l}$ by $\tilde{E}_{j, k, i}$. For the resulting open partition $\tilde{\mathcal{E}}_{j, k}:=\left\{\tilde{E}_{j, k, i}\right\}_{i=1}^{N} \in \mathcal{O} \mathcal{P}^{N}\left(U_{j, k-1}\right)$, define $\tilde{\Gamma}_{j, k}:=U_{j, k-1} \cap \cup_{i=1}^{N} \partial \tilde{E}_{j, k, i}$.

Lemma 4.6 We have

$$
\tilde{\Gamma}_{j, k} \backslash K_{j} \subset D_{j, k-1}
$$

and

$$
\tilde{\Gamma}_{j, k} \backslash D_{j, k-1}=\Gamma_{j, k}^{\star} \backslash\left(D_{j, k-1} \cup \operatorname{Ret}_{j, k}\right)=\Gamma_{j, k-1} \backslash\left(D_{j, k-1} \cup \operatorname{Ret}_{j, k}\right) .
$$

Proof Note that $\tilde{\Gamma}_{j, k} \cap \overline{\operatorname{Ret}_{j, k}} \backslash D_{j, k-1}=\emptyset$ since $\partial \operatorname{Ret}_{j, k} \backslash D_{j, k-1}$ is contained in some open partition by Lemma 4.5 and $\tilde{\Gamma}_{j, k} \cap \operatorname{Ret}_{j, k}=\emptyset$. If there exists $x \in \tilde{\Gamma}_{j, k} \backslash\left(K_{j} \cup D_{j, k-1}\right)$, then $x \notin \overline{\operatorname{Ret}_{j, k}}$ and thus $x \in \Gamma_{j, k}^{\star} \backslash\left(K_{j} \cup D_{j, k-1}\right)=\Gamma_{j, k-1} \backslash\left(K_{j} \cup D_{j, k-1}\right)$. By (4.11), $x \in\left(D_{j, k-1}\right)_{j^{-10}} \backslash\left(K_{j} \cup D_{j, k-1}\right)$. By Lemma 4.4, $x \in \operatorname{Ret}_{j, k}$, which is a contradiction. This proves the first claim. The second claim follows from the definition of $\tilde{\Gamma}_{j, k}$, in the sense that the new partition has no boundary in $\operatorname{Ret}_{j, k}$, while $\Gamma_{j, k}^{\star} \backslash\left(D_{j, k-1} \cup \operatorname{Ret}_{j, k}\right)$ is kept intact. The identity in (4.14) is also used to obtain the last equality.

Lemma 4.7 For any $\phi \in \mathcal{R}_{j}$ we have:

$$
\int_{\tilde{\Gamma}_{j, k}} \phi d \mathcal{H}^{n} \leq \int_{\Gamma_{j, k}^{\star}} \phi d \mathcal{H}^{n} .
$$

Proof Note that $\tilde{\Gamma}_{j, k} \Delta \Gamma_{j, k}^{\star} \subset\left(\partial D_{j, k-1} \cap \overline{\operatorname{Ret}_{j, k}}\right) \cup \operatorname{Ret}_{j, k}$, and that $\tilde{\Gamma}_{j, k} \cap \operatorname{Ret}_{j, k}=\emptyset$. Let $\operatorname{Ret}_{j, k, l}$ and $E_{j, k, i(l)}^{\star}$ be as before. For any $x \in \tilde{\Gamma}_{j, k} \cap \overline{\operatorname{Ret}_{j, k, l}} \subset \partial D_{j, k-1}$, consider $\tilde{x} \in \partial\left(D_{j, k-1}\right)_{j^{-10}}$ such that $r_{0}(\tilde{x})=x$. Note that $\tilde{x}=r_{1}(\tilde{x}) \in E_{j, k, i(l)}^{\star}$. If $r_{s}(\tilde{x}) \notin \Gamma_{j, k}^{\star}$ for all $s \in[0,1)$, then $r_{0}(\tilde{x})=x \in E_{j, k, i(l)}^{\star}$ and we have $x \in \tilde{E}_{j, k, i(l)}$, which is a contradiction to $x \in \tilde{\Gamma}_{j, k}$. Thus there exists $s \in[0,1)$ such that $r_{s}(\tilde{x}) \in \Gamma_{j, k}^{\star}$. In particular, we see that $\tilde{\Gamma}_{j, k} \cap \overline{\operatorname{Ret}_{j, k}}$ is in the image of $\Gamma_{j, k}^{\star} \cap \overline{\operatorname{Ret}_{j, k}}$ through the normal nearest point projection onto $\partial D_{j, k-1}$. Furthermore, since $r_{s}(\tilde{x})=x+s|\tilde{x}-x| v_{U}(x)$, and since $\phi$ is $v_{U}$-non decreasing in $\mathbb{R}^{n+1} \backslash D_{j}$, it holds $\phi(x) \leq \phi\left(r_{s}(\tilde{x})\right)$. Given that the normal nearest point projection onto $\partial D_{j, k-1}$ is a Lipschitz map with Lipschitz constant $=1$, the desired estimate follows from the area formula. 
Note that, as a corollary of Lemma 4.7, we have that, setting $\tilde{\mathcal{E}}_{j, k}=\left\{\tilde{E}_{j, k, i}\right\}_{i=1}^{N}$,

$$
\left\|\partial \tilde{\mathcal{E}}_{j, k}\right\|\left(\mathbb{R}^{n+1}\right) \leq\left\|\partial \mathcal{E}_{j, k}^{\star}\right\|\left(\mathbb{R}^{n+1}\right) .
$$

Step 3: motion by smoothed mean curvature with boundary damping. Let $\tilde{V}_{j, k}=$ $\partial \tilde{\mathcal{E}}_{j, k}$ as defined in (3.8), and compute $h_{\varepsilon_{j}}(\cdot):=h_{\varepsilon_{j}}\left(\cdot, \tilde{V}_{j, k}\right)$. Also, let $\eta_{j} \in \mathcal{A}_{j^{3 / 4}}$ be the cutoff function defined in Definition 3.15. Observe that $j$ has been chosen so that the conclusions of Lemma 3.16 hold. Define the smooth diffeomorphism $f_{j, k}(x):=x+\eta_{j}(x) h_{\varepsilon_{j}}(x) \Delta t_{j}$. Observe that the induction hypothesis (4.12), together with (4.15) and (4.20), implies that $\left\|\tilde{V}_{j, k}\right\|\left(\mathbb{R}^{n+1}\right) \leq M$ as defined in (4.6). Hence, by Lemma 3.16, and using (3.23) and the definition of $\Delta t_{j}$, we can conclude that $\left|\eta_{j} h_{\varepsilon} \Delta t_{j}\right| \leq \exp \left(-j^{1 / 8}\right)$ on $\tilde{K}_{j}$. By the choice of $\varepsilon_{j}$, we also have that $\left|\eta_{j} h_{\varepsilon} \Delta t_{j}\right| \leq j^{-10}$ everywhere.

Set $U_{j, k}:=f_{j, k}\left(U_{j, k-1}\right), E_{j, k, i}:=f_{j, k}\left(\tilde{E}_{j, k, i}\right)$ and $\Gamma_{j, k}:=U_{j, k} \cap \cup_{i=1}^{N} \partial E_{j, k, i}$.

Lemma 4.8 We have

$$
\partial U_{j, k} \subset(\partial U)_{k} \exp \left(-j^{1 / 8}\right) \quad \text { and } U_{j, k} \triangle U \subset(\partial U)_{k} \exp \left(-j^{1 / 8}\right),
$$

namely (4.9) with $k$ in place of $k-1$ holds true.

Proof Since $\left|x-f_{j, k}(x)\right| \leq \eta_{j}\left|h_{\varepsilon_{j}}\right| \Delta t_{j} \leq \exp \left(-j^{1 / 8}\right)$ on $K_{j}$ by Lemma 3.16(2), we see with (4.9) that $f_{j, k}\left(K_{j} \cap\left(\partial U_{j, k-1} \cup U_{j, k-1} \triangle U\right)\right) \subset(\partial U)_{k \exp \left(-j^{1 / 8}\right)}$. In order to show that also $f_{j, k}\left(\left(\partial U_{j, k-1} \cup U_{j, k-1} \triangle U\right) \backslash K_{j}\right) \subset(\partial U)_{k \exp \left(-j^{1 / 8}\right)}$, we next claim that

$$
\min \left\{\operatorname{dist}\left(\partial U_{j, k-1} \backslash K_{j}, \tilde{\Gamma}_{j, k}\right), \operatorname{dist}\left(\left(U_{j, k-1} \Delta U\right) \backslash K_{j}, \tilde{\Gamma}_{j, k}\right)\right\} \geq 1 /\left(4 j^{1 / 4}\right) .
$$

To see this, let $x \in\left(\partial U_{j, k-1} \cup\left(U_{j, k-1} \triangle U\right)\right) \backslash K_{j}$ and $y \in \tilde{\Gamma}_{j, k}$. Since $x \in \partial U_{j, k-1} \cup$ $\left(U_{j, k-1} \triangle U\right)$, by (4.9) there is $\tilde{x} \in \partial U$ such that $|x-\tilde{x}| \leq(k-1) \exp \left(-j^{1 / 8}\right)$. Now, if $y \notin K_{j}$, then by Lemma 4.6, $y \in D_{j, k-1}$. By the definition of $D_{j, k-1},|x-y| \geq|y-\tilde{x}|-|\tilde{x}-x| \geq$ $1 / j^{1 / 4}-2(k-1) \exp \left(-j^{1 / 8}\right)$, so that $|x-y| \geq 1 /\left(4 j^{1 / 4}\right)$. The same conclusion clearly holds if $y \in D_{j, k-1}$. Finally, if $y \in K_{j} \backslash D_{j, k-1}$ then, by (4.18), $y \in \Gamma_{j, k-1} \cap K_{j} \backslash D_{j, k-1}$. Then by (4.10), $y \in\left(\Gamma_{0}\right)_{(k-1)} \operatorname{exp(-j^{1/8})} \backslash D_{j, k-1}$. By the definition of $K_{j}$, we have $|x-y| \geq$ $j^{-1 / 4}-(k-1) \exp \left(-j^{1 / 8}\right)>1 /\left(4 j^{1 / 4}\right)$. This proves $(4.21)$. For any point $x \notin\left(\tilde{\Gamma}_{j, k}\right)_{1 / 4 j^{1 / 4}}$, note that

$$
\left|h_{\varepsilon_{j}}\left(x, \tilde{V}_{j, k}\right)\right| \leq \varepsilon_{j}^{-1} \int_{\tilde{\Gamma}_{j, k}}\left|\nabla \Phi_{\varepsilon_{j}}(x-y)\right| d \mathcal{H}^{n}(y) \leq M \exp \left(-1 / \varepsilon_{j}\right)<\exp \left(-j^{1 / 8}\right)
$$

for all sufficiently large $j$. This shows that $f_{j, k}\left(\left(\partial U_{j, k-1} \cup U_{j, k-1} \Delta U\right) \backslash K_{j}\right) \subset$ $(\partial U)_{k \exp \left(-j^{1 / 8}\right)}$ and concludes the proof.

Lemma 4.9 We have

$$
f_{j, k}\left(D_{j, k-1}\right) \cap\left(K_{j} \backslash D_{j, k}\right)=\emptyset .
$$

Proof Suppose, towards a contradiction, that $x \in f_{j, k}\left(D_{j, k-1}\right) \cap\left(K_{j} \backslash D_{j, k}\right)$. Since $\left|\Delta t_{j} \eta_{j} h_{\varepsilon_{j}}\right| \ll 1 / j^{1 / 4}$ for all points, $\hat{x}:=f_{j, k}^{-1}(x)$ is in $\tilde{K}_{j}$ in particular. Then, $\left|\eta_{j}(\hat{x}) h_{\varepsilon_{j}}(\hat{x}) \Delta t_{j}\right| \leq \exp \left(-j^{1 / 8}\right)$. This means that $|x-\hat{x}| \leq \exp \left(-j^{1 / 8}\right)$. Since $x \notin D_{j, k}$, we need to have $\hat{x} \notin D_{j, k-1}$ by the definition of these sets. But this is a contradiction since $x=f_{j, k}(\hat{x}) \in f_{j, k}\left(D_{j, k-1}\right)$ and $f_{j, k}$ is bijective. 
Lemma 4.10 We have

$$
\left(\Gamma_{j, k} \cap K_{j}\right) \backslash D_{j, k} \subset\left(\Gamma_{0}\right)_{k \exp \left(-j^{1 / 8}\right)},
$$

namely (4.10) with $k$ in place of $k-1$ holds true.

Proof For any $x \in\left(\Gamma_{j, k} \cap K_{j}\right) \backslash D_{j, k}$, by Lemma 4.9, $x \notin f_{j, k}\left(D_{j, k-1}\right)$ and there exists $\hat{x} \in \tilde{\Gamma}_{j, k} \backslash D_{j, k-1}$ such that $f_{j, k}(\hat{x})=x$. By (4.17) and (4.18), $\hat{x} \in\left(\Gamma_{j, k}^{\star} \cap K_{j}\right) \backslash D_{j, k-1}=$ $\left(\Gamma_{j, k-1} \cap K_{j}\right) \backslash D_{j, k-1}$. By (4.10), $\hat{x} \in\left(\Gamma_{0}\right)_{(k-1) \exp \left(-j^{1 / 8}\right)}$; on the other hand, $\hat{x} \in K_{j}$ implies $|x-\hat{x}| \leq \exp \left(-j^{1 / 8}\right)$. These two estimates together prove (4.22).

Lemma 4.11 We have

$$
\Gamma_{j, k} \backslash K_{j} \subset\left(D_{j, k}\right)_{j-10},
$$

namely (4.11) with $k$ in place of $k-1$ holds true.

Proof If $x \in \Gamma_{j, k} \backslash K_{j}$, then there is $\tilde{x} \in \tilde{\Gamma}_{j, k}$ such that $x=f_{j, k}(\tilde{x})$. If $\tilde{x} \notin K_{j}$, then $x \in D_{j, k-1} \subset D_{j, k}$ by Lemma 4.6, and since $|x-\tilde{x}|<j^{-10}$ by the properties of the diffeomorphism $f_{j, k}$ our claim holds true. Hence, suppose that $\tilde{x} \in K_{j}$. Since in this case $|x-\tilde{x}| \leq \exp \left(-j^{1 / 8}\right)$, if $\tilde{x} \in D_{j, k-1}$ then evidently $x \in D_{j, k}$, and the proof is complete. On the other hand, we claim that it has to be $\tilde{x} \in D_{j, k-1}$. Indeed, otherwise we would have $\tilde{x} \in \tilde{\Gamma}_{j, k} \cap K_{j} \backslash D_{j, k-1}$, and thus, again by Lemma 4.6, $\tilde{x} \in \Gamma_{j, k}^{\star} \cap K_{j} \backslash D_{j, k-1}=\Gamma_{j, k-1} \cap$ $K_{j} \backslash D_{j, k-1}$. But then, by (4.10), there exists $y \in \Gamma_{0}$ such that $|\tilde{x}-y|<(k-1) \exp \left(-j^{1 / 8}\right)$. Since $\tilde{x} \notin D_{j, k-1}$, we have $y \notin D_{j}$, and therefore $\operatorname{dist}\left(x,\left(\Gamma_{0} \backslash D_{j}\right)\right) \leq|x-\tilde{x}|+|\tilde{x}-y|<$ $k \exp \left(-j^{1 / 8}\right)<1 / j^{1 / 4}$. But this contradicts the fact that $x \notin K_{j}$ and completes the proof.

Conclusion. Together, Lemmas 4.8, 4.10 and 4.11 complete the induction step from $k-1$ to $k$ for properties (1), (2), (3). Concerning (4.3), first we observe that, since $f_{j, k}$ is a diffeomorphism,

$$
\begin{aligned}
& \partial \mathcal{E}_{j, k}=\operatorname{var}\left(\bigcup_{i=1}^{N}\left(U_{j, k} \cap \partial E_{j, k, i}\right), 1\right) \\
& =\operatorname{var}\left(f_{j, k}\left(\bigcup_{i=1}^{N}\left(U_{j, k-1} \cap \partial \tilde{E}_{j, k, i}\right)\right), 1\right)=\left(f_{j, k}\right)_{\sharp} \partial \tilde{\mathcal{E}}_{j, k} .
\end{aligned}
$$

We can then use (3.42) with $V=\partial \tilde{\mathcal{E}}_{j, k}, M$ as defined in (4.6), $\varepsilon=\varepsilon_{j}$, and $\Delta t=\Delta t_{j}$ in order to conclude that

$$
\left\|\partial \mathcal{E}_{j, k}\right\|\left(\mathbb{R}^{n+1}\right) \leq 2 \Delta t_{j} \varepsilon_{j}^{1 / 4}+\left\|\partial \tilde{\mathcal{E}}_{j, k}\right\|\left(\mathbb{R}^{n+1}\right) .
$$

Combining (4.25) with (4.15) and (4.20), and using that $2 \varepsilon_{j}^{1 / 4}<\varepsilon_{j}^{1 / 6}$, we get

$$
\left\|\partial \mathcal{E}_{j, k}\right\|\left(\mathbb{R}^{n+1}\right) \leq\left\|\partial \mathcal{E}_{j, k-1}\right\|\left(\mathbb{R}^{n+1}\right)+\Delta t_{j} \varepsilon_{j}^{1 / 6},
$$

which, together with (4.12), gives (4.3). Last, we show that the construction of the induction step satisfies (4.4) and (4.5). Since $\varepsilon_{j}$ satisfies (3.31) and (4.3) implies $\left\|\left(f_{j, k}\right)_{\sharp} \partial \tilde{\mathcal{E}}_{j, k}\right\|\left(\mathbb{R}^{n+1}\right) \leq M$, so that the estimates in (3.43) and (3.44) hold true. Then (4.4) follows from (3.42), (3.44), (4.20) and (4.13). Finally, (4.5) is a consequence of (3.41), (3.43), (4.19) and (4.15). 
We are now in a position to define an approximate flow of open partitions. As anticipated in the introduction, the flow is piecewise constant in time; the parameter $\Delta t_{j}$ defined in (4.8) is the epoch length, namely the length of the time intervals in which the flow is set to be constant.

Definition 4.12 For every $j \geq \max \left\{j_{0}, J(n)\right\}$, define a family $\mathcal{E}_{j}(t)$ for $t \in[0, j]$ by setting

$$
\mathcal{E}_{j}(t):=\mathcal{E}_{j, k} \quad \text { if } t \in\left((k-1) \Delta t_{j}, k \Delta t_{j}\right] .
$$

\subsection{Convergence in the sense of measures}

Proposition 4.13 Under the assumptions of Proposition 4.2, there exist a subsequence $\left\{j_{\ell}\right\}_{\ell=1}^{\infty}$ and a one-parameter family of Radon measures $\left\{\mu_{t}\right\}_{t \geq 0}$ on $U$ such that

$$
\mu_{t}(\phi)=\lim _{\ell \rightarrow \infty}\left\|\partial \mathcal{E}_{j_{\ell}}(t)\right\|(\phi)
$$

for all $\phi \in C_{c}(U)$ and $t \in \mathbb{R}^{+}$. The limits $\lim _{s \rightarrow t+} \mu_{s}(\phi)$ and $\lim _{s \rightarrow t-} \mu_{s}(\phi)$ exist and satisfy

$$
\lim _{s \rightarrow t+} \mu_{s}(\phi) \leq \mu_{t}(\phi) \leq \lim _{s \rightarrow t-} \mu_{s}(\phi)
$$

for all $\phi \in C_{c}\left(U ; \mathbb{R}^{+}\right)$and $t \in \mathbb{R}^{+}$. Furthermore, $\lim _{s \rightarrow t+} \mu_{s}(\phi)=\lim _{s \rightarrow t-} \mu_{s}(\phi)$ for all $t \in \mathbb{R}^{+} \backslash B$, where $B \subset \mathbb{R}^{+}$is countable. Finally, for every $T>0$ we have

$$
\limsup _{\ell \rightarrow \infty} \int_{0}^{T}\left(\int_{\mathbb{R}^{n+1}} \eta_{j_{\ell}} \frac{\left|\Phi_{\varepsilon_{j_{\ell}}} * \delta\left(\partial \mathcal{E}_{j_{\ell}}(t)\right)\right|^{2}}{\Phi_{\varepsilon_{j_{\ell}}} *\left\|\partial \mathcal{E}_{j_{\ell}}(t)\right\|+\varepsilon_{j_{\ell}}} d x-\frac{1}{\Delta t_{j_{\ell}}} \Delta_{j_{\ell}}\left\|\partial \mathcal{E}_{j_{\ell}}(t)\right\|\left(D_{j_{\ell}}\right)\right) d t<\infty,
$$

and for a.e. $t \in \mathbb{R}^{+}$it holds

$$
\lim _{\ell \rightarrow \infty} j_{\ell}^{2(n+1)} \Delta_{j_{\ell}}\left\|\partial \mathcal{E}_{j_{\ell}}(t)\right\|\left(D_{j_{\ell}}\right)=0 .
$$

Proof Let $2_{\mathbb{Q}}$ be the set of all non-negative numbers of the form $\frac{i}{2^{j}}$ for some $i, j \in \mathbb{N} \cup\{0\}$. $2_{\mathbb{Q}}$ is countable and dense in $\mathbb{R}^{+}$. For each fixed $T \in \mathbb{N}$, the mass estimate in (4.3) implies that

$$
\limsup _{j \rightarrow \infty} \sup _{t \in[0, T]}\left\|\partial \mathcal{E}_{j}(t)\right\|\left(\mathbb{R}^{n+1}\right) \leq\left\|\partial \mathcal{E}_{0}\right\|\left(\mathbb{R}^{n+1}\right) .
$$

Therefore, by a diagonal argument we can choose a subsequence $\left\{j_{\ell}\right\}$ and a family of Radon measures $\left\{\mu_{t}\right\}_{t \in 2_{\mathbb{Q}}}$ on $\mathbb{R}^{n+1}$ such that

$$
\mu_{t}(\phi)=\lim _{\ell \rightarrow \infty}\left\|\partial \mathcal{E}_{j_{\ell}}(t)\right\|(\phi) \quad \text { for every } \phi \in C_{c}\left(\mathbb{R}^{n+1}\right), \text { for every } t \in 2 \mathbb{Q} .
$$

Furthermore, with (4.31), we also deduce that

$$
\mu_{t}\left(\mathbb{R}^{n+1}\right) \leq\left\|\partial \mathcal{E}_{0}\right\|\left(\mathbb{R}^{n+1}\right) \quad \text { for every } t \in 2_{\mathbb{Q}} .
$$

Next, let $Z:=\left\{\phi_{q}\right\}_{q \in \mathbb{N}}$ be a countable subset of $C_{c}^{2}\left(U ; \mathbb{R}^{+}\right)$which is dense in $C_{c}\left(U ; \mathbb{R}^{+}\right)$ with respect to the supremum norm. We claim that the function

$$
t \in 2_{\mathbb{Q}} \mapsto g_{q}(t):=\mu_{t}\left(\phi_{q}\right)-t\left\|\nabla^{2} \phi_{q}\right\|_{\infty}\left\|\partial \mathcal{E}_{0}\right\|\left(\mathbb{R}^{n+1}\right)
$$


is monotone non-increasing. To see this, first observe that since $\phi_{q}$ has compact support, and since the definition in (4.34) depends linearly on $\phi_{q}$, we can assume without loss of generality that $\phi_{q}<1$. For convenience, for $t \leq 0$, we define $g_{q}(t):=\mu_{0}\left(\phi_{q}\right)=\left\|\partial \mathcal{E}_{0}\right\|\left(\phi_{q}\right)$. Next, given any $j \geq J(n)$ as in Proposition 4.2, for every positive function $\phi$ such that $\eta_{j} \phi \in \mathcal{A}_{j}$ we can compute

$$
\begin{aligned}
& \delta\left(\partial \mathcal{E}_{j}(t), \phi\right)\left(\eta_{j} h_{\varepsilon_{j}}\right)=\delta\left(\partial \mathcal{E}_{j}(t)\right)\left(\eta_{j} \phi h_{\varepsilon_{j}}\right) \\
& +\int_{\mathbf{G}_{n}\left(\mathbb{R}^{n+1}\right)} \eta_{j}(x) h_{\varepsilon_{j}} \cdot S^{\perp}(\nabla \phi(x)) d\left(\partial \mathcal{E}_{j}(t)\right)(x, S) \\
& =: I_{1}+I_{2}
\end{aligned}
$$

for every $t \in[0, j]$, and where $h_{\varepsilon_{j}}(\cdot)=h_{\varepsilon_{j}}\left(\cdot, \partial \mathcal{E}_{j}(t)\right)$. By the choice of $\varepsilon_{j}$, and since $\eta_{j} \phi \in \mathcal{A}_{j}$, we can use (3.33) to estimate

$$
I_{1} \leq \varepsilon_{j}^{1 / 4}-\left(1-\varepsilon_{j}^{1 / 4}\right) \int_{\mathbb{R}^{n+1}} \eta_{j} \phi \frac{\left|\Phi_{\varepsilon_{j}} * \delta\left(\partial \mathcal{E}_{j}(t)\right)\right|^{2}}{\Phi_{\varepsilon_{j}} *\left\|\partial \mathcal{E}_{j}(t)\right\|+\varepsilon_{j}} d x,
$$

whereas Young's inequality together with (3.34) yields

$$
\begin{aligned}
I_{2} & \leq \frac{1}{2} \int_{\mathbb{R}^{n+1}} \eta_{j} \phi\left|h_{\varepsilon_{j}}\right|^{2} d\left\|\partial \mathcal{E}_{j}(t)\right\|+\frac{1}{2} \int_{\mathbb{R}^{n+1}} \eta_{j} \frac{\left|S^{\perp}(\nabla \phi)\right|^{2}}{\phi} d\left\|\partial \mathcal{E}_{j}(t)\right\| \\
& \leq \frac{\varepsilon_{j}^{1 / 4}}{2}+\left(\frac{1}{2}+\frac{\varepsilon_{j}^{1 / 4}}{2}\right) \int_{\mathbb{R}^{n+1}} \eta_{j} \phi \frac{\left|\Phi_{\varepsilon_{j}} * \delta\left(\partial \mathcal{E}_{j}(t)\right)\right|^{2}}{\Phi_{\varepsilon_{j}} *\left\|\partial \mathcal{E}_{j}(t)\right\|+\varepsilon_{j}} d x \\
& +\frac{1}{2} \int_{\mathbb{R}^{n+1}} \eta_{j} \frac{\left|S^{\perp}(\nabla \phi)\right|^{2}}{\phi} d\left\|\partial \mathcal{E}_{j}(t)\right\| .
\end{aligned}
$$

Plugging (4.36) and (4.37) into (4.35), we obtain

$$
\delta\left(\partial \mathcal{E}_{j}(t), \phi\right)\left(\eta_{j} h_{\varepsilon_{j}}\right) \leq 2 \varepsilon_{j}^{\frac{1}{4}}+\frac{1}{2} \int_{\mathbb{R}^{n+1}} \eta_{j} \frac{|\nabla \phi|^{2}}{\phi} d\left\|\partial \mathcal{E}_{j}(t)\right\|
$$

for every $t \in[0, j]$ and for every positive function $\phi$ such that $\eta_{j} \phi \in \mathcal{A}_{j}$. Now, for every $T \in \mathbb{N}$, for every $\phi_{q} \in Z$ with $\phi_{q}<1$, and for every sufficiently large $i \in \mathbb{N}$, choose $j_{*} \geq \max \{T, J(n)\}$ so that

(i) $\phi_{q}+i^{-1} \in \mathcal{A}_{j} \cap \mathcal{R}_{j}$,

(ii) $\eta_{j}\left(\phi_{q}+i^{-1}\right) \in \mathcal{A}_{j}$

for every $j \geq j_{*}$. Using that $\eta_{j} \in \mathcal{A}_{j^{3 / 4}}$ for every $j \geq J(n)$ and that $\phi_{q}=0$ outside some compact set $K \subset U$, it is easily seen that the two conditions above can be met by choosing $j_{*}$ sufficiently large, depending on $i,\left\|\phi_{q}\right\|_{C^{2}}$, and $K$. In particular, $j_{*}$ is so large that $\phi_{q} \equiv 0$ on $(\partial U)_{s_{0}}^{-} \backslash D_{j_{*}}$, so that $\phi_{q}+i^{-1}$ is trivially $v_{U}$-non decreasing in $\mathbb{R}^{n+1} \backslash D_{j_{*}}$ because it is constant in there. For any fixed $t_{1}, t_{2} \in[0, T] \cap 2_{\mathbb{Q}}$ with $t_{2}>t_{1}$, choose a larger $j_{*}$, so that both $t_{1}$ and $t_{2}$ are integer multiples of $1 / 2^{p_{j_{*}}}$. Then, both $t_{2}$ and $t_{1}$ are integer multiples of $\Delta t_{j_{\ell}}$ for every $j_{\ell} \geq j_{*}$. Hence, for every $j_{\ell} \geq j_{*}$ we can apply (4.5) repeatedly with $\phi=\phi_{q}+i^{-1} \in \mathcal{A}_{j_{\ell}} \cap \mathcal{R}_{j_{\ell}}$ and (4.38) again with $\bar{\phi}=\phi_{q}+i^{-1}$ so that $\eta_{j_{\ell}} \phi \in \mathcal{A}_{j_{\ell}}$ in order to deduce

$$
\begin{aligned}
& \left\|\partial \mathcal{E}_{j_{\ell}}\left(t_{2}\right)\right\|\left(\phi_{q}+i^{-1}\right)-\left\|\partial \mathcal{E}_{j_{\ell}}\left(t_{1}\right)\right\|\left(\phi_{q}+i^{-1}\right) \\
& \quad \leq\left(\varepsilon_{j_{\ell}}^{1 / 8}+2 \varepsilon_{j_{\ell}}^{1 / 4}\right)\left(t_{2}-t_{1}\right)+\frac{1}{2} \int_{t_{1}}^{t_{2}} \int_{\mathbb{R}^{n+1}} \eta_{j_{\ell}} \frac{\left|\nabla \phi_{q}\right|^{2}}{\phi_{q}+i^{-1}} d\left\|\partial \mathcal{E}_{j_{\ell}}(t)\right\| d t .
\end{aligned}
$$


As we let $\ell \rightarrow \infty$, the left-hand side of (4.39) can be bounded from below, using (4.31) and (4.32), as follows:

$$
\geq \mu_{t_{2}}\left(\phi_{q}\right)-\mu_{t_{1}}\left(\phi_{q}\right)-i^{-1}\left\|\partial \mathcal{E}_{0}\right\|\left(\mathbb{R}^{n+1}\right) .
$$

In order to estimate the right-hand side of (4.39), we note that

$$
\frac{\left|\nabla \phi_{q}\right|^{2}}{\phi_{q}+i^{-1}} \leq \frac{\left|\nabla \phi_{q}\right|^{2}}{\phi_{q}} \leq 2\left\|\nabla^{2} \phi_{q}\right\|_{\infty},
$$

so that if we plug (4.41) in (4.39), use that $\eta_{j_{\ell}} \leq 1$, let $\ell \rightarrow \infty$ by means of (4.31), and finally let $i \rightarrow \infty$ we conclude

$$
\mu_{t_{2}}\left(\phi_{q}\right)-\mu_{t_{1}}\left(\phi_{q}\right) \leq\left\|\nabla^{2} \phi_{q}\right\|_{\infty}\left\|\partial \mathcal{E}_{0}\right\|\left(\mathbb{R}^{n+1}\right)\left(t_{2}-t_{1}\right)
$$

for every $t_{1}, t_{2} \in[0, T] \cap 2 \mathbb{Q}$ with $t_{2}>t_{1}$ and for any $\phi_{q} \in Z$ with $\phi_{q}<1$, thus proving that the function defined in (4.34) is indeed monotone non-increasing on [0,T]. Since $T$ is arbitrary, the same holds on $\mathbb{R}^{+}$.

Define now

$$
B:=\left\{t \in \mathbb{R}^{+}: \lim _{2_{\mathbb{Q}} \ni s \rightarrow t-} g_{q}(s)>\lim _{2_{\mathbb{Q}} \ni s \rightarrow t+} g_{q}(s) \text { for some } q \in \mathbb{N}\right\} .
$$

By the monotonicity of each $g_{q}, B$ is a countable subset of $\mathbb{R}^{+}$, and for every $t \in \mathbb{R}^{+} \backslash\left(B \cup 2_{\mathbb{Q}}\right)$ we can define $\mu_{t}\left(\phi_{q}\right)$ for every $\phi_{q} \in Z$ by

$$
\mu_{t}\left(\phi_{q}\right):=\lim _{2 \mathbb{Q} \ni s \rightarrow t}\left(g_{q}(s)+s\left\|\nabla^{2} \phi_{q}\right\|_{\infty}\left\|\partial \mathcal{E}_{0}\right\|\left(\mathbb{R}^{n+1}\right)\right)=\lim _{2 \mathbb{Q} \ni s \rightarrow t} \mu_{s}\left(\phi_{q}\right) .
$$

We claim that

$$
\exists \lim _{\ell \rightarrow \infty}\left\|\partial \mathcal{E}_{j_{\ell}}(t)\right\|\left(\phi_{q}\right)=\mu_{t}\left(\phi_{q}\right) \quad \text { for every } t \in \mathbb{R}^{+} \backslash\left(B \cup 2_{\mathbb{Q}}\right) \text { and } \phi_{q} \in Z .
$$

Indeed, due to the definition of $\partial \mathcal{E}_{j_{\ell}}(t)$, there exists a sequence $\left\{t_{\ell}\right\}_{\ell=1}^{\infty} \subset 2 \mathbb{Q}$ with $t_{\ell}>t$ such that $\lim _{\ell \rightarrow \infty} t_{\ell}=t$ and $\partial \mathcal{E}_{j_{\ell}}(t)=\partial \mathcal{E}_{j_{\ell}}\left(t_{\ell}\right)$. For any $s \in 2_{\mathbb{Q}}$ with $s>t$, and for all suffciently large $\ell$ so that $s>t_{\ell}$, we deduce from (4.39) that

$$
\left\|\partial \mathcal{E}_{j_{\ell}}(s)\right\|\left(\phi_{q}+i^{-1}\right) \leq\left\|\partial \mathcal{E}_{j_{\ell}}\left(t_{\ell}\right)\right\|\left(\phi_{q}+i^{-1}\right)+\mathrm{O}(s-t) .
$$

Taking the $\lim \inf _{\ell \rightarrow \infty}$ and then the $\lim _{i \rightarrow \infty}$ on both sides of (4.45) we obtain that

$$
\mu_{s}\left(\phi_{q}\right) \leq \liminf _{\ell \rightarrow \infty}\left\|\partial \mathcal{E}_{j_{\ell}}\left(t_{\ell}\right)\right\|\left(\phi_{q}\right)+\mathrm{O}(s-t),
$$

so that when we let $s \rightarrow t+$ the definition of $\mu_{t}$ and the fact that $\partial \mathcal{E}_{j_{\ell}}\left(t_{\ell}\right)=\partial \mathcal{E}_{j_{\ell}}(t)$ yield

$$
\mu_{t}\left(\phi_{q}\right) \leq \liminf _{\ell \rightarrow \infty}\left\|\partial \mathcal{E}_{j_{\ell}}(t)\right\|\left(\phi_{q}\right) .
$$

An analogous argument provides, at the same time,

$$
\limsup _{\ell \rightarrow \infty}\left\|\partial \mathcal{E}_{j \ell}(t)\right\|\left(\phi_{q}\right) \leq \mu_{t}\left(\phi_{q}\right),
$$

so that (4.47) and (4.48) together complete the proof of (4.44). Since $Z$ is dense in $C_{c}\left(U ; \mathbb{R}^{+}\right)$, (4.44) determines the limit measure uniquely, and the convergence holds for every $\phi \in C_{c}(U)$ at every $t \in \mathbb{R}^{+} \backslash B$. On the other hand, since $B$ is countable we can extract a further subsequence of $\left\{\partial \mathcal{E}_{j_{\ell}}(t)\right\}_{\ell=1}^{\infty}$ converging to a Radon measure $\mu_{t}$ in $U$ for every $t \geq 0$. The continuity of $\mu_{t}(\phi)$ on $\mathbb{R}^{+} \backslash B$ follows from the definition of $B$ and a density argument. The 
existence of limits and the inequalities (4.28) can be also deduced from (4.42) in the case $\phi=\phi_{q}$, and by density for $\phi \in C_{c}\left(U ; \mathbb{R}^{+}\right)$. This completes the proof of the first part of the statement.

The claim in (4.29) follows from (4.4). Finally, (4.29) implies that for each $T>0$

$$
\lim _{\ell \rightarrow \infty} \int_{0}^{T}-j^{2(n+1)} \Delta_{j_{\ell}}\left\|\partial \mathcal{E}_{j_{\ell}}(t)\right\|\left(D_{j_{\ell}}\right) d t \lesssim \lim _{\ell \rightarrow \infty} j_{\ell}^{2(n+1)} \Delta t_{j_{\ell}}=0,
$$

where in the last identity we have used that

$$
\Delta t_{j_{\ell}} \leq \varepsilon_{j_{\ell}}^{\kappa} \ll j_{\ell}^{-2(n+1)},
$$

given the definition of $\kappa$ and the fact that $\varepsilon_{j}$ satisfies (3.31). The proof is now complete.

\section{Brakke's inequality, rectifiability and integrality of the limit}

In the next proposition we deduce further information concerning the family $\left\{\mu_{t}\right\}_{t \geq 0}$ of measures in $U$ introduced in Proposition 4.13.

Proposition 5.1 Let $\left\{\partial \mathcal{E}_{j_{\ell}}(t)\right\}$ for $\ell \in \mathbb{N}$ and $t \geq 0$, and $\left\{\mu_{t}\right\}$ for $t \geq 0$ be as in Proposition 4.13 satisfying (4.27), (4.29) and (4.30). Then, we have the following.

(1) For a.e. $t \in \mathbb{R}^{+}$the measure $\mu_{t}$ is integral, namely there exists an integral varifold $V_{t} \in \mathbf{I V}_{n}(U)$ such that $\mu_{t}=\left\|V_{t}\right\|$.

(2) For a.e. $t \in \mathbb{R}^{+}$, if a subsequence $\left\{j_{\ell}^{\prime}\right\}_{\ell=1}^{\infty} \subset\left\{j_{\ell}\right\}_{\ell=1}^{\infty}$ is such that

$$
\sup _{\ell \in \mathbb{N}} \int_{\mathbb{R}^{n+1}} \eta_{j_{\ell}^{\prime}} \frac{\left|\Phi_{\varepsilon_{j_{\ell}^{\prime}}} * \delta\left(\partial \mathcal{E}_{j_{\ell}^{\prime}}(t)\right)\right|^{2}}{\Phi_{\varepsilon_{j_{\ell}^{\prime}}} *\left\|\partial \mathcal{E}_{j_{\ell}^{\prime}}(t)\right\|+\varepsilon_{j_{\ell}^{\prime}}} d x<\infty,
$$

then $\partial \mathcal{E}_{j_{\ell}^{\prime}}(t)$ converges to $V_{t} \in \mathbf{I V}_{n}(U)$ as varifolds in $U$ as $\ell \rightarrow \infty$, namely

$$
\lim _{\ell \rightarrow \infty} \partial \mathcal{E}_{j_{\ell}^{\prime}}(t)(\varphi)=V_{t}(\varphi) \quad \text { for every } \varphi \in C_{c}\left(\mathbf{G}_{n}(U)\right) .
$$

(3) For a.e. $t \in \mathbb{R}^{+}, V_{t}$ has generalized mean curvature $h\left(\cdot, V_{t}\right)$ in $U$ which satisfies

$$
\int_{U}\left|h\left(\cdot, V_{t}\right)\right|^{2} \phi d\left\|V_{t}\right\| \leq \liminf _{\ell \rightarrow \infty} \int_{\mathbb{R}^{n+1}} \phi \eta_{j_{\ell}} \frac{\left|\Phi_{\varepsilon_{j_{\ell}}} * \delta\left(\partial \mathcal{E}_{j_{\ell}}(t)\right)\right|^{2}}{\Phi_{\varepsilon_{j_{\ell}}} *\left\|\partial \mathcal{E}_{j_{\ell}}(t)\right\|+\varepsilon_{j_{\ell}}} d x<\infty
$$

for any $\phi \in C_{c}\left(U ; \mathbb{R}^{+}\right)$.

Before proving Proposition 5.1, we need to state two important results, which are obtained by suitably modifying [20, Theorem $7.3 \&$ Theorem 8.6], respectively.

Theorem 5.2 (Rectifiability Theorem). Suppose that $\left\{U_{j_{\ell}}\right\}_{\ell=1}^{\infty}$ are open sets in $\mathbb{R}^{n+1},\left\{\mathcal{E}_{j_{\ell}}\right\}_{\ell=1}^{\infty}$ are such that $\mathcal{E}_{j_{\ell}} \in \mathcal{O} \mathcal{P}^{N}\left(U_{j_{\ell}}\right)$, and $\left\{\varepsilon_{j_{\ell}}\right\}_{l=1}^{\infty} \subset(0,1)$. Suppose that they satisfy

(1) $\partial U_{j_{\ell}} \subset(\partial U)_{1 /\left(4 j_{\ell}^{1 / 4}\right)}$ and $U_{j_{\ell}} \Delta U \subset(\partial U)_{1 /\left(4 j_{\ell}^{1 / 4}\right)}$,

(2) $\lim _{\ell \rightarrow \infty} j_{\ell}^{4} \varepsilon_{j_{\ell}}=0$ and $j_{\ell} \leq \varepsilon_{j_{\ell}}^{1 / 6} / 2$,

(3) $\sup _{\ell \in \mathbb{N}}\left\|\partial \mathcal{E}_{j_{\ell}}\right\|\left(\mathbb{R}^{n+1}\right)<\infty$,

(4) $\liminf \inf _{\ell \rightarrow \infty} \int_{\mathbb{R}^{n+1}} \eta_{j_{\ell}} \frac{\left|\Phi_{\varepsilon_{j_{\ell}}} * \delta\left(\partial \mathcal{E}_{j_{\ell}}\right)\right|^{2}}{\Phi_{\varepsilon_{j_{\ell}}} * \partial \mathcal{E}_{j_{\ell}} \|+\varepsilon_{j_{\ell}}} d x<\infty$,

(5) $\lim _{\ell \rightarrow \infty} \Delta_{j_{\ell}}\left\|\partial \mathcal{E}_{j_{\ell}}\right\|\left(D_{j_{\ell}}\right)=0$. 
Then, there exist a subsequence $\left\{j_{\ell}^{\prime}\right\}_{\ell=1}^{\infty} \subset\left\{j_{\ell}\right\}_{\ell=1}^{\infty}$ and a varifold $V \in \mathbf{V}_{n}\left(\mathbb{R}^{n+1}\right)$ such that $\partial \mathcal{E}_{j_{\ell}^{\prime}} \rightarrow V$ in the sense of varifolds, spt $\|V\| \subset \operatorname{clos} U$, and

$$
\theta^{* n}(\|V\|, x) \geq c_{0}>0 \quad \text { for }\|V\| \text { a.e. } x \in U .
$$

Here, $c_{0}$ is a constant depending only on $n$. Furthermore, $V\left\llcorner\mathbf{G}_{n}(U) \in \mathbf{R V}_{n}(U)\right.$.

Proof The existence of a subsequence $\left\{\partial \mathcal{E}_{j_{\ell}^{\prime}}\right\}_{\ell=1}^{\infty}$ converging in the sense of varifolds to $V \in \mathbf{V}_{n}\left(\mathbb{R}^{n+1}\right)$ follows from the compactness theorem for Radon measures using assumption (3). The limit varifold $V$ satisfies $\operatorname{spt}\|V\| \subset \operatorname{clos} U$ because of assumption (1). Indeed, since $\operatorname{spt}\left\|\partial \mathcal{E}_{j_{\ell}}\right\| \subset \operatorname{clos} U_{j_{\ell}}$ by definition of open partition, if $x \in \mathbb{R}^{n+1} \backslash \operatorname{clos} U$ then (1) implies that there is a radius $r>0$ such that $\left\|\partial \mathcal{E}_{j_{\ell}^{\prime}}\right\|\left(U_{r}(x)\right)=0$ for all sufficiently large $\ell$, which in turn gives $\|V\|\left(U_{r}(x)\right)=0$. Furthermore, the validity of (2), (3), and (4) allows us to apply Proposition 3.20 in order to deduce that $\|\delta V\|\llcorner U$ is a Radon measure. Hence, the rectifiability of the limit varifold in $U$ is a consequence of Allard's rectifiability theorem [1, Theorem 5.5(1)] once we prove (5.4). In turn, the latter can be obtained by repeating verbatim the arguments in [20, Theorem 7.3]. Indeed, the proof in there is local, and for a given $x_{0} \in U$ it can be reproduced by replacing $B_{1}\left(x_{0}\right)$ in [20, Theorem 7.3] by $B_{\rho}\left(x_{0}\right)$ for sufficiently small $\rho>0$ and large $\ell$ so that $B_{\rho}\left(x_{0}\right) \subset D_{j_{\ell}^{\prime}}$ and $\eta_{j_{\ell}^{\prime}}=1$ on $B_{\rho}\left(x_{0}\right)$.

Theorem 5.3 (Integrality Theorem). Under the same assumptions of Theorem 5.2, if the stronger

(5)' $\lim _{\ell \rightarrow \infty} j_{\ell}^{2(n+1)} \Delta_{j_{\ell}}\left\|\partial \mathcal{E}_{j_{\ell}}\right\|\left(D_{j_{\ell}}\right)=0$

holds, then there is a converging subsequence $\left\{\partial \mathcal{E}_{j_{\ell}^{\prime}}\right\}_{\ell=1}^{\infty}$ such that the limit varifold $V$ satisfies $V\left\llcorner\mathbf{G}_{n}(U) \in \mathbf{I V}_{n}(U)\right.$.

Just like Theorem 5.2, the claim is local in nature and the proof is the same as [20, Theorem 8.6].

Proof of Proposition 5.1 First, observe that by (4.29) and Fatou's lemma we have

$$
\liminf _{\ell \rightarrow \infty} \int_{\mathbb{R}^{n+1}} \eta_{j_{\ell}} \frac{\left|\Phi_{\varepsilon_{j_{\ell}}} * \delta\left(\partial \mathcal{E}_{j_{\ell}}(t)\right)\right|^{2}}{\Phi_{\varepsilon_{j_{\ell}}} *\left\|\partial \mathcal{E}_{j_{\ell}}\right\|+\varepsilon_{j_{\ell}}} d x<\infty
$$

for a.e. $t \in \mathbb{R}^{+}$. Furthermore, from (4.3) and the definition of $\partial \mathcal{E}_{j}(t)$ we also have that for every $T<\infty$

$$
\sup _{\ell \in \mathbb{N}} \sup _{t \in[0, T]}\left\|\partial \mathcal{E}_{j_{\ell}}(t)\right\|\left(\mathbb{R}^{n+1}\right)<\infty .
$$

Let $t \in \mathbb{R}^{+}$be such that (5.5) and (4.30) hold. We want to show that the sequence $\left\{\partial \mathcal{E}_{j_{\ell}}(t)\right\}_{\ell=1}^{\infty}$ satisfies the assumptions of Theorem 5.3. Assumption (1) follows from the construction of the discrete flow in Proposition 4.2 and the choice of $\varepsilon_{j \ell}$; (2) follows again from the choice of $\varepsilon_{j_{\ell}}$, more precisely from (3.31); (3) and (4) are (5.6) and (5.5), respectively; (5)' is (4.30). Hence, Theorem 5.3 implies that, along a further subsequence $\left\{j_{\ell}^{\prime}\right\}_{\ell=1}^{\infty} \subset\left\{j_{\ell}\right\}_{\ell=1}^{\infty}, \partial \mathcal{E}_{j_{\ell}^{\prime}}(t)$ converges, as $\ell \rightarrow \infty$, to a varifold $V_{t} \in \mathbf{V}_{n}\left(\mathbb{R}^{n+1}\right)$ with spt $\left\|V_{t}\right\| \subset \operatorname{clos} U$ and such that $V_{t}\left\llcorner\mathbf{G}_{n}(U) \in \mathbf{I V}_{n}(U)\right.$. Since the convergence is in the sense of varifolds, the weights converge as Radon measures, and thus $\lim _{\ell \rightarrow \infty}\left\|\partial \mathcal{E}_{j_{\ell}^{\prime}}(t)\right\|=\left\|V_{t}\right\|:$ (4.27) then readily implies that $\left\|V_{t}\right\|\left\llcorner U=\mu_{t}\right.$ as Radon measures on $U$, thus proving (1). Concerning the statement in (2), let $\left\{j_{\ell}^{\prime}\right\}_{\ell=1}^{\infty}$ be a subsequence along which (5.1) holds. Then, any converging further subsequence must converge to a varifold satisfying the conclusion of Theorem 5.3. A priori, two distinct 
subsequences may converge to different limits. On the other hand, each subsequential limit $V_{t}$ is a rectifiable varifold when restricted to the open set $U$, and furthermore it satisfies $\left\|V_{t}\right\|\left\llcorner U=\mu_{t}\right.$. Since rectifiable varifolds are uniquely determined by their weight, we deduce that the limit in $U$ is independent of the particular subsequence, and thus (5.1) forces the whole sequence $\partial \mathcal{E}_{j_{\ell}^{\prime}}(t)$ to converge to a uniquely determined integral varifold $V_{t}$ in $U$. Finally, (3) follows from Proposition 3.20.

A byproduct of the proof of Proposition 5.1 is the existence of a (uniquely defined) integral varifold $V_{t} \in \mathbf{I} \mathbf{V}_{n}(U)$ with weight $\left\|V_{t}\right\|=\mu_{t}$ for every $t \in \mathbb{R}^{+} \backslash Z$, where $\mathcal{L}^{1}(Z)=0$. Such a varifold $V_{t}$ is the limit on $U$ of any sequence $\partial \mathcal{E}_{j_{\ell}^{\prime}}(t)$ along which (5.1) holds true. We can now extend the definition of $V_{t}$ to $t \in Z$ so to have a one-parameter family $\left\{V_{t}\right\}_{t \in \mathbb{R}^{+}} \subset \mathbf{V}_{n}(U)$ of varifolds satisfying $\left\|V_{t}\right\|=\mu_{t}$ for every $t \in \mathbb{R}^{+}$. Such an extension can be defined in an arbitrary fashion: for instance, if $t \in Z$ then we can set $V_{t}(\varphi):=\int \varphi(x, S) d \mu_{t}(x)$ for every $\varphi \in C_{c}\left(\mathbf{G}_{n}(U)\right)$, where $S$ is any constant plane in $\mathbf{G}(n+1, n)$.

In the next theorem, we show that the family of varifolds $\left\{V_{t}\right\}$ is indeed a Brakke flow in $U$. The boundary condition and the initial condition will be discussed in the following section.

Theorem 5.4 (Brakke's inequality). For every $T>0$ we have

$$
\left\|V_{T}\right\|(U)+\int_{0}^{T} \int_{U}\left|h\left(x, V_{t}\right)\right|^{2} d\left\|V_{t}\right\|(x) d t \leq \mathcal{H}^{n}\left(\Gamma_{0}\right) .
$$

Furthermore, for any $\phi \in C_{c}^{1}\left(U \times \mathbb{R}^{+} ; \mathbb{R}^{+}\right)$and $0 \leq t_{1}<t_{2}<\infty$ we have:

$$
\left.\left\|V_{t}\right\|(\phi(\cdot, t))\right|_{t=t_{1}} ^{t_{2}} \leq \int_{t_{1}}^{t_{2}}\left(\delta\left(V_{t}, \phi(\cdot, t)\right)\left(h\left(\cdot, V_{t}\right)\right)+\left\|V_{t}\right\|\left(\frac{\partial \phi}{\partial t}(\cdot, t)\right)\right) d t .
$$

Proof In order to prove (5.7), we use (4.5) with $\phi=1$ which belongs to $\mathcal{A}_{j} \cap \mathcal{R}_{j}$ for all $j$. Assume $T \in 2_{\mathbb{Q}}$ first. By summing over the index $k$ and for all sufficiently large $j$, we have

$$
\left\|\partial \mathcal{E}_{j}(T)\right\|(U)-\int_{0}^{T} \delta\left(\partial \mathcal{E}_{j}(t)\right)\left(\eta_{j} h_{\varepsilon_{j}}\right) d t \leq \mathcal{H}^{n}\left(\Gamma_{0}\right)+T \varepsilon_{j}^{1 / 8} .
$$

By (3.33) and (5.3) as well as $\left\|V_{T}\right\|(U) \leq \liminf _{\ell \rightarrow \infty}\left\|\partial \mathcal{E}_{j_{\ell}}(T)\right\|(U)$, we obtain (5.7). For $T \notin 2_{\mathbb{Q}}$, use (4.28) to deduce the same inequality.

We now focus on proving the validity of Brakke's inequality (5.8).

Step 1. We will first assume that $\phi$ is independent of $t$, and then extend the proof to the more general case. By an elementary density argument, we can assume that $\phi \in C_{c}^{\infty}\left(U ; \mathbb{R}^{+}\right)$. Moreover, since the support of $\phi$ is compact and (5.8) depends linearly on $\phi$, we can also normalize $\phi$ in such a way that $\phi<1$ everywhere. Then, for all sufficiently large $i \in \mathbb{N}$, also $\hat{\phi}:=\phi+i^{-1}<1$ everywhere. Arguing as in the proof of Proposition 4.13, we can choose $m \in \mathbb{N}$ so that $m \geq J(n)$ (see Lemma 3.16) and furthermore

(i) $\hat{\phi} \in \mathcal{A}_{j} \cap \mathcal{R}_{j}$,

(ii) $\eta_{j} \hat{\phi} \in \mathcal{A}_{j}$

for all $j \geq m$. Next, fix $0 \leq t_{1}<t_{2}<\infty$, and let $\ell$ be such that $j_{\ell} \geq m$ and $j_{\ell} \geq t_{2}$, so that $\partial \mathcal{E}_{j_{\ell}}(t)$ is certainly well defined for $t \in\left[t_{1}, t_{2}\right]$. By the condition (i) above, we can apply (4.5) with $\hat{\phi}$ and deduce

$$
\left\|\partial \mathcal{E}_{j_{\ell}}(t)\right\|(\hat{\phi})-\left\|\partial \mathcal{E}_{j_{\ell}}\left(t-\Delta t_{j_{\ell}}\right)\right\|(\hat{\phi}) \leq \Delta t_{j_{\ell}}\left(\delta\left(\partial \mathcal{E}_{j_{\ell}}(t), \hat{\phi}\right)\left(\eta_{j_{\ell}} h_{\varepsilon_{j_{\ell}}}\left(\cdot, \partial \mathcal{E}_{j_{\ell}}(t)\right)\right)+\varepsilon_{j_{\ell}}^{1 / 8}\right)
$$


for every $t=\Delta t_{j_{\ell}}, 2 \Delta t_{j_{\ell}}, \ldots, j_{\ell} 2^{p_{j_{\ell}}} \Delta t_{j_{\ell}}$. Since $\Delta t_{j_{\ell}} \rightarrow 0$ as $\ell \rightarrow \infty$, we can assume without loss of generality that $\Delta t_{j \ell}<t_{2}-t_{1}$, so that there exist $k_{1}, k_{2} \in \mathbb{N}$ with $k_{1}<k_{2}$ such that $t_{1} \in\left(\left(k_{1}-2\right) \Delta t_{j_{\ell}},\left(k_{1}-1\right) \Delta t_{j_{\ell}}\right]$ and $t_{2} \in\left(\left(k_{2}-1\right) \Delta t_{j_{\ell}}, k_{2} \Delta t_{j_{\ell}}\right]$. If we sum (5.9) on $t=k \Delta t_{j \ell}$ for $k \in\left[k_{1}, k_{2}\right] \cap \mathbb{N}$ we get

$$
\begin{aligned}
& \left.\left\|\partial \mathcal{E}_{j_{\ell}}(t)\right\|(\hat{\phi})\right|_{t=\left(k_{1}-1\right) \Delta t_{j_{\ell}}} ^{k_{2} \Delta t_{j_{\ell}}} \\
& \leq \sum_{k=k_{1}}^{k_{2}} \Delta t_{j_{\ell}}\left(\delta\left(\partial \mathcal{E}_{j_{\ell}}\left(k \Delta t_{j_{\ell}}\right), \hat{\phi}\right)\left(\eta_{j_{\ell}} h_{\varepsilon_{j_{\ell}}}\left(\cdot, \partial \mathcal{E}_{j_{\ell}}\left(k \Delta t_{j_{\ell}}\right)\right)\right)+\varepsilon_{j_{\ell}}^{1 / 8}\right) .
\end{aligned}
$$

Since $\hat{\phi}=\phi+i^{-1}$, we can estimate the left-hand side of (5.10) from below as

$$
\left.\left\|\partial \mathcal{E}_{j_{\ell}}(t)\right\|(\hat{\phi})\right|_{t=\left(k_{1}-1\right) \Delta t_{j_{\ell}}} ^{k_{2} \Delta t_{j_{\ell}}} \geq\left\|\partial \mathcal{E}_{j_{\ell}}\left(t_{2}\right)\right\|(\phi)-\left\|\partial \mathcal{E}_{j_{\ell}}\left(t_{1}\right)\right\|(\phi)-i^{-1}\left\|\partial \mathcal{E}_{j_{\ell}}\left(t_{1}\right)\right\|\left(\mathbb{R}^{n+1}\right),
$$

so that when we let $\ell \rightarrow \infty$ we conclude

$$
\left.\limsup _{\ell \rightarrow \infty}\left\|\partial \mathcal{E}_{j_{\ell}}(t)\right\|(\hat{\phi})\right|_{t=\left(k_{1}-1\right) \Delta t_{j_{\ell}}} ^{k_{2} \Delta t_{j_{\ell}}} \geq\left.\left\|V_{t}\right\|(\phi)\right|_{t=t_{1}} ^{t_{2}}-i^{-1}\left\|\partial \mathcal{E}_{0}\right\|\left(\mathbb{R}^{n+1}\right),
$$

where we have used (4.27) together with Proposition 5.1(1).

Next, we estimate the right-hand side of (5.10) from above. Setting $\partial \mathcal{E}_{j_{\ell}}=\partial \mathcal{E}_{j_{\ell}}(t)$ and $h_{\varepsilon_{j_{\ell}}}=h_{\varepsilon_{j_{\ell}}}\left(\cdot, \partial \mathcal{E}_{j_{\ell}}\right)$, we proceed as in (4.35) writing

$$
\delta\left(\partial \mathcal{E}_{j_{\ell}}, \hat{\phi}\right)\left(\eta_{j_{\ell}} h_{\varepsilon_{j_{\ell}}}\right)=\delta\left(\partial \mathcal{E}_{j_{\ell}}\right)\left(\eta_{j_{\ell}} \hat{\phi} h_{\varepsilon_{j_{\ell}}}\right)+\int_{\mathbf{G}_{n}\left(\mathbb{R}^{n+1}\right)} \eta_{j_{\ell}} S^{\perp}(\nabla \phi) \cdot h_{\varepsilon_{j_{\ell}}} d\left(\partial \mathcal{E}_{j_{\ell}}\right),
$$

where we have used that $\nabla \hat{\phi}=\nabla \phi$. Since $\eta_{j_{\ell}} \hat{\phi} \in \mathcal{A}_{j_{\ell}}$, we can apply (3.33) in order to obtain that

$$
\left|\delta\left(\partial \mathcal{E}_{j_{\ell}}\right)\left(\eta_{j_{\ell}} \hat{\phi} h_{\varepsilon_{j_{\ell}}}\right)+b_{j_{\ell}}\right| \leq \varepsilon_{j_{\ell}}^{1 / 4}\left(b_{j_{\ell}}+1\right),
$$

where we have set for simplicity

$$
b_{j_{\ell}}:=\int_{\mathbb{R}^{n+1}} \eta_{j_{\ell}} \hat{\phi} \frac{\left|\Phi_{\varepsilon_{j_{\ell}}} * \delta\left(\partial \mathcal{E}_{j_{\ell}}\right)\right|^{2}}{\Phi_{\varepsilon_{j_{\ell}}} *\left\|\mathcal{E}_{j_{\ell}}\right\|+\varepsilon_{j_{\ell}}} d x .
$$

Concerning the second summand in (5.13), we use the Cauchy-Schwarz inequality to estimate

$$
\begin{aligned}
\left|\int_{\mathbf{G}_{n}\left(\mathbb{R}^{n+1}\right)} \eta_{j_{\ell}} S^{\perp}(\nabla \phi) \cdot h_{\varepsilon_{j_{\ell}}} d\left(\partial \mathcal{E}_{j_{\ell}}\right)\right| & \leq\left(\int_{\mathbb{R}^{n+1}} \eta_{j_{\ell}} \frac{|\nabla \phi|^{2}}{\hat{\phi}}\right)^{1 / 2}\left(\int_{\mathbb{R}^{n+1}} \eta_{j_{\ell}} \hat{\phi}\left|h_{\varepsilon_{j_{\ell}}}\right|^{2}\right)^{1 / 2} \\
& \leq c\left\|\partial \mathcal{E}_{j_{\ell}}\right\|\left(\mathbb{R}^{n+1}\right)^{1 / 2}\left(\left(1+\varepsilon_{j_{\ell}}^{1 / 4}\right) b_{j_{\ell}}+\varepsilon_{j_{\ell}}^{1 / 4}\right)^{1 / 2}
\end{aligned}
$$

where $c$ depends only on $\|\phi\|_{C^{2}}$, and where we have used (3.34). Using (5.14), (5.16) and (4.3), we can then conclude that

$$
\sup _{t \in\left[t_{1}, t_{2}\right]} \delta\left(\partial \mathcal{E}_{j_{\ell}}(t), \hat{\phi}\right)\left(\eta_{j_{\ell}} h_{\varepsilon_{j_{\ell}}}\left(\cdot, \partial \mathcal{E}_{j_{\ell}}(t)\right)\right) \leq c,
$$


where $c$ depends only on $\|\phi\|_{C^{2}}$ and $\left\|\partial \mathcal{E}_{0}\right\|\left(\mathbb{R}^{n+1}\right)$. Using (5.17) together with the definition of $\partial \mathcal{E}_{j_{\ell}}(t)$ and Fatou's lemma, one can readily show that, when we take the lim sup as $\ell \rightarrow \infty$, the right-hand side of (5.10) can be bounded by

$$
\begin{aligned}
& \limsup _{\ell \rightarrow \infty} \sum_{k=k_{1}}^{k_{2}} \Delta t_{j_{\ell}}\left(\delta\left(\partial \mathcal{E}_{j_{\ell}}\left(k \Delta t_{j_{\ell}}\right), \hat{\phi}\right)\left(\eta_{j_{\ell}} h_{\varepsilon_{j_{\ell}}}\left(\cdot, \partial \mathcal{E}_{j_{\ell}}\left(k \Delta t_{j_{\ell}}\right)\right)\right)+\varepsilon_{j_{\ell}}^{1 / 8}\right) \\
& \quad=\limsup _{\ell \rightarrow \infty} \int_{t_{1}}^{t_{2}} \delta\left(\partial \mathcal{E}_{j_{\ell}}(t), \hat{\phi}\right)\left(\eta_{j_{\ell}} h_{\varepsilon_{j_{\ell}}}\left(\cdot, \partial \mathcal{E}_{j_{\ell}}(t)\right)\right) d t \\
& \quad \leq \int_{t_{1}}^{t_{2}} \limsup _{\ell \rightarrow \infty} \delta\left(\partial \mathcal{E}_{j_{\ell}}(t), \hat{\phi}\right)\left(\eta_{j_{\ell}} h_{\varepsilon_{j_{\ell}}}\left(\cdot, \partial \mathcal{E}_{j_{\ell}}(t)\right)\right) d t
\end{aligned}
$$

Now, fix $t \in\left[t_{1}, t_{2}\right]$ such that $\liminf _{\ell \rightarrow \infty} b_{j_{\ell}}<\infty$ (which holds for a.e. $t$ ), and let $\left\{j_{\ell}^{\prime}\right\} \subset\left\{j_{\ell}\right\}$ be a subsequence which realizes the lim sup, namely with

$$
\lim _{\ell \rightarrow \infty} \delta\left(\partial \mathcal{E}_{j_{\ell}^{\prime}}(t), \hat{\phi}\right)\left(\eta_{j_{\ell}^{\prime}} h_{\varepsilon_{j_{\ell}^{\prime}}}\left(\cdot, \partial \mathcal{E}_{j_{\ell}^{\prime}}(t)\right)\right)=\limsup _{\ell \rightarrow \infty} \delta\left(\partial \mathcal{E}_{j_{\ell}}(t), \hat{\phi}\right)\left(\eta_{j_{\ell}} h_{\varepsilon_{j_{\ell}}}\left(\cdot, \partial \mathcal{E}_{j_{\ell}}(t)\right)\right)
$$

By the identity in (5.13), we also have that along the same subsequence

$$
\begin{aligned}
& \lim _{\ell \rightarrow \infty}\left(-\delta\left(\partial \mathcal{E}_{j_{\ell}^{\prime}}\right)\left(\eta_{j_{\ell}^{\prime}} \hat{\phi} h_{\varepsilon_{j_{\ell}^{\prime}}}\right)-\int_{\mathbf{G}_{n}\left(\mathbb{R}^{n+1}\right)} \eta_{j_{\ell}^{\prime}} S^{\perp}(\nabla \phi) \cdot h_{\varepsilon_{j_{\ell}^{\prime}}} d\left(\partial \mathcal{E}_{j_{\ell}^{\prime}}\right)\right) \\
& \quad=\liminf _{\ell \rightarrow \infty}\left(-\delta\left(\partial \mathcal{E}_{j_{\ell}}\right)\left(\eta_{j_{\ell}} \hat{\phi} h_{\varepsilon_{j_{\ell}}}\right)-\int_{\mathbf{G}_{n}\left(\mathbb{R}^{n+1}\right)} \eta_{j_{\ell}} S^{\perp}(\nabla \phi) \cdot h_{\varepsilon_{j_{\ell}}} d\left(\partial \mathcal{E}_{j_{\ell}}\right)\right),
\end{aligned}
$$

where once again $\partial \mathcal{E}_{j_{\ell}}=\partial \mathcal{E}_{j_{\ell}}(t)$ and $h_{\varepsilon_{j_{\ell}}}=h_{\varepsilon_{j_{\ell}}}\left(\cdot, \partial \mathcal{E}_{j_{\ell}}\right)$. Using (5.14) and (5.16), we see that the right-hand side of (5.20) can be bounded from above by $\lim _{\inf _{\ell \rightarrow \infty}} 2 b_{j_{\ell}}+c$, whereas the left-hand side can be bounded from below by $\lim \sup _{\ell \rightarrow \infty} \frac{1}{2} b_{j_{\ell}^{\prime}}-c$, where $c$ depends on $\|\phi\|_{C^{2}}$ and $\left\|\partial \mathcal{E}_{0}\right\|\left(\mathbb{R}^{n+1}\right)$. As a consequence, along any subsequence $\left\{j_{\ell}^{\prime}\right\}$ satisfying (5.19) one has that

$$
\begin{aligned}
& \limsup _{\ell \rightarrow \infty} \int_{\mathbb{R}^{n+1}} \eta_{j_{\ell}^{\prime}} \hat{\phi} \frac{\left|\Phi_{\varepsilon_{j_{\ell}^{\prime}}} * \delta\left(\partial \mathcal{E}_{j_{\ell}^{\prime}}\right)\right|^{2}}{\Phi_{\varepsilon_{j_{\ell}}} *\left\|\partial \mathcal{E}_{j_{\ell}^{\prime}}\right\|+\varepsilon_{j_{\ell}^{\prime}}} d x \\
& \leq 4 \liminf _{\ell \rightarrow \infty} \int_{\mathbb{R}^{n+1}} \eta_{j_{\ell}} \hat{\phi} \frac{\left|\Phi_{\varepsilon_{j_{\ell}}} * \delta\left(\partial \mathcal{E}_{j_{\ell}}\right)\right|^{2}}{\Phi_{\varepsilon_{j_{\ell}}} *\left\|\partial \mathcal{E}_{j_{\ell}}\right\|+\varepsilon_{j_{\ell}}} d x+c<\infty,
\end{aligned}
$$

where $\partial \mathcal{E}_{j_{\ell}^{\prime}}=\partial \mathcal{E}_{j_{\ell}^{\prime}}(t)$. Let us denote the right-hand side of (5.21) as $B(t)$. Since $\hat{\phi} \geq i^{-1}$, and thanks to (5.21), if $B(t)<\infty$ then the assumption (5.1) of Proposition 5.1 is satisfied along $j_{\ell}^{\prime}$ : hence, the whole sequence $\left\{\partial \mathcal{E}_{j_{\ell}^{\prime}}(t)\right\}_{\ell=1}^{\infty}$ converges to $V_{t} \in \mathbf{I V}_{n}(U)$ as varifolds in $U$. Furthermore, using one more time that $\hat{\phi} \geq i^{-1}$ we deduce that

$$
\limsup _{\ell \rightarrow \infty} \int_{\mathbb{R}^{n+1}} \eta_{j_{\ell}^{\prime}} \frac{\left|\Phi_{\varepsilon_{j_{\ell}^{\prime}}} * \delta\left(\partial \mathcal{E}_{j_{\ell}^{\prime}}\right)\right|^{2}}{\Phi_{\varepsilon_{j_{\ell}^{\prime}}} *\left\|\mathcal{E}_{j_{\ell}^{\prime}}\right\|+\varepsilon_{j_{\ell}^{\prime}}} d x \leq i B(t) .
$$


Using (5.19), (5.13), (5.14), $\hat{\phi}>\phi$, and Proposition 5.1(3) with $\phi$ (recalling $\phi \in$ $\left.C_{c}^{\infty}\left(U ; \mathbb{R}^{+}\right)\right)$, we have

$$
\begin{aligned}
& \limsup _{\ell \rightarrow \infty} \delta\left(\partial \mathcal{E}_{j_{\ell}}(t), \hat{\phi}\right)\left(\eta_{j_{\ell}} h_{\varepsilon_{j_{\ell}}}\left(\cdot, \partial \mathcal{E}_{j_{\ell}}(t)\right)\right)=\lim _{\ell \rightarrow \infty} \delta\left(\partial \mathcal{E}_{j_{\ell}^{\prime}}(t), \hat{\phi}\right)\left(\eta_{j_{\ell}^{\prime}} h_{\varepsilon_{j_{\ell}^{\prime}}}\left(\cdot, \partial \mathcal{E}_{j_{\ell}^{\prime}}(t)\right)\right) \\
& \leq-\int_{U}\left|h\left(\cdot, V_{t}\right)\right|^{2} \phi d\left\|V_{t}\right\| \\
& \quad+\limsup _{\ell \rightarrow \infty} \int_{\mathbf{G}_{n}(U)} S^{\perp}(\nabla \phi) \cdot h_{\varepsilon_{j_{\ell}^{\prime}}}\left(\cdot, \partial \mathcal{E}_{j_{\ell}^{\prime}}(t)\right) d\left(\partial \mathcal{E}_{j_{\ell}^{\prime}}(t)\right)
\end{aligned}
$$

where we have also used that, as $\ell \rightarrow \infty, \eta_{j_{\ell}^{\prime}}=1$ on $\{\nabla \phi \neq 0\} \subset \subset U$.

Now, recall that $V_{t} \in \mathbf{I V}_{n}(U)$. Therefore, there is an $\mathcal{H}^{n}$-rectifiable set $M_{t} \subset U$ such that

$$
\int_{\mathbf{G}_{n}(U)} S^{\perp}(\nabla \phi(x)) d V_{t}(x, S)=\int_{U} T_{x} M_{t}^{\perp}(\nabla \phi(x)) d\left\|V_{t}\right\|(x) .
$$

Furthermore, since the map $x \mapsto T_{x} M_{t}^{\perp}(\nabla \phi(x))$ is in $L^{2}\left(\left\|V_{t}\right\|\right)$, for any $\varepsilon>0$ there are a vector field $g \in C_{c}^{\infty}\left(U ; \mathbb{R}^{n+1}\right)$ and a positive integer $m^{\prime}$ such that $g \in \mathcal{B}_{m^{\prime}}$ and

$$
\int_{U}\left|T_{x} M_{t}^{\perp}(\nabla \phi(x))-g(x)\right|^{2} d\left\|V_{t}\right\|(x) \leq \varepsilon^{2} .
$$

In order to estimate the lim sup in the right-hand side of (5.23), we can now compute, for $\partial \mathcal{E}_{j_{\ell}^{\prime}}=\partial \mathcal{E}_{j_{\ell}^{\prime}}(t)$

$$
\begin{aligned}
& \int_{\mathbf{G}_{n}(U)} S^{\perp}(\nabla \phi) \cdot h_{\varepsilon_{j_{\ell}^{\prime}}}\left(\cdot, \partial \mathcal{E}_{j_{\ell}^{\prime}}\right) d\left(\partial \mathcal{E}_{j_{\ell}^{\prime}}\right) \\
& =\int_{\mathbf{G}_{n}(U)}\left(S^{\perp}(\nabla \phi)-g\right) \cdot h_{\varepsilon_{j_{\ell}^{\prime}}} d\left(\partial \mathcal{E}_{j_{\ell}^{\prime}}\right) \\
& \quad+\left(\int_{U} g \cdot h_{\varepsilon_{j_{\ell}^{\prime}}} d\left\|\partial \mathcal{E}_{j_{\ell}^{\prime}}\right\|+\delta\left(\partial \mathcal{E}_{j_{\ell}^{\prime}}\right)(g)\right)-\delta\left(\partial \mathcal{E}_{j_{\ell}^{\prime}}\right)(g)+\delta V_{t}(g) \\
& \quad+\int_{U} h\left(\cdot, V_{t}\right) \cdot\left(g-T \cdot M_{t}^{\perp}(\nabla \phi)\right) d\left\|V_{t}\right\| \\
& \quad+\int_{\mathbf{G}_{n}(U)} h\left(\cdot, V_{t}\right) \cdot S^{\perp}(\nabla \phi) d V_{t}(\cdot, S) .
\end{aligned}
$$

We proceed estimating each term of (5.26). Using that $\eta_{j_{\ell}^{\prime}}=1$ on $\{\nabla \phi \neq 0\}$ for all $\ell$ sufficiently large, the Cauchy-Schwarz inequality gives that

$$
\begin{aligned}
& \left|\int_{\mathbf{G}_{n}(U)}\left(S^{\perp}(\nabla \phi)-g\right) \cdot h_{\varepsilon_{j_{\ell}^{\prime}}} d\left(\partial \mathcal{E}_{j_{\ell}^{\prime}}\right)\right| \\
& \quad \leq\left(\int_{\mathbf{G}_{n}(U)}\left|S^{\perp}(\nabla \phi)-g\right|^{2} d\left(\partial \mathcal{E}_{j_{\ell}^{\prime}}\right)\right)^{\frac{1}{2}}\left(\int_{\mathbb{R}^{n+1}} \eta_{j_{\ell}^{\prime}}\left|h_{\varepsilon_{j_{\ell}^{\prime}}}\right|^{2} d\left\|\partial \mathcal{E}_{j_{\ell}^{\prime}}\right\|\right)^{\frac{1}{2}}
\end{aligned}
$$

for all $\ell$ sufficiently large. Since $(x, S) \mapsto\left|S^{\perp}(\nabla \phi(x))-g(x)\right|^{2} \in C_{c}\left(\mathbf{G}_{n}(U)\right)$, we have that

$$
\begin{aligned}
\lim _{\ell \rightarrow \infty} \int_{\mathbf{G}_{n}(U)}\left|S^{\perp}(\nabla \phi)-g\right|^{2} d\left(\partial \mathcal{E}_{j_{\ell}^{\prime}}\right) & =\int_{\mathbf{G}_{n}(U)}\left|S^{\perp}(\nabla \phi)-g\right|^{2} d V_{t} \\
& =\int_{U}\left|T_{x} M_{t}^{\perp}(\nabla \phi(x))-g(x)\right|^{2} d\left\|V_{t}\right\|(x) \stackrel{(5.25)}{\leq} \varepsilon^{2} .
\end{aligned}
$$


Using (3.34), (5.22), (5.27) and (5.28), we then conclude that

$$
\limsup _{\ell \rightarrow \infty}\left|\int_{\mathbf{G}_{n}(U)}\left(S^{\perp}(\nabla \phi)-g\right) \cdot h_{\varepsilon_{j_{\ell}^{\prime}}} d\left(\partial \mathcal{E}_{j_{\ell}^{\prime}}\right)\right| \leq(i B(t))^{\frac{1}{2}} \varepsilon .
$$

Analogously, since $\eta_{j_{\ell}^{\prime}}=1$ on $\{g \neq 0\}$ for all $\ell$ sufficiently large, we have that

$$
\begin{aligned}
& \lim _{\ell \rightarrow \infty}\left|\int_{U} g \cdot h_{\varepsilon_{j_{\ell}^{\prime}}} d\left\|\partial \mathcal{E}_{j_{\ell}^{\prime}}\right\|+\delta\left(\partial \mathcal{E}_{j_{\ell}^{\prime}}\right)(g)\right| \\
& =\lim _{\ell \rightarrow \infty}\left|\int_{\mathbb{R}^{n+1}} \eta_{j_{\ell}^{\prime}} g \cdot h_{\varepsilon_{j_{\ell}^{\prime}}} d\left\|\partial \mathcal{E}_{j_{\ell}^{\prime}}\right\|+\delta\left(\partial \mathcal{E}_{j_{\ell}^{\prime}}\right)\left(\eta_{j_{\ell}^{\prime}} g\right)\right|=0
\end{aligned}
$$

by (3.35) and (5.22).

Next, by varifold convergence of $\partial \mathcal{E}_{j_{\ell}^{\prime}}$ to $V_{t}$ on $U$, given that $g$ has compact support in $U$, we also have

$$
\lim _{\ell \rightarrow \infty}\left|\delta\left(\partial \mathcal{E}_{j_{\ell}^{\prime}}\right)(g)-\delta V_{t}(g)\right|=0 .
$$

Finally, letting $\psi$ be any function in $C_{c}\left(U ; \mathbb{R}^{+}\right)$such that $\psi=1$ on $\{g \neq 0\} \cup\{\nabla \phi \neq 0\}$ and $0 \leq \psi \leq 1$, the Cauchy-Schwarz inequality allows us to estimate

$$
\begin{aligned}
& \left|\int_{U} h\left(x, V_{t}\right) \cdot\left(g(x)-T_{x} M_{t}^{\perp}(\nabla \phi(x))\right) d\left\|V_{t}\right\|\right| \\
& \quad \leq\left(\int_{U}\left|h\left(x, V_{t}\right)\right|^{2} \psi(x) d\left\|V_{t}\right\|(x)\right)^{\frac{1}{2}}\left(\int_{U}\left|g(x)-T_{x} M_{t}^{\perp}(\nabla \phi(x))\right|^{2} d\left\|V_{t}\right\|(x)\right)^{\frac{1}{2}} \\
& \quad \leq(i B(t))^{\frac{1}{2}} \varepsilon,
\end{aligned}
$$

where in the last inequality we have used (5.3) with $\psi$ in place of $\phi,(5.22)$ and (5.25).

From (5.26), combining (5.29)-(5.32) we conclude that

$$
\limsup _{\ell \rightarrow \infty} \int_{\mathbf{G}_{n}(U)} S^{\perp}(\nabla \phi) \cdot h_{\varepsilon_{j_{\ell}^{\prime}}}\left(\cdot, \partial \mathcal{E}_{j_{\ell}^{\prime}}\right) d\left(\partial \mathcal{E}_{j_{\ell}^{\prime}}\right) \leq \int_{U} h\left(\cdot, V_{t}\right) \cdot \nabla \phi d\left\|V_{t}\right\|+2(i B(t))^{\frac{1}{2}} \varepsilon,
$$

where we have also used (2.6).

We can now combine (5.10), (5.12), (5.18), (5.23), and (5.33) to deduce that

$$
\begin{aligned}
\left.\left\|V_{t}\right\|(\phi)\right|_{t=t_{1}} ^{t_{2}} \leq & -\int_{t_{1}}^{t_{2}} \int_{U}\left(\left|h\left(\cdot, V_{t}\right)\right|^{2} \phi-h\left(\cdot, V_{t}\right) \cdot \nabla \phi\right) d\left\|V_{t}\right\| d t \\
& +i^{-1}\left\|\partial \mathcal{E}_{0}\right\|\left(\mathbb{R}^{n+1}\right)+2 i^{\frac{1}{2}} \varepsilon \int_{t_{1}}^{t_{2}} B(t)^{\frac{1}{2}} d t .
\end{aligned}
$$

We use the Cauchy-Schwarz inequality one more time, and combine it with the definition of $B(t)$ as the right-hand side of (5.21) and with Fatou's lemma to obtain the bound

$$
\int_{t_{1}}^{t_{2}} B(t)^{\frac{1}{2}} d t \leq\left(t_{2}-t_{1}\right)+c\left(t_{2}-t_{1}\right)+4 \liminf _{\ell \rightarrow \infty} \int_{t_{1}}^{t_{2}} \int_{\mathbb{R}^{n+1}} \eta_{j_{\ell}} \hat{\phi} \frac{\left|\Phi_{\varepsilon_{j_{\ell}}} * \delta\left(\partial \mathcal{E}_{j_{\ell}}\right)\right|^{2}}{\Phi_{\varepsilon_{j_{\ell}}} *\left\|\partial \mathcal{E}_{j_{\ell}}\right\|+\varepsilon_{j_{\ell}}},
$$


which is finite (depending on $t_{2}$ ) by (4.29) (recall that $\hat{\phi} \leq 1$ everywhere). Brakke's inequality (5.8) for a test function $\phi$ which does not depend on $t$ is then deduced from (5.34) after letting $\varepsilon \downarrow 0$ and then $i \uparrow \infty$.

Step 2. We consider now the general case of a time dependent test function $\phi \in C_{c}^{1}(U \times$ $\mathbb{R}^{+} ; \mathbb{R}^{+}$). We can once again assume that $\phi$ is smooth, and then conclude by a density argument. The proof follows the same strategy of Step 1. We define $\hat{\phi}$ analogously, and then we apply (4.5) with $\phi=\hat{\phi}(\cdot, t)$. In place of (5.9), we then obtain a formula with one extra term, namely

$$
\begin{aligned}
& \left.\left\|\partial \mathcal{E}_{j_{\ell}}(s)\right\|(\hat{\phi}(\cdot, s))\right|_{s=t-\Delta t_{j_{\ell}}} ^{t} \leq \Delta t_{j_{\ell}}\left(\delta\left(\partial \mathcal{E}_{j_{\ell}}(t), \hat{\phi}(\cdot, t)\right)\left(\eta_{j_{\ell}} h_{\varepsilon_{j_{\ell}}}\left(\cdot, \partial \mathcal{E}_{j_{\ell}}(t)\right)\right)+\varepsilon_{j_{\ell}}^{1 / 8}\right) \\
& \quad+\left\|\partial \mathcal{E}_{j_{\ell}}\left(t-\Delta t_{j_{\ell}}\right)\right\|\left(\phi(\cdot, t)-\phi\left(\cdot, t-\Delta t_{j_{\ell}}\right)\right) .
\end{aligned}
$$

Similarly, the inequality in (5.10) needs to be replaced with an analogous one containing, in the right-hand side, also the term

$$
\sum_{k=k_{1}}^{k_{2}}\left\|\partial \mathcal{E}_{j_{\ell}}\left((k-1) \Delta t_{j_{\ell}}\right)\right\|\left(\phi\left(\cdot, k \Delta t_{j_{\ell}}\right)-\phi\left(\cdot,(k-1) \Delta t_{j_{\ell}}\right)\right) .
$$

Using the regularity of $\phi$ and the estimates in (4.3) and (4.4), we may deduce that

$$
\begin{aligned}
\lim _{\ell \rightarrow \infty}(5.37) & =\lim _{\ell \rightarrow \infty} \sum_{k=k_{1}}^{k_{2}}\left\|\partial \mathcal{E}_{j_{\ell}}\left(k \Delta t_{j_{\ell}}\right)\right\|\left(\frac{\partial \phi}{\partial t}\left(\cdot, k \Delta t_{j_{\ell}}\right)\right) \Delta t_{j \ell} \\
& =\lim _{\ell \rightarrow \infty} \int_{t_{1}}^{t_{2}}\left\|\partial \mathcal{E}_{j_{\ell}}(t)\right\|\left(\frac{\partial \phi}{\partial t}(\cdot, t)\right) d t \\
& =\int_{t_{1}}^{t_{2}}\left\|V_{t}\right\|\left(\frac{\partial \phi}{\partial t}(\cdot, t)\right) d t,
\end{aligned}
$$

where the last identity is a consequence of (4.27), Proposition 5.1(1), and Lebesgue's dominated convergence theorem. The remaining part of the argument stays the same, modulo the following variation. The identity in (5.18) remains true if $\hat{\phi}$ is replaced by the piecewise constant function $\hat{\phi}_{j \ell}$ defined by

$$
\hat{\phi}_{j_{\ell}}(x, t):=\hat{\phi}\left(x, k \Delta t_{j_{\ell}}\right) \quad \text { if } t \in\left((k-1) \Delta t_{j_{\ell}}, k \Delta t_{j_{\ell}}\right] .
$$

The error one makes in order to put $\hat{\phi}$ back into (5.18) in place of $\hat{\phi}_{j_{\ell}}$ is then given by the product of $\Delta t_{j_{\ell}}$ times some negative powers of $\varepsilon_{j_{\ell}}$; nonetheless, this error converges to 0 uniformly as $\ell \uparrow \infty$ by the choice of $\Delta t_{j \ell}$, see (4.8). This allows us to conclude the proof of (5.8) precisely as in the case of a time-independent $\phi$ whenever $\phi \in C_{c}^{\infty}\left(U \times \mathbb{R}^{+} ; \mathbb{R}^{+}\right)$, and in turn, by approximation, also when $\phi \in C_{c}^{1}\left(U \times \mathbb{R}^{+} ; \mathbb{R}^{+}\right)$.

\section{Boundary behavior and proof of main results}

\subsection{Vanishing of measure outside the convex hull of initial data}

First, we prove that the limit measures $\left\|V_{t}\right\|$ vanish uniformly in time near $\partial U \backslash \partial \Gamma_{0}$. This is a preliminary result, and using the Brakke's inquality, we eventually prove that they actually vanish outside the convex hull of $\Gamma_{0} \cup \partial \Gamma_{0}$ in Proposition 6.4. 
Proposition 6.1 For $\hat{x} \in \partial U \backslash \partial \Gamma_{0}$, suppose that an affine hyperplane $A \subset \mathbb{R}^{n+1}$ with $\hat{x} \notin A$ has the following property. Let $A^{+}$and $A^{-}$be defined as the open half-spaces separated by $A$, i.e., $\mathbb{R}^{n+1}$ is a disjoint union of $A^{+}, A$ and $A^{-}$, with $\hat{x} \in A^{+}$. Define $d_{A}(x):=\operatorname{dist}\left(x, A^{-}\right)$, and suppose that

(1) $\Gamma_{0} \cup \partial \Gamma_{0} \subset A^{-}$,

(2) $d_{A}$ is $v_{U}$-non decreasing in $A^{+}$.

Then for any compact set $C \subset A^{+}$, we have

$$
\lim _{j \rightarrow \infty} \sup _{t \in\left[0, j^{1 / 2}\right]}\left\|\partial \mathcal{E}_{j}(t)\right\|(C)=0 .
$$

Remark 6.2 Due to the definition of $\partial \Gamma_{0}$ and the strict convexity of $U$, note that there exists such an affine hyperplane $A$ for any given $\hat{x} \in \partial U \backslash \partial \Gamma_{0}$. For example, we may choose a hyperplane $A$ which is parallel to the tangent space of $\partial U$ at $\hat{x}$ and which passes through $\hat{x}-v_{U}(\hat{x}) c$. By the strict convexity of $U$ and the $C^{1}$ regularity of $v_{U}$, for all sufficiently small $c>0$, one can show that such $A$ satisfies the above (1) and (2).

Remark 6.3 In the following proof, we adapted a computation from [17, p.60]. There, the object is the Brakke flow, but the basic idea here is that a similar computation can be carried out for the approximate MCF with suitable error estimates.

Proof We may assume after a suitable change of coordinates that $A=\left\{x_{n+1}=0\right\}$ and $A^{+}=\left\{x_{n+1}>0\right\}$. With this, we have clos $\Gamma_{0} \subset\left\{x_{n+1}<0\right\}$ and $d_{A}(x)=\max \left\{x_{n+1}, 0\right\}$ is $v_{U}$-non decreasing in $\left\{x_{n+1}>0\right\}$. Let $s>0$ be arbitrary, and define

$$
\phi(x):=s+\left(d_{A}(x)\right)^{\beta}
$$

for some $\beta \geq 3$ to be fixed later. Then $\phi \in C^{2}\left(\mathbb{R}^{n+1} ; \mathbb{R}^{+}\right)$, and letting $\left\{e_{1}, \ldots, e_{n+1}\right\}$ denote the standard basis of $\mathbb{R}^{n+1}$, we have

$$
\nabla \phi=\beta d_{A}^{\beta-1} e_{n+1}, \quad \nabla^{2} \phi=\beta(\beta-1) d_{A}^{\beta-2} e_{n+1} \otimes e_{n+1} .
$$

With $s>0$ fixed, we choose sufficiently large $j$ so that $\phi \in \mathcal{A}_{j}$. Actually, the function $\phi$ as defined in (6.2) is unbounded. Nonetheless, since we know that spt $\left\|\partial \mathcal{E}_{j}(t)\right\| \subset(U)_{1 /\left(4 j^{1 / 4}\right)}$, we may modify $\phi$ suitably away from $U$ by multiplying it by a small number and truncating it, so that $\phi \leq 1$. We assume that we have done this modification if necessary. We also choose $j$ so large that $\eta_{j}=1$ on $\left\{x_{n+1} \geq 0\right\}$. This is possible due to Lemma 3.16(1). Additionally, since $d_{A}$ is $v_{U}$-non decreasing in $A^{+}$, and since $\phi$ is constant in $\mathbb{R}^{n+1} \backslash A^{+}$, we have $\phi \in \mathcal{R}_{j}$. Thus, by (4.5), we have for $\partial \mathcal{E}_{j, k}=: V$ and $\partial \mathcal{E}_{j, k-1}=: \hat{V}$ with $k \in\left\{1, \ldots, j 2^{p_{j}}\right\}$

$$
\frac{\|V\|(\phi)-\|\hat{V}\|(\phi)}{\Delta t_{j}} \leq \varepsilon_{j}^{1 / 8}+\delta(V, \phi)\left(\eta_{j} h_{\varepsilon_{j}}(\cdot, V)\right) .
$$


For all sufficiently large $j$, we also have $\eta_{j} \phi \in \mathcal{A}_{j}$, thus we may proceed as in (4.35) and estimate

$$
\begin{aligned}
\delta(V, \phi)\left(\eta_{j} h_{\varepsilon_{j}}(\cdot, V)\right)= & \delta V\left(\phi \eta_{j} h_{\varepsilon_{j}}\right)+\int_{\mathbf{G}_{n\left(\mathbb{R}^{n+1}\right)}} \eta_{j} h_{\varepsilon_{j}}(I-S)(\nabla \phi) d V(x, S) \\
\leq & -\left(1-\varepsilon_{j}^{1 / 4}\right) \int \eta_{j} \phi \frac{\left|\Phi_{\varepsilon_{j}} * \delta V\right|^{2}}{\Phi_{\varepsilon_{j}} *\|V\|+\varepsilon_{j}} d x \\
& +\varepsilon_{j}^{1 / 4}+\frac{1}{2} \int \eta_{j} \phi\left|h_{\varepsilon_{j}}\right|^{2} d\|V\| \\
& +\frac{1}{2} \int \frac{|S(\nabla \phi)|^{2}}{\phi} d V+\int h_{\varepsilon_{j}} \cdot \nabla \phi d\|V\| .
\end{aligned}
$$

Here we have used that $\eta_{j}=1$ when $\nabla \phi \neq 0$. In the present proof, we omit the domains of integration, which are either $\mathbb{R}^{n+1}$ or $\mathbf{G}_{n}\left(\mathbb{R}^{n+1}\right)$ unless specified otherwise. We use (3.34) to proceed as:

$$
\begin{aligned}
& \leq-\left(1-\frac{1}{2}-\frac{3 \varepsilon_{j}^{1 / 4}}{2}\right) \int \eta_{j} \phi \frac{\left|\Phi_{\varepsilon_{j}} * \delta V\right|^{2}}{\Phi_{\varepsilon_{j}} *\|V\|+\varepsilon_{j}} d x+2 \varepsilon_{j}^{1 / 4} \\
& +\frac{1}{2} \int \frac{|S(\nabla \phi)|^{2}}{\phi} d V+\int h_{\varepsilon_{j}} \cdot \nabla \phi d\|V\| .
\end{aligned}
$$

We prove that the last term gives a good negative contribution. We have

$$
\begin{aligned}
& \int h_{\varepsilon_{j}} \cdot \nabla \phi d\|V\|=-\int \Phi_{\varepsilon_{j}} * \frac{\Phi_{\varepsilon_{j}} * \delta V}{\Phi_{\varepsilon_{j}} *\|V\|+\varepsilon_{j}} \cdot \nabla \phi d\|V\| \\
& =-\int\left(\frac{\Phi_{\varepsilon_{j}} * \delta V}{\Phi_{\varepsilon_{j}} * V \|+\varepsilon_{j}}\right)(y) \cdot \int \Phi_{\varepsilon_{j}}(x-y) \nabla \phi(x) d\|V\|(x) d y .
\end{aligned}
$$

Here we replace $\nabla \phi(x)$ by $\nabla \phi(y)$ and estimate the error

$$
\left|\int\left(\frac{\Phi_{\varepsilon_{j}} * \delta V}{\Phi_{\varepsilon_{j}} *\|V\|+\varepsilon_{j}}\right)(y) \cdot \int \Phi_{\varepsilon_{j}}(x-y)(\nabla \phi(x)-\nabla \phi(y)) d\|V\|(x) d y\right| .
$$

To estimate (6.7), since $\eta_{j} \phi \in \mathcal{A}_{j}$, (3.1) and (3.3) imply

$$
|\nabla \phi(x)-\nabla \phi(y)|=\left|\nabla\left(\eta_{j} \phi\right)(x)-\nabla\left(\eta_{j} \phi\right)(y)\right| \leq j|x-y| \eta_{j}(y) \phi(y) \exp (j|x-y|) .
$$

By separating the integration to $B_{\sqrt{\varepsilon_{j}}}(y)$ and $B_{1}(y) \backslash B_{\sqrt{\varepsilon_{j}}}(y)$,

$$
\begin{aligned}
\int & \Phi_{\varepsilon_{j}}(x-y)|\nabla \phi(x)-\nabla \phi(y)| d\|V\|(x) \\
\leq & j \sqrt{\varepsilon_{j}} \exp \left(j \sqrt{\varepsilon_{j}}\right) \eta_{j}(y) \phi(y)\left(\Phi_{\varepsilon_{j}} *\|V\|\right)(y) \\
+ & c(n) \varepsilon_{j}^{-n-1} j \exp \left(j-\left(2 \varepsilon_{j}\right)^{-1}\right) \eta_{j}(y) \phi(y)\|V\|\left(B_{1}(y)\right) .
\end{aligned}
$$

Let us denote $c_{\varepsilon_{j}}:=c(n) \varepsilon_{j}^{-n-1} j \exp \left(j-\left(2 \varepsilon_{j}\right)^{-1}\right)$ and note that it is exponentially small (say, $\leq \exp \left(-\varepsilon_{j}^{-1 / 2}\right)$ for all large $\left.j\right)$ due to $j \leq \varepsilon_{j}^{-1 / 6} / 2$. Similarly we have $j \sqrt{\varepsilon_{j}} \exp \left(j \sqrt{\varepsilon_{j}}\right) \leq \varepsilon_{j}^{1 / 4}$, so that

$$
\begin{aligned}
& \int \Phi_{\varepsilon_{j}}(x-y)|\nabla \phi(x)-\nabla \phi(y)| d\|V\|(x) \\
& \leq\left(\varepsilon_{j}^{1 / 4}\left(\Phi_{\varepsilon_{j}} *\|V\|\right)(y)+c_{\varepsilon_{j}}\|V\|\left(B_{1}(y)\right)\right) \eta_{j}(y) \phi(y) .
\end{aligned}
$$


Using this, we can estimate

$$
\begin{aligned}
|(6.7)| & \leq\left(\int \eta_{j} \phi \frac{\left|\Phi_{\varepsilon_{j}} * \delta V\right|^{2}}{\Phi_{\varepsilon_{j}} *\|V\|+\varepsilon_{j}}\right)^{\frac{1}{2}}\left(2 \int \varepsilon_{j}^{\frac{1}{2}}\left(\Phi_{\varepsilon_{j}} *\|V\|\right)(y)+c_{\varepsilon_{j}}^{2} \varepsilon_{j}^{-1}\|V\|\left(B_{1}(y)\right)^{2} d y\right)^{\frac{1}{2}} \\
& \leq \varepsilon_{j}^{\frac{1}{4}} \int \eta_{j} \phi \frac{\left|\Phi_{\varepsilon_{j}} * \delta V\right|^{2}}{\Phi_{\varepsilon_{j}} *\|V\|+\varepsilon_{j}}+\int \varepsilon_{j}^{\frac{1}{4}}\left(\Phi_{\varepsilon_{j}} *\|V\|\right)(y)+c_{\varepsilon_{j}}^{2} \varepsilon_{j}^{-\frac{5}{4}}\|V\|\left(B_{1}(y)\right)^{2} d y .
\end{aligned}
$$

In view of (6.5), this shows that (6.7) can be absorbed as a small error term. Continuing from (6.6) with $\nabla \phi(y)$ replacing $\nabla \phi(x)$,

$$
\begin{aligned}
& -\int\left(\frac{\Phi_{\varepsilon_{j}} * \delta V}{\Phi_{\varepsilon_{j}} *\|V\|+\varepsilon_{j}}\right)(y) \cdot \int \Phi_{\varepsilon_{j}}(x-y) \nabla \phi(y) d\|V\|(x) d y \\
& =-\int\left(\frac{\Phi_{\varepsilon_{j}} * \delta V}{\Phi_{\varepsilon_{j}} *\|V\|+\varepsilon_{j}}\right)(y) \cdot \nabla \phi(y)\left(\Phi_{\varepsilon_{j}} *\|V\|\right)(y) d y \\
& =-\int\left(\Phi_{\varepsilon_{j}} * \delta V\right) \cdot \nabla \phi d y+\varepsilon_{j} \int\left(\frac{\Phi_{\varepsilon_{j}} * \delta V}{\Phi_{\varepsilon_{j}} * V \|+\varepsilon_{j}}\right)(y) \cdot \nabla \phi(y) d y .
\end{aligned}
$$

The last term of (6.10) may be estimated as

$$
\begin{aligned}
& \varepsilon_{j}\left|\int\left(\frac{\Phi_{\varepsilon_{j}} * \delta V}{\Phi_{\varepsilon_{j}} *\|V\|+\varepsilon_{j}}\right)(y) \cdot \nabla \phi(y) d y\right| \leq j \varepsilon_{j} \int_{(U)_{2}} \eta_{j} \phi \frac{\left|\Phi_{\varepsilon_{j}} * \delta V\right|}{\Phi_{\varepsilon_{j}} *\|V\|+\varepsilon_{j}} \\
& \quad \leq j \varepsilon_{j}^{\frac{1}{2}}\left(\int \eta_{j} \phi \frac{\left|\Phi_{\varepsilon_{j}} * \delta V\right|^{2}}{\Phi_{\varepsilon_{j}} *\|V\|+\varepsilon_{j}}\right)^{\frac{1}{2}}\left(\int_{(U)_{2}} \eta_{j} \phi\right)^{\frac{1}{2}} \\
& \quad \leq \varepsilon_{j}^{\frac{1}{4}} \int \eta_{j} \phi \frac{\left|\Phi_{\varepsilon_{j}} * \delta V\right|^{2}}{\Phi_{\varepsilon_{j}} *\|V\|+\varepsilon_{j}}+j^{2} \varepsilon_{j}^{\frac{3}{4}} \int_{(U)_{2}} \eta_{j} \phi .
\end{aligned}
$$

Here, we used the fact that the integrand is 0 far away from $U$, for example, outside of $(U)_{2}$. The last term of (6.11) can be absorbed as a small error since $j \leq \varepsilon_{j}^{-1 / 6} / 2$ and $\int_{(U)_{2}} \eta_{j} \phi$ is bounded by a constant. We can continue as

$$
\begin{aligned}
-\int\left(\Phi_{\varepsilon_{j}} * \delta V\right) \cdot \nabla \phi d y & =-\iint S\left(\nabla \Phi_{\varepsilon_{j}}(x-y)\right) d V(x, S) \nabla \phi(y) d y \\
& =-\int S \cdot\left(\int \nabla \Phi_{\varepsilon_{j}}(x-y) \otimes \nabla \phi(y) d y\right) d V(x, S) \\
& =-\int S \cdot \int \Phi_{\varepsilon_{j}}(x-y) \nabla^{2} \phi(y) d y d V(x, S) .
\end{aligned}
$$

We replace $\nabla^{2} \phi(y)$ by $\nabla^{2} \phi(x)$, with the resulting error being estimated, for instance, by $\leq M \varepsilon_{j}^{1 / 2}$ using standard methods as above. Then, we have

$$
-\int\left(\Phi_{\varepsilon_{j}} * \delta V\right) \cdot \nabla \phi d y \leq-\int S \cdot \nabla^{2} \phi(x) d V(x, S)+M \varepsilon_{j}^{1 / 2} .
$$

Thus, combining (6.4)-(6.12) and recovering the notations, we obtain

$$
\frac{\left\|\partial \mathcal{E}_{j, k}\right\|(\phi)-\left\|\partial \mathcal{E}_{j, k-1}\right\|(\phi)}{\Delta t_{j}} \leq 2 \varepsilon_{j}^{1 / 8}+\int \frac{|S(\nabla \phi)|^{2}}{2 \phi}-S \cdot \nabla^{2} \phi d V
$$


for all sufficiently large $j$. By (6.3), we have

$$
\begin{aligned}
\frac{|S(\nabla \phi)|^{2}}{2 \phi}-S \cdot \nabla^{2} \phi & =\left(\frac{\beta^{2}}{2} \sum_{i=1}^{n+1} S_{i, n+1}^{2}-\beta(\beta-1) S_{n+1, n+1}\right) d_{A}^{\beta-2} \\
& =\left(\frac{\beta^{2}}{2}-\beta(\beta-1)\right)\left|S_{n+1, n+1}\right| d_{A}^{\beta-2},
\end{aligned}
$$

where in the last identity we have used that $S$ is the matrix representing an orthogonal projection operator, so that $S$ is symmetric and $S^{2}=S$, whence

$$
S_{n+1, n+1}=\left(S^{2}\right)_{n+1, n+1}=\sum_{i=1}^{n+1} S_{i, n+1}^{2} \geq 0 .
$$

In particular, the quantity in (6.14) can be made negative if $\beta=4$, for example. This shows that (6.13) is less than $2 \varepsilon_{j}^{1 / 8}$. By summing over $k=1, \ldots, j^{1 / 2} /\left(\Delta t_{j}\right)$ and using that $\left\|\partial \mathcal{E}_{j, 0}\right\|(\phi)=s \mathcal{H}^{n}\left(\Gamma_{0}\right)$, we obtain

$$
\sup _{t \in\left[0, j^{1 / 2}\right]}\left\|\partial \mathcal{E}_{j}(t)\right\|(\phi) \leq 2 \varepsilon_{j}^{1 / 8} j^{1 / 2}+s \mathcal{H}^{n}\left(\Gamma_{0}\right) .
$$

Fix $\rho>0$ so that $C \subset\left\{x_{n+1}>\rho\right\}$. Then we have $\phi \geq \rho^{\beta}$ on $C$. With this, we have $\left\|\partial \mathcal{E}_{j}(t)\right\|(C) \leq \rho^{-\beta}\left\|\partial \mathcal{E}_{j}(t)\right\|(\phi)$. We use this in (6.15), and we let first $j \rightarrow \infty$ and then $s \rightarrow 0$ in order to obtain (6.1).

Proposition 6.4 For all $t \geq 0$, we have spt $\left\|V_{t}\right\| \subset \operatorname{conv}\left(\Gamma_{0} \cup \partial \Gamma_{0}\right)$.

Proof Suppose that $A \subset \mathbb{R}^{n+1}$ is a hyperplane such that, using the notation in the statement of Proposition 6.1, $\Gamma_{0} \cup \partial \Gamma_{0} \subset A^{-}$. If $d_{A}$ is $v_{U}$-non decreasing in $A^{+}$, then (6.1) proves immediately that $\left\|V_{t}\right\|\left(A^{+}\right)=0$ for all $t \geq 0$. Thus, suppose that $d_{A}$ does not satisfy this property. Still, due to Proposition 6.1, for each $x \in \partial U \backslash \partial \Gamma_{0}$, there exists a neighborhood $B_{r}(x)$ such that $\left\|V_{t}\right\|\left(B_{r}(x) \cap U\right)=0$ for all $t \geq 0$. In particular, there exists some $r_{0}>0$ such that

$$
\left\|V_{t}\right\|\left((\partial U)_{r_{0}} \cap A^{+}\right)=0
$$

for all $t \geq 0$. Let $\psi \in C_{c}^{\infty}\left(U ; \mathbb{R}^{+}\right)$be such that $\psi=1$ on $U \backslash(\partial U)_{r_{0}}$ and $\psi=0$ on $(\partial U) \frac{r_{0}}{2}$. We next use $\phi=\psi d_{A}^{4}$ in (5.8) with $t_{1}=0$ and an arbitrary $t_{2}=t>0$ to obtain

$$
\begin{aligned}
\left.\left\|V_{s}\right\|(\phi)\right|_{s=0} ^{t} & \leq \int_{0}^{t} \int_{U}\left(\nabla \phi-\phi h\left(\cdot, V_{s}\right)\right) \cdot h\left(\cdot, V_{s}\right) d\left\|V_{s}\right\| d s \\
& \leq-\int_{0}^{t} \int_{U} S \cdot \nabla^{2} \phi d V_{s}(\cdot, S) d s .
\end{aligned}
$$

By (6.16), $\phi=d_{A}^{4}$ on the support of $\left\|V_{S}\right\|$. Since $S \cdot \nabla^{2} d_{A}^{4} \geq 0$ for any $S \in \mathbf{G}(n+1, n)$ (see (6.14)), the right-hand side of (6.17) is $\leq 0$. Since $\left\|V_{0}\right\|(\phi)=0$, we have $\left\|V_{t}\right\|\left(A^{+}\right)=0$ for all $t>0$. This proves the claim.

In the following, we list results from [20, Section 10]. The results are local in nature, thus even if we are concerned with a Brakke flow in $U$ instead of $\mathbb{R}^{n+1}$, the proofs are the same. We recall the following (cf. Theorem 2.3(11)): 
Definition 6.5 Define a Radon measure $\mu$ on $U \times \mathbb{R}^{+}$by setting $d \mu:=d\left\|V_{t}\right\| d t$, namely

$$
\begin{aligned}
& \int_{U \times \mathbb{R}^{+}} \phi(x, t) d \mu(x, t):=\int_{0}^{\infty}\left(\int_{U} \phi(x, t) d\left\|V_{t}\right\|(x)\right) d t \\
& \quad \text { for every } \phi \in C_{c}\left(U \times \mathbb{R}^{+}\right) .
\end{aligned}
$$

Lemma 6.6 We have the following properties for $\mu$ and $\left\{V_{t}\right\}_{t \in \mathbb{R}^{+}}$.

(1) $\operatorname{spt}\left\|V_{t}\right\| \subset\{x \in U:(x, t) \in \operatorname{spt} \mu\}$ for all $t>0$.

(2) For each $\tilde{U} \subset \subset U$ and $t>0$, we have $\mathcal{H}^{n}(\{x \in \tilde{U}:(x, t) \in \operatorname{spt} \mu\})<\infty$.

The next Lemma (see [20, Lemma 10.10 and 10.11]) is used to prove the continuity of the labeling of partitions.

Lemma 6.7 Let $\left\{\mathcal{E}_{j_{\ell}}(t)\right\}_{\ell=1}^{\infty}$ be the sequence obtained in Proposition 5.1, and let $\left\{E_{j_{\ell}, i}(t)\right\}_{i=1}^{N}$ denote the open partitions for each $j_{\ell}$ and $t \in \mathbb{R}^{+}$, i.e., $\mathcal{E}_{j_{\ell}}(t)=\left\{E_{j_{\ell}, i}(t)\right\}_{i=1}^{N}$.

(1) For fixed $i \in\{1, \ldots, N\}, B_{2 r}(x) \subset \subset U, t>0$ with $t-r^{2}>0$, suppose that

$$
\lim _{\ell \rightarrow \infty} \mathcal{L}^{n+1}\left(B_{2 r}(x) \backslash E_{j_{\ell}, i}(t)\right)=0 \text { and } \mu\left(B_{2 r}(x) \times\left[t-r^{2}, t+r^{2}\right]\right)=0 .
$$

Then for all $t^{\prime} \in\left(t-r^{2}, t+r^{2}\right]$, we have

$$
\lim _{\ell \rightarrow \infty} \mathcal{L}^{n+1}\left(B_{r}(x) \backslash E_{j \ell, i}\left(t^{\prime}\right)\right)=0 .
$$

(2) For fixed $i \in\{1, \ldots, N\}, B_{2 r}(x) \subset \subset U$ and $r>0$, suppose that

$$
B_{2 r}(x) \subset E_{j_{\ell}, i}(0) \quad \text { for all } \ell \in \mathbb{N} \text { and } \mu\left(B_{2 r}(x) \times\left[0, r^{2}\right]\right)=0 .
$$

Then for all $t^{\prime} \in\left(0, r^{2}\right]$, we have

$$
\lim _{\ell \rightarrow \infty} \mathcal{L}^{n+1}\left(B_{r}(x) \backslash E_{j \ell, i}\left(t^{\prime}\right)\right)=0 .
$$

The following is from $[2,3.7]$.

Lemma 6.8 Suppose that $\left\|V_{t}\right\|\left(U_{r}(x)\right)=0$ for some $t \in \mathbb{R}^{+}$and $U_{r}(x) \subset \subset U$. Then, for every $t^{\prime} \in\left[t, t+\frac{r^{2}}{2 n}\right]$ it holds $\left\|V_{t^{\prime}}\right\|\left(U_{\sqrt{r^{2}-2 n\left(t^{\prime}-t\right)}}(x)\right)=0$.

Proof of Theorem 2.3 Let $\left\{\mathcal{E}_{j_{\ell}}(t)\right\}_{\ell=1}^{\infty}$ be a sequence as in Lemma 6.7, with $\mathcal{E}_{j_{\ell}}(t)=$ $\left\{E_{j \ell, i}(t)\right\}_{i=1}^{N}$ for every $\ell \in \mathbb{N}$. Since $E_{j_{\ell}, i}(t) \subset(U)_{1}$, for each $t$ and $i$ the volumes $\mathcal{L}^{n+1}\left(E_{j_{\ell}, i}(t)\right)$ are uniformly bounded in $\ell$. Furthermore, by the mass estimate in (4.31) we also have that $\left\|\nabla \chi_{E_{j_{\ell}, i}(t)}\right\|\left(\mathbb{R}^{n+1}\right)$ are uniformly bounded. Hence, we can use the compactness theorem for sets of finite perimeter in order to select a (not relabeled) subsequence with the property that, for each fixed $i \in\{1, \ldots, N\}$,

$$
\chi_{E_{j_{\ell}, i}(t)} \rightarrow \chi_{E_{i}(t)} \text { in } L_{l o c}^{1}\left(\mathbb{R}^{n+1}\right) \quad \text { for every } t \in 2_{\mathbb{Q}},
$$

where $E_{i}(t)$ is a set of locally finite perimeter in $\mathbb{R}^{n+1}$. Moreover, using that $E_{j \ell, i}(t) \subset$ $(U)_{1 /\left(4 j_{\ell}^{1 / 4}\right)}$ (see Proposition 4.2 and (4.7)) we see that $\mathcal{L}^{n+1}\left(E_{i}(t) \backslash U\right)=0$. Since sets of finite perimeter are defined up to measure zero sets, we can then assume without loss of generality that $E_{i}(t) \subset U$. Hence, since $\mathcal{H}^{n}(\partial U)<\infty, E_{i}(t)$ is in fact a set of finite perimeter in $\mathbb{R}^{n+1}$.

Next, consider the complement of spt $\mu \cup\left(\Gamma_{0} \times\{0\}\right)$ in $U \times \mathbb{R}^{+}$, which is relatively open in $U \times \mathbb{R}^{+}$, and let $S$ be one of its connected components. For any point $(x, t) \in S$ there 
exists $r>0$ such that either $B_{2 r}(x) \times\left[t-r^{2}, t+r^{2}\right] \subset S$ if $t>0$, or $B_{2 r}(x) \times\left[0, r^{2}\right] \subset S$ if $t=0$. We first consider the case $t=0$. Since $B_{2 r}(x)$ lies in the complement of $\Gamma_{0}$, there exists $i(x, 0) \in\{1, \ldots, N\}$ such that $B_{2 r}(x) \subset E_{0, i(x, 0)}$, and thus $B_{2 r}(x) \subset E_{j_{\ell}, i(x, 0)}(0)$ for all $\ell \in \mathbb{N}$. Since also $\mu\left(B_{2 r}(x) \times\left[0, r^{2}\right]\right)=0$, we can apply Lemma 6.7(2) and conclude that

$$
\lim _{\ell \rightarrow \infty} \mathcal{L}^{n+1}\left(B_{r}(x) \backslash E_{j \ell, i(x, 0)}\left(t^{\prime}\right)\right)=0 \quad \text { for all } t^{\prime} \in\left(0, r^{2}\right] .
$$

Similarly, if $t>0$, since $\mu\left(B_{2 r}(x) \times\left[t-r^{2}, t+r^{2}\right]\right)=0$, we can apply Lemma 6.7(1) to conclude that there is a unique $i(x, t) \in\{1, \ldots, N\}$ such that

$$
\lim _{\ell \rightarrow \infty} \mathcal{L}^{n+1}\left(B_{r}(x) \backslash E_{j \ell, i(x, t)}\left(t^{\prime}\right)\right)=0 \quad \text { for all } t^{\prime} \in\left(t-r^{2}, t+r^{2}\right] .
$$

Now, observe that if $S$ is any connected component of the complement of spt $\mu \cup\left(\Gamma_{0} \times\{0\}\right)$ in $U \times \mathbb{R}^{+}$, then by (6.20) and (6.21), and since $S$ is connected, for any two points $(x, t)$ and $(y, s)$ in $S$ it has to be $i(x, t)=i(y, s)$. For every $i \in\{1, \ldots, N\}$, we can then let $S(i)$ denote the union of all connected components $S$ such that $i(x, t)=i$ for every $(x, t) \in S$. It is clear that $S(i)$ are open sets, and that $E_{0, i}=\{x \in U:(x, 0) \in S(i)\}$ (notice that if $x \in E_{0, i}$ then $(x, 0) \notin$ spt $\mu$ as a consequence of Lemma 6.8), so that each $S(i)$ is not empty. Furthermore, we have that $\bigcup_{i=1}^{N} S(i)=\left(U \times \mathbb{R}^{+}\right) \backslash\left(\operatorname{spt} \mu \cup\left(\Gamma_{0} \times\{0\}\right)\right)$. For every $t \in \mathbb{R}^{+}$, we can thus define

$$
E_{i}(t):=\{x \in U:(x, t) \in S(i)\}, \Gamma(t):=U \backslash \cup_{i=1}^{N} E_{i}(t) .
$$

By examining the definition, one obtains $\Gamma(t)=\{x \in U:(x, t) \in \operatorname{spt} \mu\}$ for all $t>0$. Combined with Lemma 6.6(1), we have (11). By Lemma 6.6(2), we have (3), and this also proves that $\Gamma(t)$ has empty interior, which shows (4). The claims (1) and (2) hold true by construction. (5) is a consequence of Proposition 6.4 and the definition of $\mu$ being the product measure. (6) is similar: if $x \in U \backslash \operatorname{conv}\left(\Gamma_{0} \cup \partial \Gamma_{0}\right)$ then the half-line $t \in$ $\mathbb{R}^{+} \mapsto \gamma_{x}(t):=(x, t) \in U \times \mathbb{R}^{+}$must be contained in the same connected component of $\left(U \times \mathbb{R}^{+}\right) \backslash\left(\operatorname{spt} \mu \cup\left(\Gamma_{0} \times\{0\}\right)\right)$, for otherwise there would be $t>0$ such that $(x, t) \in \operatorname{spt} \mu$, thus contradicting (5). For (7), by the strict convexity of $U$ and (5), we have $\partial \Gamma(t) \subset \partial \Gamma_{0}$ for all $t>0$. Later in Proposition 6.9, we prove $\left(\operatorname{clos}\left(\operatorname{spt}\left\|V_{t}\right\|\right)\right) \backslash U=\partial \Gamma_{0}$ and $\partial \Gamma_{0} \subset \partial \Gamma(t)$ follows from this and (11). Coming to (8), we use (6.21) together with the conclusions in Proposition 4.2(1) to see that $\chi_{E_{j_{\ell}, i}(t)} \rightarrow \chi_{E_{i}(t)}$ in $L^{1}\left(\mathbb{R}^{n+1}\right)$ as $\ell \uparrow \infty$, for every $t \in \mathbb{R}^{+}$. In particular, the lower semi-continuity of perimeter allows us to deduce that for any $\phi \in$ $C_{c}\left(U ; \mathbb{R}^{+}\right)$

$$
\left\|\nabla \chi_{E_{i}(t)}\right\|(\phi) \leq \liminf _{\ell \rightarrow \infty}\left\|\nabla \chi_{E_{j_{\ell}, i}(t)}\right\|(\phi) \leq \liminf _{\ell \rightarrow \infty}\left\|\partial \mathcal{E}_{j_{\ell}}(t)\right\|(\phi)=\left\|V_{t}\right\|(\phi),
$$

thus proving $\left\|\nabla \chi_{E_{i}(t)}\right\| \leq\left\|V_{t}\right\|$ of (8). Using the cluster structure of each $\partial \mathcal{E}_{j_{\ell}}(t)$ (see e.g. [26, Proposition 29.4]), we have in fact that

$\frac{1}{2} \sum_{i=1}^{N}\left\|\nabla \chi_{E_{j_{\ell}, i}(t)}\right\|(\phi)=\mathcal{H}^{n}\left\llcorner_{\left(\cup_{i=1}^{N} \partial^{*} E_{j_{\ell}, i}(t)\right)}(\phi) \leq\left\|\partial \mathcal{E}_{j_{\ell}}(t)\right\|(\phi) \quad\right.$ for every $\phi$ as above,

which shows the other statement $\sum_{i=1}^{N}\left\|\nabla \chi_{E_{i}(t)}\right\| \leq 2\left\|V_{t}\right\|$ in (8). Since the claim of (9) is interior in nature, the proof is identical to the case without boundary as in [20, Theorem 3.5(6)]. For the proof of (10), for $\bar{t} \geq 0$, we prove that $\chi_{E_{i}(t)} \rightarrow \chi_{E_{i}(\bar{t})}$ in $L^{1}(U)$ as $t \rightarrow \bar{t}$ for each $i=1, \ldots, N$. Since $\left\|\nabla \chi_{E_{i}(t)}\right\|(U) \leq\left\|V_{t}\right\|(U) \leq \mathcal{H}^{n}\left(\Gamma_{0}\right)$, for any $t_{k} \rightarrow \bar{t}$, there exists a subsequence (denoted by the same index) and $\tilde{E}_{i} \subset U$ such that $\chi_{E_{i}\left(t_{k}\right)} \rightarrow \chi_{\tilde{E}_{i}}$ 
in $L^{1}(U)$ and $\mathcal{L}^{n+1}$ a.e. by the compactness theorem for sets of finite perimeter. We also have $\mathcal{L}^{n+1}\left(\tilde{E}_{i} \cap \tilde{E}_{j}\right)=0$ for $i \neq j$ and $\mathcal{L}^{n+1}\left(U \backslash \cup_{i=1}^{N} \tilde{E}_{i}\right)=0$. For a contradiction, assume that $\mathcal{L}^{n+1}\left(E_{i}(\bar{t}) \backslash \tilde{E}_{i}\right)>0$ for some $i$. Then, there must be $U_{r}(x) \subset \subset E_{i}(\bar{t})$ such that $\mathcal{L}^{n+1}\left(U_{r}(x) \backslash \tilde{E}_{i}\right)>0$. We then use Theorem 2.3(9) with $g(t)=\mathcal{L}^{n+1}\left(E_{i}(t) \cap U_{r}(x)\right)$, which gives $\lim _{t \rightarrow \bar{t}} g(t)=g(\bar{t})=\mathcal{L}^{n+1}\left(E_{i}(\bar{t}) \cap U_{r}(x)\right)=\mathcal{L}^{n+1}\left(U_{r}(x)\right)$. On the other hand, $\chi_{E_{i}(t)} \rightarrow \chi_{\tilde{E}_{i}}$ in $L^{1}(U)$ implies $\lim _{t \rightarrow \bar{t}} g(t)=\mathcal{L}^{n+1}\left(\tilde{E}_{i} \cap U_{r}(x)\right)<\mathcal{L}^{n+1}\left(U_{r}(x)\right)$ because of $\mathcal{L}^{n+1}\left(U_{r}(x) \backslash \tilde{E}_{i}\right)>0$. This is a contradiction. Thus, we have $\mathcal{L}^{n+1}\left(E_{i}(\bar{t}) \backslash \tilde{E}_{i}\right)=0$ for all $i=1, \ldots, N$. Since $\left\{\tilde{E}_{1}, \ldots, \tilde{E}_{N}\right\}$ is a partion of $U$, we have $\mathcal{L}^{n+1}\left(E_{i}(\bar{t}) \triangle \tilde{E}_{i}\right)=0$ for all $i$. This proves (9), and finishes the proof of (1)-(11) except for (7), which is independent and is proved once we prove Proposition 6.9.

Proposition 6.9 For all $t \geq 0$, it holds $\left(\operatorname{clos}\left(\operatorname{spt}\left\|V_{t}\right\|\right)\right) \backslash U=\partial \Gamma_{0}$.

Proof Let $x \in\left(\operatorname{clos}\left(\operatorname{spt}\left\|V_{t}\right\|\right)\right) \backslash U$, and let $\left\{x_{k}\right\}_{k=1}^{\infty}$ be a sequence with $x_{k} \in \operatorname{spt}\left\|V_{t}\right\|$ such that $x_{k} \rightarrow x$ as $k \uparrow \infty$. If $x \notin \partial \Gamma_{0}$, then by Proposition 6.1 there is $r>0$ such that $\left\|V_{t}\right\|\left(B_{r}(x) \cap U\right)=0$. For all suitably large $k$ so that $\left|x-x_{k}\right|<r$ we then have $\left\|V_{t}\right\|\left(B_{r-\left|x-x_{k}\right|}\left(x_{k}\right) \cap U\right)=0$, which contradicts the fact that $x_{k} \in \operatorname{spt}\left\|V_{t}\right\|$.

Conversely, let $x \in \partial \Gamma_{0}$, and suppose for a contradiction that $x \notin \operatorname{clos}\left(\operatorname{spt}\left\|V_{t}\right\|\right.$ ), so that there is a radius $r>0$ with the property that $B_{r}(x) \cap \operatorname{spt}\left\|V_{t}\right\|=\emptyset$. Then, Theorem 2.3(8) implies that $\left\|\nabla \chi_{E_{i}(t)}\right\|\left(B_{r}(x) \cap U\right)=0$ for every $i \in\{1, \ldots, N\}$. Since $B_{r}(x) \cap U$ is connected by the convexity of $U$, every $\chi_{E_{i}(t)}$ is either constantly equal to 0 or 1 on $B_{r}(x) \cap U$, namely

$$
B_{r}(x) \cap U \subset E_{\ell}(t) \quad \text { for some } \ell \in\{1, \ldots, N\} .
$$

If $t=0$, since $E_{i}(0)=E_{0, i}$ for every $i=1, \ldots, N$, the conclusion in (6.23) is evidently incompatible with (A4), thus providing the desired contradiction. We can then assume $t>$ 0 . By $(A 4)$, there are at least two indices $i \neq i^{\prime} \in\{1, \ldots, N\}$ and sequences of balls $\left\{B_{r_{j}}\left(x_{j}\right)\right\}_{j=1}^{\infty},\left\{B_{r_{j}^{\prime}}\left(x_{j}^{\prime}\right)\right\}_{j=1}^{\infty}$ such that $x_{j}, x_{j}^{\prime} \in \partial U, \lim _{j \rightarrow \infty} x_{j}=\lim _{j \rightarrow \infty} x_{j}^{\prime}=x$ and $B_{r_{j}}\left(x_{j}\right) \cap U \subset E_{0, i}$ whereas $B_{r_{j}^{\prime}}\left(x_{j}^{\prime}\right) \cap U \subset E_{0, i^{\prime}}$. Let $z$ denote any of the points $x_{j}$ or $x_{j}^{\prime}$, and observe that the above condition guarantees that $z \in \partial U \backslash \partial \Gamma_{0}$. In turn, by arguing as in Remark 6.2 we deduce that there is a neighborhood $B_{\rho}(z) \cap U$ such that $\left\|V_{t}\right\|\left(B_{\rho}(z) \cap U\right)=0$ for all $t \geq 0$, and thus also $\left\|\nabla \chi_{E_{l}(t)}\right\|\left(B_{\rho}(z) \cap U\right)=0$ for every $t \geq 0$ and for every $l \in\{1, \ldots, N\}$. Since $B_{\rho}(z) \cap U$ is connected this implies that $B_{\rho}(z) \cap U \subset E_{l}(t)$ for some $l$. Applying this argument with $z=x_{j}$ and $z=x_{j}^{\prime}$ we then find radii $\rho_{j}$ and $\rho_{j}^{\prime}$ such that, necessarily, $B_{\rho_{j}}\left(x_{j}\right) \cap U \subset E_{i}(t)$ whereas $B_{\rho_{j}^{\prime}}\left(x_{j}^{\prime}\right) \cap U \subset E_{i^{\prime}}(t)$ for all $t \geq 0$. Since $x_{j} \rightarrow x$ and $x_{j}^{\prime} \rightarrow x$ this conclusion is again incompatible with (6.23), thus completing the proof.

Proposition 6.10 We have for each $\phi \in C_{c}\left(U ; \mathbb{R}^{+}\right)$

$$
\mathcal{H}^{n}\left\llcorner_{\left(\cup_{i=1}^{N} \partial^{*} E_{0, i}\right)}(\phi) \leq \liminf _{t \downarrow 0}\left\|V_{t}\right\|(\phi)=\limsup _{t \downarrow 0}\left\|V_{t}\right\|(\phi) \leq \mathcal{H}^{n} L_{\Gamma_{0}}(\phi) .\right.
$$

In particular, if $\mathcal{H}^{n}\left(\Gamma_{0} \backslash \cup_{i=1}^{N} \partial^{*} E_{0, i}\right)=0$, then we have

$$
\lim _{t \downarrow 0}\left\|V_{t}\right\|=\mathcal{H}^{n}\left\llcorner_{\Gamma_{0}} \quad \text { as Radon measures in } U .\right.
$$


Proof By [26, Proposition 29.4], we have for each $\phi \in C_{c}\left(U ; \mathbb{R}^{+}\right)$

$$
\begin{aligned}
& 2 \mathcal{H}^{n} L_{\left(\cup_{i=1}^{N} \partial^{*} E_{0, i}\right)}(\phi)=\sum_{i=1}^{N}\left\|\nabla \chi_{E_{0, i}}\right\|(\phi) \leq \sum_{i=1}^{N} \liminf _{t \downarrow 0}\left\|\nabla \chi_{E_{i}(t)}\right\|(\phi) \\
& \quad \leq \liminf _{t \downarrow 0} \sum_{i=1}^{N}\left\|\nabla \chi_{E_{i}(t)}\right\|(\phi) \leq 2 \liminf _{t \downarrow 0}\left\|V_{t}\right\|(\phi)
\end{aligned}
$$

where we also used Theorem 2.3(8) and (10). This proves the first inequality. The second equality and the third inequality follow from (4.28), $\mu_{t}=\left\|V_{t}\right\|$ and $\left\|V_{0}\right\|=\mathcal{H}^{n}\left\llcorner_{\Gamma_{0}}\right.$.

The proof of Theorem 2.2 is now complete: $\left\{V_{t}\right\}_{t \geq 0}$ is a Brakke flow with fixed boundary $\partial \Gamma_{0}$ due to Proposition 5.1(1), Theorem 5.4 and Proposition 6.9. Proposition 6.10 proves the claim on the continuity of measure at $t=0$.

\section{Applications to the problem of Plateau}

As anticipated in the introduction, an interesting byproduct of our global existence result for Brakke flow is the existence of a stationary integral varifold $V$ in $U$ satisfying the topological boundary constraint $\operatorname{clos}(\operatorname{spt}\|V\|) \backslash U=\partial \Gamma_{0}$. This is the content of Corollary 2.4, which we prove next.

Proof of Corollary 2.4 By the estimate in (5.7), the function

$$
H(t):=\int_{U}\left|h\left(x, V_{t}\right)\right|^{2} d\left\|V_{t}\right\|(x)
$$

is in $L^{1}((0, \infty))$. Hence, there exists a sequence $\left\{t_{k}\right\}_{k=1}^{\infty}$ such that

$$
\lim _{k \rightarrow \infty} t_{k}=\infty, \quad \lim _{k \rightarrow \infty} H\left(t_{k}\right)=0 .
$$

Let $V_{k}:=V_{t_{k}}$. Again by (5.7), we have that

$$
\sup _{k}\left\|V_{k}\right\|(U) \leq \mathcal{H}^{n}\left(\Gamma_{0}\right) .
$$

Furthermore, combining (2.5) with (7.2) yields, via the Cauchy-Schwarz inequality, that

$$
\left|\delta V_{k}(g)\right| \leq\|g\|_{C^{0}}\left(\mathcal{H}^{n}\left(\Gamma_{0}\right)\right)^{\frac{1}{2}}\left(H\left(t_{k}\right)\right)^{\frac{1}{2}} \quad \text { for every } g \in C_{c}\left(U ; \mathbb{R}^{n+1}\right),
$$

so that

$$
\lim _{k \rightarrow \infty}\left\|\delta V_{k}\right\|(U)=0 .
$$

Hence, we can apply Allard's compactness theorem for integral varifolds, see [33, Theorem 42.7], in order to conclude the existence of a stationary integral varifold $V \in \mathbf{I V}_{n}(U)$ such that $V_{k} \rightarrow V$ in the sense of varifolds.

Next, we prove the existence of the family $\left\{E_{i}\right\}_{i=1}^{N}$. Fix $i \in\{1, \ldots, N\}$, and consider the sequence $\left\{E_{i}^{k}\right\}_{k=1}^{\infty}$, where $E_{i}^{k}:=E_{i}\left(t_{k}\right)$. By Theorem 2.3(8) and (5.7) we have, along a (not relabeled) subsequence, the convergence

$$
\chi_{E_{i}^{k}} \rightarrow \chi_{E_{i}} \quad \text { in } L^{1}(U) \text { and pointwise } \mathcal{L}^{n+1} \text {-a.e. as } k \rightarrow \infty
$$


where $E_{i} \subset U$ are sets of finite perimeter. Since, by Theorem 2.3(3), $\sum_{i=1}^{N} \chi_{E_{i}^{k}}=\chi_{U}$ as $L^{1}$ functions, we conclude that

$$
\mathcal{L}^{n+1}\left(U \backslash \bigcup_{i=1}^{N} E_{i}\right)=0, \quad \text { and } \quad \mathcal{L}^{n+1}\left(E_{i} \cap E_{j}\right)=0 \text { if } i \neq j,
$$

so that $\bigcup_{i=1}^{N} E_{i}$ is an $\mathcal{L}^{n+1}$-partition of $U$. The validity of Theorem 2.3(8) implies conclusion (1), namely that

$$
\left\|\nabla \chi_{E_{i}}\right\| \leq\|V\| \text { for every } i \in\{1, \ldots, N\} \quad \text { and } \quad \sum_{i=1}^{N}\left\|\nabla \chi_{E_{i}}\right\| \leq 2\|V\|
$$

in the sense of Radon measures in $U$. As a consequence of (7.6), we have that spt $\left\|\nabla \chi_{E_{i}}\right\| \subset$ spt $\|V\|$ for every $i=1, \ldots, N$. Since $V$ is a stationary integral varifold, the monotonicity formula implies that $\operatorname{spt}\|V\|$ is $\mathcal{H}^{n}$-rectifiable, and $V=\operatorname{var}(\operatorname{spt}\|V\|, \theta)$ for some upper semi-continuous $\theta: U \rightarrow \mathbb{R}^{+}$with $\theta(x) \geq 1$ at each $x \in \operatorname{spt}\|V\|$. In particular, setting $\Gamma:=\operatorname{spt}\|V\|$, we have

$$
\mathcal{H}^{n}(\Gamma)=\|\operatorname{var}(\Gamma, 1)\|(U) \leq\|V\|(U) \leq \mathcal{H}^{n}\left(\Gamma_{0}\right),
$$

where the last inequality is a consequence of (5.7) and the lower semicontinuity of the weight with respect to varifold convergence.

Next, we observe that, since spt $\left\|\nabla \chi_{E_{i}}\right\| \subset \Gamma$, on each connected component of $U \backslash \Gamma$ each $\chi_{E_{i}}$ is almost everywhere constant. Denoting $\left\{O_{h}\right\}_{h \in \mathbb{N}}$ the connected components of the open set $U \backslash \Gamma$, we may then modify each set $E_{i}(i \in\{1, \ldots, N\})$ by setting

$$
\left.E_{i}^{*}:=\bigcup_{\left\{O_{h}: \chi_{E_{i}}=1\right.} O_{h} \text { a.e. on } O_{h}\right\}
$$

By definition, each set $E_{i}^{*}$ is open; furthermore, the sets $E_{i}^{*}$ are pairwise disjoint, and $\bigcup_{i=1}^{N} E_{i}^{*}=U \backslash \Gamma$. Since for each $i$ we have $\mathcal{L}^{n+1}\left(E_{i} \Delta E_{i}^{*}\right)=0$, and since sets of finite perimeter are defined up to $\mathcal{L}^{n+1}$-negligible sets, we can thus replace the family $\left\{E_{i}\right\}$ with $\left\{E_{i}^{*}\right\}$, and drop the superscript * to ease the notation.

Property (2) is a consequence of Theorem 2.3(6), since the convergence $\chi_{E_{i}^{k}} \rightarrow \chi_{E_{i}}$ now holds pointwise on $U \backslash \operatorname{conv}\left(\Gamma_{0} \cup \partial \Gamma_{0}\right)$. We have not excluded the possibility that $\mathcal{H}^{n}(\Gamma)=0$. But this should imply $\|V\|=0$ by (7.7), and $\left\|\nabla \chi_{E_{i}}\right\|=0$ for every $i \in\{1, \ldots, N\}$ by (7.6), which is a contradiction to (2). Thus we have necessarily $\mathcal{H}^{n}(\Gamma)>0$ and this completes the proof of (3). In order to conclude the proof, we are just left with the boundary condition (4), namely

$$
(\operatorname{clos}(\operatorname{spt}\|V\|)) \backslash U=\partial \Gamma_{0} .
$$

Towards the first inclusion, suppose that $x \in(\operatorname{clos}(\operatorname{spt}\|V\|)) \backslash U$, and let $\left\{x_{h}\right\}_{h=1}^{\infty}$ be a sequence with $x_{h} \in \operatorname{spt}\|V\|$ such that $x_{h} \rightarrow x$ as $h \rightarrow \infty$. If $x \notin \partial \Gamma_{0}$ then Proposition 6.1 implies that there exists $r>0$ such that

$$
\limsup _{k \rightarrow \infty}\left\|V_{k}\right\|\left(U \cap B_{r}(x)\right)=0 .
$$

By the lower semi-continuity of the weight with respect to varifold convergence, we deduce then that $\|V\|\left(U \cap U_{r}(x)\right)=0$. For $h$ large enough so that $\left|x-x_{h}\right|<r$ we then have $\|V\|\left(U \cap U_{r-\left|x-x_{h}\right|}\left(x_{h}\right)\right)=0$, thus contradicting that $x_{h} \in \mathrm{spt}\|V\|$. For the second inclusion, let $x \in \partial \Gamma_{0}$, and suppose towards a contradiction that $x \notin \operatorname{clos}(\operatorname{spt}\|V\|) \backslash U$. Then, there 
exists a radius $r>0$ such that $U_{r}(x) \cap$ spt $\|V\|=\emptyset$. In particular, $\left\|\nabla \chi_{E_{i}}\right\|\left(U \cap U_{r}(x)\right)=0$ for every $i \in\{1, \ldots, N\}$. Since $U$ is convex, $U \cap U_{r}(x)$ is connected, and thus every $\chi_{E_{i}}$ is either identically 0 or 1 in $U_{r}(x) \cap U$, namely

$$
U_{r}(x) \cap U \subset E_{\ell} \quad \text { for some } \ell \in\{1, \ldots, N\} .
$$

Because $x \in \partial \Gamma_{0}$, by property (A4) in Assumption 1.1 there are two indices $i \neq i^{\prime} \in$ $\{1, \ldots, N\}$ and sequences $\left\{x_{j}\right\}_{j=1}^{\infty},\left\{x_{j}^{\prime}\right\}_{j=1}^{\infty}$ with $\lim _{j \rightarrow \infty} x_{j}=x=\lim _{j \rightarrow \infty} x_{j}^{\prime}$ such that $x_{j}, x_{j}^{\prime} \in \partial U \backslash \partial \Gamma_{0}$ and $U_{r_{j}}\left(x_{j}\right) \cap U \subset E_{0, i}, U_{r_{j}^{\prime}}\left(x_{j}^{\prime}\right) \cap U \subset E_{0, i^{\prime}}$ for some $r_{j}, r_{j}^{\prime}>0$. If $z$ denotes any of the points $x_{j}$ or $x_{j}^{\prime}$, Proposition 6.1 and Remark 6.2 ensure the existence of $\rho$ such that $\left\|V_{t}\right\|\left(B_{\rho}(z) \cap U\right)=0$ for all $t \geq 0$. Again by lower semicontinuity of the weight with respect to varifold convergence, $\|V\|\left(U_{\rho}(z) \cap U\right)=0$. Since each $U_{\rho}(z) \cap U$ is connected and $\operatorname{spt}\left\|\nabla \chi_{E_{i}}\right\| \subset \operatorname{spt}\|V\|$ for all $i$, we deduce that $U_{\rho_{j}}\left(x_{j}\right) \cap U \subset E_{i}$ and $U_{\rho_{j}^{\prime}}\left(x_{j}^{\prime}\right) \cap U \subset E_{i^{\prime}}$ for some $i \neq i^{\prime}$. Since both $x_{j} \rightarrow x$ and $x_{j}^{\prime} \rightarrow x$, this conclusion is incompatible with (7.9). This completes the proof.

The stationary varifold $V$ from Corollary 2.4 is a generalized minimal surface in $U$, and for this reason it can be thought of as a solution to Plateau's problem in $U$ with the prescribed boundary $\partial \Gamma_{0}$. Brakke flow provides, therefore, an interesting alternative approach to the existence theory for Plateau's problem compared to more classical methods based on mass (or area) minimization. Another novelty of this approach is that the structure of partitions allows to prescribe the boundary datum in the purely topological sense, by means of the constraint (clos $(\operatorname{spt}\|V\|)) \backslash U=\partial \Gamma_{0}$. This adds to the several other possible interpretations of the spanning conditions that have been proposed in the literature: among them, let us mention the homological boundary conditions in Federer and Fleming's theory of integral currents [12] or of integral currents $\bmod (p)[11]$ (see also Brakke's covering space model for soap films [3]); the sliding boundary conditions in David's sliding minimizers [5,6]; and the homotopic spanning condition of Harrison [13], Harrison-Pugh [14] and De Lellis-GhiraldinMaggi [7].

Concerning the latter, we can actually show that, under a suitable extra assumption on the initial partition $\mathcal{E}_{0}$, a homotopic spanning condition is satisfied at all times along the flow. Before stating and proving this result, which is Proposition 7.4 below, let us first record the definition of homotopic spanning condition after [7].

Definition 7.1 (see [7, Definition 3]). Let $n \geq 2$, and let $\Sigma$ be a closed subset of $\mathbb{R}^{n+1}$. Consider the family

$$
\mathcal{C}_{\Sigma}:=\left\{\gamma: \mathbb{S}^{1} \rightarrow \mathbb{R}^{n+1} \backslash \Sigma: \gamma \text { is a smooth embedding of } \mathbb{S}^{1} \text { into } \mathbb{R}^{n+1} \backslash \Sigma\right\} .
$$

A subfamily $\mathcal{C} \subset \mathcal{C}_{\Sigma}$ is said to be homotopically closed if $\gamma \in \mathcal{C}$ implies that $\tilde{\gamma} \in \mathcal{C}$ for every $\tilde{\gamma} \in[\gamma]$, where $[\gamma]$ is the equivalence class of $\gamma$ modulo homotopies in $\mathbb{R}^{n+1} \backslash \Sigma$. Given a homotopically closed $\mathcal{C} \subset \mathcal{C}_{\Sigma}$, a relatively closed subset $K \subset \mathbb{R}^{n+1} \backslash \Sigma$ is $\mathcal{C}$-spanning $\Sigma$ if 2

$$
K \cap \gamma \neq \emptyset \quad \text { for every } \gamma \in \mathcal{C} \text {. }
$$

Remark 7.2 If $\mathcal{C} \subset \mathcal{C}_{\Sigma}$ contains a homotopically trivial curve, then any $\mathcal{C}$-spanning set $K$ will necessarily have non-empty interior (and therefore infinite $\mathcal{H}^{n}$ measure). For this reason, we are only interested in subfamilies $\mathcal{C}$ with $[\gamma] \neq 0$ for every $\gamma \in \mathcal{C}$.

${ }^{2}$ With a slight abuse of notation, in what follows we will always identify the map $\gamma$ with its image $\gamma\left(\mathbb{S}^{1}\right) \subset$ $\mathbb{R}^{n+1} \backslash \Sigma$. 
Definition 7.3 We will say that a relatively closed subset $K \subset \mathbb{R}^{n+1} \backslash \Sigma$ strongly homotopically spans $\Sigma$ if it $\mathcal{C}$-spans $\Sigma$ for every homotopically closed family $\mathcal{C} \subset \mathcal{C}_{\Sigma}$ which does not contain any homotopically trivial curve. Namely, if $K \cap \gamma \neq \emptyset$ for every $\gamma \in \mathcal{C}_{\Sigma}$ such that $[\gamma] \neq 0$ in $\pi_{1}\left(\mathbb{R}^{n+1} \backslash \Sigma\right)$.

We can prove the following proposition, whose proof is a suitable adaptation of the argument in [7, Lemma 10].

Proposition 7.4 Let $n \geq 2$, and let $U, \Gamma_{0}, \mathcal{E}_{0}$ be as in Assumption 1.1. Suppose that the initial partition $\mathcal{E}_{0}$ satisfies the following additional property:

Given any two connected components $S_{1}$ and $S_{2}$ of $\partial U \backslash \partial \Gamma_{0}$,

there are two indices $i, j \in\{1, \ldots, N\}$ with $i \neq j$

such that $S_{1} \subset \operatorname{clos} E_{0, i}$ and $S_{2} \subset \operatorname{clos} E_{0, j}$.

Then, the set $\Gamma(t)$ strongly homotopically spans $\partial \Gamma_{0}$ for every $t \in[0, \infty]$.

Proof Let $\gamma: \mathbb{S}^{1} \rightarrow \mathbb{R}^{n+1} \backslash \partial \Gamma_{0}$ be a smooth embedding that is not homotopically trivial in $\mathbb{R}^{n+1} \backslash \partial \Gamma_{0}$. The goal is to prove that, for every $t \in[0, \infty], \Gamma(t) \cap \gamma \neq \emptyset$. First observe that it cannot be $\gamma \subset U$, for otherwise $\gamma$ would be homotopically trivial. For the same reason, since the ambient dimension is $n+1 \geq 3$ also $\gamma \subset \mathbb{R}^{n+1} \backslash$ clos $U$ is incompatible with the properties of $\gamma$. Hence, we conclude that $\gamma$ must necessarily intersect $\partial U$. We first prove the result under the additional assumption that $\gamma$ and $\partial U$ intersect transversally. We can then find finitely many closed $\operatorname{arcs} I_{h}=\left[a_{h}, b_{h}\right] \subset \mathbb{S}^{1}$ with the property that $\gamma \cap U=\bigcup_{h} \gamma\left(\left(a_{h}, b_{h}\right)\right)$, and $\gamma \cap\left(\partial U \backslash \partial \Gamma_{0}\right)=\bigcup_{h}\left\{\gamma\left(a_{h}\right), \gamma\left(b_{h}\right)\right\}$. If there is $h$ such that $\gamma\left(a_{h}\right)$ and $\gamma\left(b_{h}\right)$ belong to two distinct connected components of $\partial U \backslash \partial \Gamma_{0}$, then $(\diamond)$ implies that the arc $\sigma_{h}:=\left.\gamma\right|_{\left(a_{h}, b_{h}\right)}$ must intersect $U \cap \partial E_{i}(0)$ for some $i=1, \ldots, N$. In fact, since the labeling of the open partition at the boundary of $U$ is invariant along the flow, the same conclusion holds for every $t \in[0, \infty]$. In particular, in this case $\gamma$ intersects $\bigcup_{i}\left(\partial E_{i}(t) \cap U\right)=\Gamma(t)$ for every $t \in[0, \infty]$. Hence, if by contradiction $\gamma$ has empty intersection with $\Gamma(t)$, then necessarily for every $h$ there is a connected component $S_{h}$ of $\partial U \backslash \partial \Gamma_{0}$ such that $\gamma\left(a_{h}\right), \gamma\left(b_{h}\right) \in S_{h}$ (note that it may be $S_{h}=S_{h^{\prime}}$ for $\left.h \neq h^{\prime}\right)$. Since each $S_{h}$ is connected, for every $h$ we can find a smooth embedding $\tau_{h}: I_{h} \rightarrow S_{h}$ with the property that $\tau_{h}\left(a_{h}\right)=\gamma\left(a_{h}\right)$ and $\tau_{h}\left(b_{h}\right)=\gamma\left(b_{h}\right)$. Furthermore, this can be achieved under the additional condition that $\tau_{h}\left(I_{h}\right) \cap \tau_{h^{\prime}}\left(I_{h^{\prime}}\right)=\emptyset$ for every $h \neq h^{\prime}$. We can then define a piecewise smooth embedding $\tilde{\gamma}$ of $\mathbb{S}^{1}$ into $\mathbb{R}^{n+1} \backslash \partial \Gamma_{0}$ such that $\left.\tilde{\gamma}\right|_{I_{h}}:=\left.\tau_{h}\right|_{I_{h}}$ for every $h$, and $\tilde{\gamma}=\gamma$ on the open set $\mathbb{S}^{1} \backslash \bigcup_{h} I_{h}$. We have $[\tilde{\gamma}]=[\gamma]$ in $\pi_{1}\left(\mathbb{R}^{n+1} \backslash \partial \Gamma_{0}\right)$. We can then construct a smooth embedding $\hat{\gamma}: \mathbb{S}^{1} \rightarrow \mathbb{R}^{n+1} \backslash \partial \Gamma_{0}$ such that $[\hat{\gamma}]=[\gamma]$ in $\pi_{1}\left(\mathbb{R}^{n+1} \backslash \partial \Gamma_{0}\right)$, and with $\hat{\gamma} \subset \mathbb{R}^{n+1} \backslash \partial U$. Since $n+1 \geq 3$ this contradicts the assumption that $[\gamma] \neq 0$ and completes the proof if $\gamma$ and $\partial U$ intersect transversally.

Finally, we remove the transversality assumption. Let $\delta=\delta(\partial U)>0$ be such that the tubular neighborhood $(\partial U)_{2 \delta}$ has a well-defined smooth nearest point projection $\Pi$, and consider, for $|s|<\delta$, the open sets $U_{s}$ having boundary $\partial U_{s}=\left\{x-s v_{U}(x): x \in \partial U\right\}$, where $v_{U}$ is the exterior normal unit vector field to $\partial U$. Since $\gamma$ is smooth, by Sard's theorem $\gamma$ intersects $\partial U_{s}$ transversally for a.e. $|s|<\delta$. Fix such an $s \in(0, \delta)$, and let $\Phi_{s}: \mathbb{R}^{n+1} \rightarrow \mathbb{R}^{n+1}$ be the smooth diffeomorphism of $\mathbb{R}^{n+1}$ defined by

$$
\Phi_{s}(x):=x+\varphi_{s}\left(\rho_{U}(x)\right) v_{U}(\Pi(x)),
$$

where

$$
\rho_{U}(x):= \begin{cases}|x-\Pi(x)| & \text { if } x \in(\partial U)_{2 \delta} \cap U \\ -|x-\Pi(x)| & \text { if } x \in(\partial U)_{2 \delta} \backslash U\end{cases}
$$




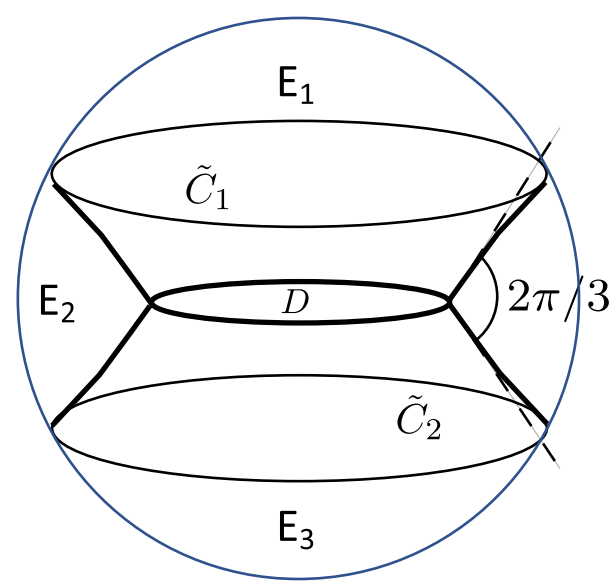

Fig. 1 The singular limit varifold detailed in Example 7.5

is the signed distance function from $\partial U$, and $\varphi_{s}=\varphi_{s}(t)$ is a smooth function such that

$$
\varphi_{s}(t)=0 \text { for all }|t| \geq 2 s, \quad \text { and } \quad \varphi_{s}(s)=s .
$$

In particular, $\Phi_{s}$ maps $\partial U_{s}$ diffeomorphically onto $\partial U$, and furthermore

$$
\Phi_{s} \rightarrow \text { id } \quad \text { uniformly on } \mathbb{R}^{n+1} \text { as } s \rightarrow 0+.
$$

Since $\gamma$ intersects $\partial U_{s}$ transversally, the curve $\Phi_{s} \circ \gamma$ intersects $\partial U$ transversally. Furthermore, since $\gamma$ and $\partial \Gamma_{0}$ are two compact sets with empty intersection, (7.13) implies that if we choose $s$ sufficiently small then also $\left(\Phi_{s} \circ \gamma\right) \cap \partial \Gamma_{0}=\emptyset$. Since $\left[\Phi_{s} \circ \gamma\right]=[\gamma] \neq 0$ in $\pi_{1}\left(\mathbb{R}^{n+1} \backslash \partial \Gamma_{0}\right)$, the first part of the proof guarantees that for every $t \in[0, \infty]$ we have $\Gamma(t) \cap\left(\Phi_{s} \circ \gamma\right) \neq \emptyset$. For every $t$ we then have points $z_{s}(t) \in \Gamma(t) \cap \Phi_{s} \circ \gamma$. Along a sequence $s_{h} \rightarrow 0+$, then, by compactness, (7.13), and the fact that each set $\Gamma(t)$ is closed, we have that the points $z_{s_{h}}(t)$ converge to a point $z_{0}(t) \in \Gamma(t) \cap \gamma$. The proof is now complete.

Example 7.5 Suppose that $U=U_{1}(0) \subset \mathbb{R}^{3}$, and $\partial \Gamma_{0}$ is the union of two parallel circles contained in $\mathbb{S}^{2}=\partial U$ at distance $2 h$ from one another, with $h \in(0,1)$. Then, $\partial U \backslash \partial \Gamma_{0}$ consists of the union of three connected components $S_{u} \cup S_{l} \cup S_{d}$ (here $u, l, d$ stand for up, lateral, and down, respectively). If $h$ is suitably small, then there are two smooth minimal catenoidal surfaces $C_{1} \subset U$ and $C_{2} \subset U$, one stable and the other unstable, satisfying $\operatorname{clos}\left(C_{j}\right) \backslash U=\partial \Gamma_{0}$. Nonetheless if the initial partition $\left\{E_{0, i}\right\}_{i}$ satisfies $(\diamond)$, then, as a consequence of Proposition 7.4, both $C_{1}$ and $C_{2}$ are not admissible limits of Brakke flow as in Corollary 2.4, since there exists a smooth and homotopically non-trivial embedding $\gamma: \mathbb{S}^{1} \rightarrow \mathbb{R}^{3} \backslash \partial \Gamma_{0}$ having empty intersection with each of them. For instances, if $N=3$ and the initial partition is such that $S_{u} \subset \operatorname{clos} E_{0,1}, S_{l} \subset \operatorname{clos} E_{0,2}$, and $S_{d} \subset \operatorname{clos} E_{0,3}$, then the corresponding Brakke flows will converge, instead, to a singular minimal surface $\Gamma$ in $U$ consisting of the union $\Gamma=\tilde{C}_{1} \cup \tilde{C}_{2} \cup D$, where $\tilde{C}_{j}$ are pieces of catenoids, and $D$ is a disc contained in the plane $\{z=0\}$, which join together forming $120^{\circ}$ angles along the "free boundary" circle $\Sigma=\partial D$; see Fig. 1 .

We will conclude the section with three remarks containing some interesting possible future research directions. 

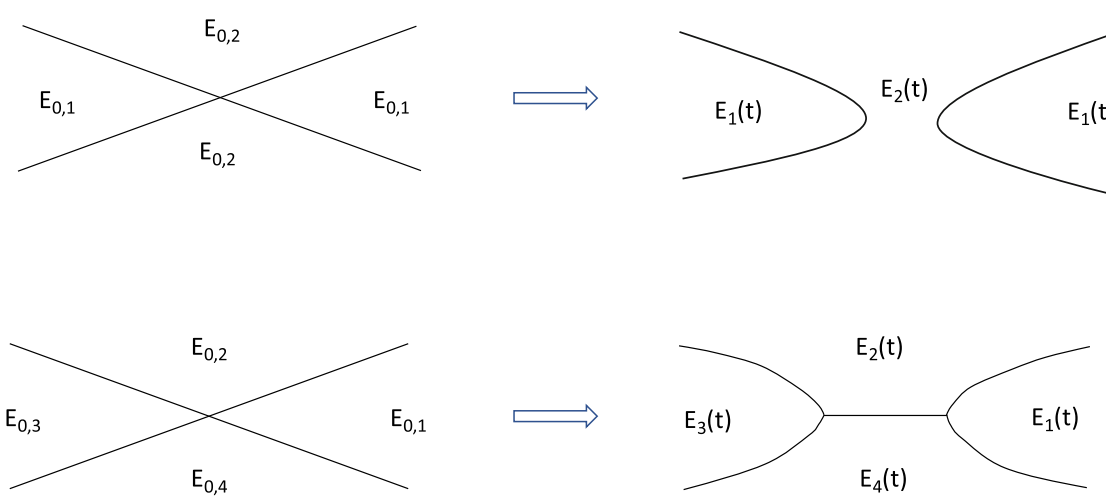

Fig. 2 Non-uniqueness without loss of mesure when $N=2$ (a) or $N=4$ (b)

Remark 7.6 First, we stress that the requirements on $\partial \Gamma_{0}$ are rather flexible, above all in terms of regularity. It would be interesting to characterize, for a given strictly convex domain $U \subset \mathbb{R}^{n+1}$, all its admissible boundaries, namely all subsets $\Sigma \subset \partial U$ such that there are $N \geq 2$ and $\mathcal{E}_{0}, \Gamma_{0}$ as in Assumption 1.1 such that $\Sigma=\partial \Gamma_{0}$. A first observation is that admissible boundaries do not need to be countably $(n-1)$-rectifiable, or to have finite $(n-1)$ dimensional Hausdorff measure: for example, it is not difficult to construct an admissible $\Sigma \subset \partial U_{1}(0)$ in $\mathbb{R}^{2}$ with $\mathcal{H}^{1}(\Sigma)>0$, essentially a "fat" Cantor set in $\mathbb{S}^{1}$. The assumption $(A 4)$ requires any admissible boundary to have empty interior. It is unclear whether this condition is also sufficient for a subset $\Sigma$ to be admissible.

Remark 7.7 Let us explicitly observe that, even in the case when $\Gamma_{0}$ (or more precisely $\left.V_{0}:=\operatorname{var}\left(\Gamma_{0}, 1\right)\right)$ is stationary, it is false in general that $V_{t}=V_{0}$ for $t>0$. In other words, the approximation scheme which produces the Brakke flow $V_{t}$ may move the initial datum $V_{0}$ even when the latter is stationary. A simple example is a set consisting of two line segments with a crossing, for which multiple non-trivial solutions (depending on the choice of the initial partition) are possible; see Fig. 2. In fact, one can prove that such one-dimensional configuration cannot stay time-independent with respect to the Brakke flow constructed in the present paper: [21, Theorem 2.2], indeed, shows that one-dimensional Brakke flows obtained in the present paper and in [20] necessarily satisfy a specific angle condition at junctions for a.e. time, with the only admissible angles being 0,60 , or 120 degrees. Thus, depending on the initial labeling of domains, one of the two evolutions depicted in Fig. 2 has to occur instantly.

If $\Gamma_{0}$ is a smooth minimal surface with smooth boundary $\partial \Gamma_{0}$, the uniqueness theorem for classical MCF should allow $\Gamma_{t} \equiv \Gamma_{0}$ as the unique solution, even if the latter is unstable (i.e. the second variation is negative for some direction). In other words, in the smooth case we expect that there is no other Brakke flow starting from $\Gamma_{0}$ other than the time-independent solution (notice, in passing, that both the area-reducing Lipschitz deformation step and the motion by smoothed mean curvature step in our time-discrete approximation of Brakke flow trivialize in this case - at least locally -, because smooth minimal surfaces are already locally area minimizing at suitably small scales around each point).

On the other hand, in [36] we show that time-dependent solutions may arise even from the existence, on $\Gamma_{0}$, of singular points at which $V_{0}$ has a flat tangent cone, that is a tangent cone which is a plane $T$ with multiplicity $Q \geq 2$. It would be interesting to characterize the regularity properties of those stationary $\Gamma_{0}$ with $E_{0,1}, \ldots, E_{0, N}$ satisfying Assumption 1.1 
and $\mathcal{H}^{n}\left(\Gamma_{0} \backslash \cup_{i=1}^{N} \partial^{*} E_{0, i}\right)=0$ which do not allow any non-trivial Brakke flows (dynamically stable stationary varifolds, in the terminology introduced in [36]). We expect that such a $\Gamma_{0}$ should have some local measure minimizing properties.

Remark 7.8 Let $V,\left\{E_{i}\right\}_{i=1}^{N}$ and $\Gamma$ be as in Corollary 2.4 obtained as $t_{k} \rightarrow \infty$ along a Brakke flow. Since $V$ is integral and stationary, $V=\operatorname{var}(\Gamma, \theta)$ for some $\mathcal{H}^{n}$-measurable function $\theta: \Gamma \rightarrow \mathbb{N}$. One can check that $\Gamma$ and $\left\{E_{i}\right\}_{i=1}^{N}$ (after removing empty $E_{i}$ 's if necessary) again satisfy the Assumption 1.1, thus we may apply Theorem 2.2 and obtain another Brakke flow with the same fixed boundary. Note that if we have $\|V\|(\{x: \theta(x) \geq 2\})>0$, then $\operatorname{var}(\Gamma, 1)$ may not be stationary, and the Brakke flow starting from non-stationary $\operatorname{var}(\Gamma, 1)$ is genuinely time-dependent. We then obtain another stationary varifold as $t \rightarrow \infty$ by Corollary 2.4. It is likely that, after a finite number of iterations, this process produces a unit density stationary varifold which does not move anymore. The other possibility is also interesting, in that we would have infinitely many different integral stationary varifolds with the same boundary condition, each having strictly smaller $\mathcal{H}^{n}$ measure than the previous one.

Acknowledgements The work of S.S. was supported by the NSF grants DMS-1565354, DMS-RTG-1840314 and DMS-FRG-1854344. Y.T. was partially supported by JSPS Grant-in-aid for scientific research 18H03670, $19 \mathrm{H} 00639$ and $17 \mathrm{H} 01092$

Open Access This article is licensed under a Creative Commons Attribution 4.0 International License, which permits use, sharing, adaptation, distribution and reproduction in any medium or format, as long as you give appropriate credit to the original author(s) and the source, provide a link to the Creative Commons licence, and indicate if changes were made. The images or other third party material in this article are included in the article's Creative Commons licence, unless indicated otherwise in a credit line to the material. If material is not included in the article's Creative Commons licence and your intended use is not permitted by statutory regulation or exceeds the permitted use, you will need to obtain permission directly from the copyright holder. To view a copy of this licence, visit http://creativecommons.org/licenses/by/4.0/.

\section{References}

1. Allard, W.K.: On the first variation of a varifold. Ann. Math. 2(95), 417-491 (1972)

2. Brakke, K.A.: The Motion of a Surface by Its Mean Curvaturevolume, volume 20 of Mathematical Notes. Princeton University Press, Princeton (1978)

3. Brakke, K.A.: Soap films and covering spaces. J. Geom. Anal. 5(4), 445-514 (1995)

4. Chen, Y.G., Giga, Y., Goto, S.: Uniqueness and existence of viscosity solutions of generalized mean curvature flow equations. J. Differ. Geom. 33(3), 749-786 (1991)

5. David, G.: Regularity of minimal and almost minimal sets and cones: J. Taylor's theorem for beginners. In: Analysis and Geometry of Metric Measure Spaces, volume 56 of CRM Proceedings. Lecture Notes, pp. 67-117. American Mathematical Society, Providence (2013)

6. David, G.: Should we solve Plateau's problem again? In: Advances in Analysis: the Legacy of Elias M. Stein, volume 50 of Princeton Math. Ser., pp. 108-145. Princeton University Press, Princeton (2014)

7. De Lellis, C., Ghiraldin, F., Maggi, F.: A direct approach to Plateau's problem. J. Eur. Math. Soc. (JEMS) 19(8), 2219-2240 (2017)

8. De Lellis, C., Hirsch, J., Marchese, A., Stuvard, S.: Area-minimizing currents mod 2Q: linear regularity theory. Commun. Pure Appl. Math. https://doi.org/10.1002/cpa.21964 (2020)

9. De Lellis, C., Hirsch, J., Marchese, A., Stuvard, S.: Regularity of area minimizing currents mod p. Geom. Funct. Anal. 30(5), 1224-1336 (2020)

10. Evans, L.C., Spruck, J.: Motion of level sets by mean curvature. I. J. Differ. Geom. 33(3), 635-681 (1991)

11. Federer, H.: Geometric measure theory. Die Grundlehren der mathematischen Wissenschaften, Band 153. Springer, New York (1969)

12. Federer, H., Fleming, W.H.: Normal and integral currents. Ann. Math. 2(72), 458-520 (1960)

13. Harrison, J.: Soap film solutions to Plateau's problem. J. Geom. Anal. 24(1), 271-297 (2014)

14. Harrison, J., Pugh, H.: Existence and soap film regularity of solutions to Plateau's problem. Adv. Calc. Var. 9(4), 357-394 (2016) 
15. Harrison, J., Pugh, H.: Plateau's problem. In: Open Problems in Mathematics, pp. 273-302. Springer, Cham (2016)

16. Huisken, G.: Nonparametric mean curvature evolution with boundary conditions. J. Differ. Equ. 77(2), 369-378 (1989)

17. Ilmanen, T.: Elliptic regularization and partial regularity for motion by mean curvature. Mem. Am. Math. Soc. 108 $(520), x+90(1994)$

18. Ilmanen, T., Sternberg, P., Ziemer, W.P.: Equilibrium solutions to generalized motion by mean curvature. J. Geom. Anal. 8(5), 845-858 (1998). Dedicated to the memory of Fred Almgren

19. Kasai, K., Tonegawa, Y.: A general regularity theory for weak mean curvature flow. Calc. Var. Partial Differ. Equ. 50(1-2), 1-68 (2014)

20. Kim, L., Tonegawa, Y.: On the mean curvature flow of grain boundaries. Ann. Inst. Fourier (Grenoble) 67(1), 43-142 (2017)

21. Kim, L., Tonegawa, Y.: Existence and regularity theorems of one-dimensional Brakke flows. Interfaces Free Bound. 22, 505-550 (2020)

22. King, D., Maggi, F., Stuvard, S.: Collapsing and the convex hull property in a soap film capillarity model. Ann. Inst. H. Poincaré Anal. Non Linéaire (2021)

23. King, D., Maggi, F., Stuvard, S.: Plateau's problem as a singular limit of capillarity problems. Commun. Pure Appl. Math. https://doi.org/10.1002/cpa.21951 (2020)

24. King, D., Maggi, F., Stuvard, S.: Smoothness of collapsed regions in a capillarity model for soap films. arXiv preprint arXiv:2007.14868 (2020)

25. Lieberman, G.M.: The first initial-boundary value problem for quasilinear second order parabolic equations. Ann. Scuola Norm. Sup. Pisa Cl. Sci. (4) 13(3), 347-387 (1986)

26. Maggi, F.: Sets of Finite Perimeter and Geometric Variational Problems, volume 135 of Cambridge Studies in Advanced Mathematics. Cambridge University Press, Cambridge (2012)

27. Maggi, F., Scardicchio, A., Stuvard, S.: Soap films with gravity and almost-minimal surfaces. Discrete Cont. Dyn. Syst. 39(12), 6877-6912 (2019)

28. Magni, A., Mantegazza, C., Novaga, M.: Motion by curvature of planar networks, II. Ann. Sc. Norm. Super. Pisa Cl. Sci. (5) 15, 117-144 (2016)

29. Mantegazza, C., Novaga, M., Pluda, A., Schulze, F.: Evolution of networks with multiple junctions. arXiv preprint arXiv:1611.08254 (2016)

30. Mantegazza, C., Novaga, M., Tortorelli, V.M.: Motion by curvature of planar networks. Ann. Sc. Norm. Super. Pisa Cl. Sci. (5) 3(2), 235-324 (2004)

31. Priwitzer, B.: Mean curvature flow with Dirichlet boundary conditions in Riemannian manifolds with symmetries. Ann. Global Anal. Geom. 23(2), 157-171 (2003)

32. Schulze, F., White, B.: A local regularity theorem for mean curvature flow with triple edges. J. Reine Angew. Math. 758, 281-305 (2020)

33. Simon, L.: Lectures on geometric measure theory, volume 3 of Proceedings of the Centre for Mathematical Analysis, Australian National University. Australian National University, Centre for Mathematical Analysis, Canberra (1983)

34. Spruck, J.: Interior gradient estimates and existence theorems for constant mean curvature graphs in $M^{n} \times \mathbf{R}$. Pure Appl. Math. Q., 3(3, Special Issue: In honor of Leon Simon. Part 2):785-800 (2007)

35. Sternberg, P., Ziemer, W.P.: Generalized motion by curvature with a Dirichlet condition. J. Differ. Equ. 114(2), 580-600 (1994)

36. Stuvard, S., Tonegawa, Y.: Dynamical instability of minimal surfaces at flat singular points. arXiv preprint arXiv:2008.13728 (2020)

37. Tonegawa, Y.: A second derivative Hölder estimate for weak mean curvature flow. Adv. Calc. Var. 7(1), 91-138 (2014)

38. Tonegawa, Y.: Brakke's Mean Curvature Flow: An Introduction. SpringerBriefs in Mathematics. Springer, Singapore (2019)

39. White, B.: Mean curvature flow with boundary. arXiv preprint arXiv:1901.03008 (2019)

Publisher's Note Springer Nature remains neutral with regard to jurisdictional claims in published maps and institutional affiliations. 